

\section{Cell-Free Massive MIMO: Scalability, Signal Processing and Power Control}

Giovanni Interdonato

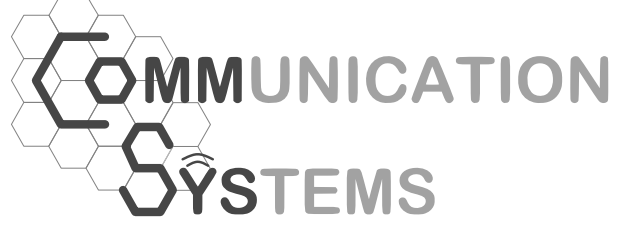

Division of Communication Systems

Department of Electrical Engineering (ISY) Linköping University, 58183 Linköping, Sweden www.commsys.isy.liu.se

Linköping 2020 
This is a Swedish Doctor of Philosophy thesis.

The Doctor of Philosophy degree comprises 240 ECTS credits of postgraduate studies.

Cover: Illustration of the Ericsson Radio Stripes concept, co-invented by the author of this thesis. The Radio Stripes constitute a way to implement a cell-free massive MIMO system. They aim at enabling invisible, low-cost deployment of large number of distributed access points in the vicinity of the users. This is achieved by serial integration of several transmitting and receiving components into a cable (or stripe) which also provides fronthaul communication and power supply. Visit https://www.ericsson.com/en/blog/2019/2/radio-stripes for further details.

\section{Cell-Free Massive MIMO: Scalability, Signal Processing and Power Control}

(c) 2020 Giovanni Interdonato, unless otherwise stated.

ISBN 978-91-7929-808-1

ISSN 0345-7524

URL http://urn.kb.se/resolve?urn=urn:nbn:se:liu:diva-167218

Printed in Sweden by LiU-Tryck, Linköping 2020 
To my one and only love, Silvia

"Nobody said it was easy No one ever said it would be so hard I'm going back to the start"

C. Martin 



\section{Abstract}

The fifth generation of mobile communication systems $(5 \mathrm{G})$ is nowadays a reality. $5 \mathrm{G}$ networks are been deployed all over the world, and the first 5G-capable devices (e.g., smartphones, tablets, wearable, etc.) are already commercially available. 5G systems provide unprecedented levels of connectivity and quality of service (QoS) to cope with the incessant growth in the number of connected devices and the huge increase in data-rate demand.

Massive MIMO (multiple-input multiple-output) technology plays a key role in $5 \mathrm{G}$ systems. The underlying principle of this technology is the use of a large number of co-located antennas at the base station, which coherently transmit/receive signals to/from multiple users. This signal co-processing at multiple antennas leads to manifold benefits: array gain, spatial diversity and spatial user multiplexing. These elements enable to meet the QoS requirements established for the $5 \mathrm{G}$ systems. The major bottleneck of massive MIMO systems as well as of any cellular network is the inter-cell interference, which affects significantly the cell-edge users, whose performance is already degraded by the path attenuation. To overcome these limitations and provide uniformly excellent service to all the users we need a more radical approach: we need to challenge the cellular paradigm.

In this regard, cell-free massive MIMO constitutes the paradigm shift. In the cell-free paradigm, it is not the base station surrounded by the users, but rather it is each user being surrounded by smaller, simpler, serving base stations referred to as access points (APs). In such a system, each user experiences being in the cell-center, and it does not experience any cell boundaries. Hence, the terminology cell-free. As a result, users are not affected by inter-cell interference, and the path attenuation is significantly reduced due to the presence of many APs in their proximity. This leads to impressive performance.

Although appealing from the performance viewpoint, the designing and implementation of such a distributed massive MIMO system is a challenging task, and it is the object of this thesis. More specifically, in this thesis we study:

Paper A) The large potential of this promising technology in realistic indoor/outdoor 
scenarios while also addressing practical deployment issues, such as clock synchronization among APs, and cost-efficient implementations. We provide an extensive description of a cell-free massive MIMO system, emphasizing strengths and weaknesses, and pointing out differences and similarities with existing distributed multiple antenna systems, such as Coordinated MultiPoint (CoMP).

Paper B) How to preserve the scalability of the system, by proposing a solution related to data processing, network topology and power control. We consider a realistic scenario where multiple central processing units serve disjoint subsets of APs, and compare the spectral efficiency provided by the proposed scalable framework with the canonical cell-free massive MIMO and CoMP.

Paper C) How to improve the spectral efficiency (SE) in the downlink (DL), by devising two distributed precoding schemes, referred to as local partial zero-forcing (ZF) and local protective partial ZF, that provide an adaptable trade-off between interference cancelation and boosting of the desired signal, with no additional front-haul overhead, and that are implementable by APs with very few antennas. We derive closed-form expressions for the achievable SE under the assumption of independent Rayleigh fading channel, channel estimation error and pilot contamination. These closed-form expressions are then used to devise optimal max-min fairness power control.

Paper D) How to further improve the SE by letting the user estimate the DL channel from DL pilots, instead of relying solely on the knowledge of the channel statistics. We derive an approximate closed-form expression of the DL SE for conjugate beamforming $(\mathrm{CB})$, and assuming independent Rayleigh fading. This expression accounts for beamformed DL pilots, estimation errors and pilot contamination at both the AP and the user side. We devise a sequential convex approximation algorithm to globally solve the max-min fairness power control optimization problem, and a greedy algorithm for uplink (UL) and DL pilot assignment. The latter consists in jointly selecting the UL and DL pilot pair, for each user, that maximizes the smallest SE in the network.

Paper E) A precoding scheme that is more suitable when only the channel statistics are available at the users, referred to as enhanced normalized CB. It consists in normalizing the precoding vector by its squared norm in order to reduce the fluctuations of the effective channel seen at the user, and thereby to boost the channel hardening. The performance achieved by this scheme is compared with the CB scheme with DL training (described in Paper D).

Paper F) A maximum-likelihood-based method to estimate the channel statistics in the UL, along with an accompanying pilot transmission scheme, that is particularly useful in line-of-sight operation and in scenarios with resource constraints. 
Pilots are structurally phase-rotated over different coherence blocks to create an effective statistical distribution of the received pilot signal that can be efficiently exploited by the AP when performing the proposed estimation method.

The overall conclusion is that cell-free massive MIMO is not a utopia, and a practical, distributed, scalable, high-performance system can be implemented. Today it represents a hot research topic, but tomorrow it might represent a key enabler for beyond-5G technology, as massive MIMO has been for $5 \mathrm{G}$. 



\section{Premessa}

La quinta generazione dei sistemi radiomobili cellulari $(5 \mathrm{G})$ è oggi una realtà. Le reti 5G si stanno diffondendo in tutto il mondo e i dispositivi 5G (ad esempio smartphones, tablets, indossabili, ecc.) sono già disponibili sul mercato. I sistemi $5 \mathrm{G}$ garantiscono livelli di connettività e di qualità di servizio senza precedenti, per fronteggiare l'incessante crescita del numero di dispositivi connessi alla rete e della domanda di dati ad alta velocità.

La tecnologia Massive MIMO (multiple-input multiple-output) riveste un ruolo fondamentale nei sistemi 5G. Il principio alla base di questa tecnologia è l'impiego di un elevato numero di antenne collocate nella base station (stazione radio base) le quali trasmettono/ricevono segnali, in maniere coerente, a/da più terminali utente. Questo co-processamento del segnale da parte di più antenne apporta molteplici benefici: guadagno di array, diversità spaziale e multiplazione degli utenti nel dominio spaziale. Questi elementi consentono di raggiungere i requisiti di servizio stabiliti per i sistemi 5G. Tuttavia, il limite principale dei sistemi massive MIMO, così come di ogni rete cellulare, è rappresentato dalla interferenza intercella (ovvero l'interferenza tra aree di copertura gestite da diverse base stations), la quale riduce in modo significativo le performance degli utenti a bordo cella, già degradate dalle attenuazioni del segnale dovute alla considerevole distanza dalla base station. Per superare queste limitazioni e fornire un qualità del servizio uniformemente eccellente a tutti gli utenti, è necessario un approccio più radicale $\mathrm{e}$ guardare oltre il classico paradigma cellulare che caratterizza le attuali architetture di rete.

A tal proposito, cell-free massive MIMO (massive MIMO senza celle) costituisce un cambio di paradigma: ogni utente è circondato e servito contemporaneamente da numerose, semplici e di dimensioni ridotte base stations, denominate access points (punti di accesso alla rete). Gli access points cooperano per servire tutti gli utenti nella loro area di copertura congiunta, eliminando l'interferenza intercella e il concetto stesso di cella. Non risentendo più dell'effetto "bordo-cella", gli utenti possono usufruire di qualità di servizio e velocità dati eccellenti. Sebbene attraente dal punto di vista delle performance, l'implementazione di un tale sis- 
tema distribuito è una operazione impegnativa ed è oggetto di questa tesi. Più specificatamente, questa tesi di dottorato tratta:

Articolo A) L'enorme potenziale di questa promettente tecnologia in scenari realistici sia indoor che outdoor, proponendo anche delle soluzioni di implementazione flessibili ed a basso costo.

Articolo B) Come preservare la scalabilità del sistema, proponendo soluzioni distribuite riguardanti il processamento e la condivisione dei dati, l'architettura di rete e l'allocazione di potenza, ovvero come ottimizzare i livelli di potenza trasmessa dagli access points per ridurre l'interferenza tra utenti e migliorare le performance.

Articolo C) Come migliorare l'efficienza spettrale in downlink (da access point verso utente) proponendo due schemi di pre-codifica dei dati di trasmissione, denominati local partial zero-forcing (ZF) e local protective partial ZF, che forniscono un perfetto compromesso tra cancellazione dell'interferenza tra utenti ed amplificazione del segnale desiderato.

Articolo D) Come migliorare l'efficienza spettrale in downlink permettendo al terminale utente di stimare le informazioni sulle condizioni istantanee del canale da sequenze pilota, piuttosto che basarsi su informazioni statistiche ed a lungo termine, come convenzionalmente previsto.

Articolo E) In alternativa alla soluzione precedente, uno schema di pre-codifica che è più adatto al caso in cui gli utenti hanno a disposizione esclusivamente informazioni statistiche sul canale per poter effettuare la decodifica dei dati.

Articolo F) Un metodo per permettere agli access points di stimare, in maniera rapida, le condizioni di canale su base statistica, favorito da uno schema di trasmissione delle sequenze pilota basato su rotazione di fase.

Realizzare un sistema cell-free massive MIMO pratico, distribuito, scalabile e performante non è una utopia. Oggi questo concept rappresenta un argomento di ricerca interessante, attraente e stimolante ma in futuro potrebbe costituire un fattore chiave per le tecnologie post-5G, proprio come massive MIMO lo è stato per il 5G. 


\section{Populärvetenskaplig Sammanfattning}

Den femte generationens mobilkommunikationssystem (5G) är numera en verklighet. 5G-nätverk är utplacerade på ett flertal platser världen över och de första 5G-kapabla terminalerna (såsom smarta telefoner, surfplattor, kroppsburna apparater, etc.) är redan kommersiellt tillgängliga. 5G-systemen kan tillhandahålla tidigare oöverträffade nivåer av uppkoppling och servicekvalitet och är designade för en fortsatt oavbruten tillväxt i antalet uppkopplade apparater och ökande datataktskrav.

Massiv MIMO-teknologi (eng: multiple-input multiple-output) spelar en nyckelroll i dagens 5G-system. Principen bakom denna teknik är användningen av ett stort antal samlokaliserade antenner vid basstationen, där alla antennerna sänder och tar emot signaler faskoherent till och från flera användare. Gemensam signalbehandling av många antennsignaler ger ett flertal fördelar, såsom hög riktverkan via lobformning, vilket leder till högre datatakter samt möjliggör att flera användare utnyttjar samma radioresurser via rumslig användarmultiplexering. Eftersom en signal kan gå genom flera olika, möjligen oberoende kanaler, så utsätts den för flera olika förändringar samtidigt. Denna mångfald ökar kvaliteten på signalen vid mottagaren och förbättrar radiolänkens robusthet och tillförlitlighet. Detta gör det möjligt att uppfylla de höga kraven på servicekvalitet som fastställts för 5G-systemen.

Den största begränsningen för massiva MIMO-system såväl som för alla cellulära mobilnätverk, är störningar från andra celler som påverkar användare på cellkanten väsentligt, vars prestanda redan begränsas av sträckdämpningen på radiokanalen. För att övervinna dessa begränsningar och för att kunna tillhandahålla samma utmärkta servicekvalitet till alla användare behöver vi ett mer radikalt angreppssätt: vi måste utmana cellparadigmet.

I detta avseende utgör cellfri massiv-MIMO teknik ett paradigmskifte. I cellfri massive-MIMO är utgångspunkten inte att basstationen är omgiven av användare 
som den betjänar, utan snarare att varje användare omges av basstationer som de betjänas av. Dessa basstationer, ofta mindre och enklare, kallas accesspunkter (AP). I ett sådant system upplever varje användare att den befinner sig i centrum av systemet och ingen användare upplever några cellgränser. Därav terminologin cellfri. Som ett resultat av detta påverkas inte användarna av inter-cellstörningar och sträckdämpningen reduceras kraftigt på grund av närvaron av många accesspunkter i varje användares närhet. Detta leder till imponerande prestanda.

Även om det är tilltalande ur ett prestandaperspektiv så är utformningen och implementeringen av ett sådant distribuerat massivt MIMO-system en utmanande uppgift, och det är syftet med denna avhandling att studera detta. Mer specifikt studerar vi i denna avhandling: A) den mycket stora potentialen med denna teknik i realistiska inomhus- såväl som utomhusscenarier, samt hur man hanterar praktiska implementeringsproblem, såsom klocksynkronisering bland accesspunkter och kostnadseffektiva implementeringar; B) hur man ska uppnå skalbarhet i systemet genom att föreslå lösningar relaterade till databehandling, nätverkstopologi och effektkontroll; C) hur man ökar datahastigheten i nedlänken med hjälp av två nyutvecklade distribuerade överföringsmetoder som tillhandahåller en avvägning mellan störningsundertryckning och förstärkning av önskade signaler, utan att öka mängden intern signalering till de distribuerade accesspunkterna, och som kan implementeras i accesspunkter med mycket få antenner; D) hur man kan förbättra prestandan ytterligare genom att låta användaren estimera nedlänkskanalen med hjälp av nedlänkspiloter, istället för att bara förlita sig på kunskap om kanalstatistik; E) en överföringsmetod för nedlänk som är mer lämpligt när endast kanalstatistiken är tillgänglig för användarna. Prestandan som uppnås genom detta schema jämförs med en utökad variant av den nedlänk-pilotbaserade metoden (beskrivet i föregående punkt); F) en metod för att uppskatta kanalstatistiken i upplänken, samt en åtföljande pilotsändningsmetod, som är särskilt användbart vid direktvägsutbredning (line-of-sight) och i scenarier med resursbegränsningar.

Den övergripande slutsatsen är att cellfri massiv MIMO inte är en utopi, och att ett distribuerat, skalbart, samt högpresterande system kan implementeras praktiskt. Idag representerar detta ett hett forskningsämne, men snart kan det visa sig vara en viktig möjliggörare för teknik bortom dagens system, på samma sätt som centraliserad massiv MIMO har varit för de nya 5G-systemen. 


\section{Contents}

$\begin{array}{ll}\text { Acknowledgements } & \text { xv }\end{array}$

List of Abbreviations $\quad$ xvii

1 Introduction and Motivation $\quad 1$

1.1 Contributions of the Thesis . . . . . . . . . . . . . . 7

1.2 Excluded Papers ..................... 12

2 Co-located MIMO Systems 13

2.1 Preliminaries ...................... 13

2.1.1 Channel Capacity and Outage Probability . . . . . . . 13

2.1 .2 SISO Channel . . . . . . . . . . . . . . . . . . . 14

2.2 Benefits of Using Multiple Antennas . . . . . . . . . . . . . . . 15

2.2.1 Beamforming Gain . . . . . . . . . . . . . . 15

2.2.2 Spatial Diversity Gain . . . . . . . . . . . . . 16

2.3 Point-to-Point MIMO . . . . . . . . . . . . . . . . . 17

2.3.1 Multiplexing Gain .............. . . . 17

2.3.2 Extra Spatial Diversity . . . . . . . . . . . . . 18

2.3.3 Practical Issues . . . . . . . . . . . . . . . . . 20

2.4 Multiuser MIMO . . . . . . . . . . . . . . . . . . . . . . 21

2.4.1 Sum Capacity . . . . . . . . . . . . . . 21

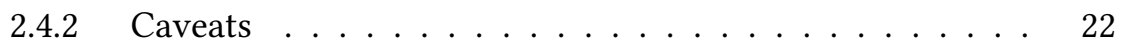

2.5 Massive MIMO . . . . . . . . . . . . . . . . . . 23

2.5.1 Key Ingredients . . . . . . . . . . . . . . . . . 23

2.5.2 Favorable Propagation .............. 25

2.5.3 Channel Hardening . . . . . . . . . . . . . . . . 28

2.5.4 TDD Operation ............... 29

3 Distributed MIMO Systems 31

3.1 Taxonomy ....................... 31 
3.1.1 Network MIMO . . . . . . . . . . . . . . . . . . . . . 31

3.1 .2 Cooperative Multi-cell MIMO . . . . . . . . . . . . 32

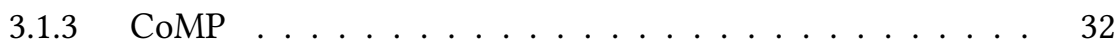

3.1 .4 C-RAN . . . . . . . . . . . . . . . 33

3.1.5 Related Concepts . . . . . . . . . . . . . . . . . . 33

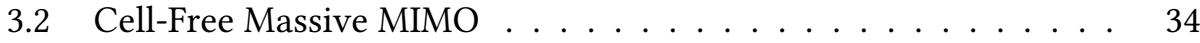

3.2 .1 Features . . . . . . . . . . . . . . . 34

3.2.2 Challenges and Key Enablers _. . . . . . . . . . . . 35

3.2.3 The Canonical Form: System Model and Performance . . 36

4 Scope and Related Works $\quad 43$

5 Conclusions and Future Directions 53

$\begin{array}{ll}\text { Bibliography } & 56\end{array}$

$\begin{array}{ll}\text { Included Papers } & 78\end{array}$

A Ubiquitous Cell-Free Massive MIMO Communications 79

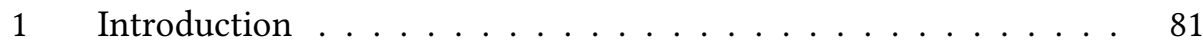

2 System Operation and Resource Allocation . . . . . . . . . . 84

2.1 Ubiquitous Cell-Free Massive MIMO: The Scalable Way to Implement CoMP-JT . . . . . . . . . . . . . . . . . . 84

2.2 TDD Protocol . . . . . . . . . . . . . . . 86

2.3 Uplink Pilot Assignment . . . . . . . . . . . . . . . . 88

$2.4 \quad$ Power Control . . . . . . . . . . . . . . . . . . 88

3 Practical Deployment Issues _ . . . . . . . . . . . . . 90

$3.1 \quad$ Radio Stripes System . . . . . . . . . . . . . . . . . . . . . 90

$3.2 \quad$ Front-haul and Back-haul Capacity . . . . . . . . . . . . 93

3.3 Synchronization . . . . . . . . . . . . . . 994

4 Performance of Ubiquitous Cell-Free Massive MIMO $\ldots \ldots \ldots$

4.1 Industrial Indoor Scenario . . . . . . . . . . . . . . . 95

4.2 Outdoor Piazza Scenario . . . . . . . . . . . . . . . . . 98

5 Conclusion: Where there's a will, there's a way . . . . . . . . 99

B Scalability Aspects of Cell-Free Massive MIMO 105

1 Introduction . . . . . . . . . . . . . . . . . . 107

2 The Scalability Problem . . . . . . . . . . . . . . . . . . . . . 108

$2.1 \quad$ Is Power Control Really Scalable? . . . . . . . . . . . . . . 109

$2.2 \quad$ User-centric vs Cell-centric Clustering . . . . . . . . . 110 
3 Proposed Solution . . . . . . . . . . . . . . . . . . . . . 111

$4 \quad$ Numerical Results . . . . . . . . . . . . . . . . . . . . . . . . . . . 113

$4.1 \quad$ Simulation Scenario . . . . . . . . . . . . . . . . 113

4.2 Spectral Efficiency Evaluation . . . . . . . . . . . . 115

4.3 Distributed Power Control Strategies . . . . . . . . . . . 117

5 Conclusion . . . . . . . . . . . . . . . . . . . . . . . 119

C Local Partial Zero-Forcing Precoding for Cell-Free Massive MIMO 123

1 Introduction . . . . . . . . . . . . . . . . . . . . 125

$1.1 \quad$ Motivation . . . . . . . . . . . . . . . 127

1.2 Contributions . . . . . . . . . . . . . . . . 128

2 System Model . . . . . . . . . . . . . . . . . . . . . . . . . 128

$2.1 \quad$ Uplink Training . . . . . . . . . . . . . . . . . . . . . 129

2.2 Downlink Data Transmission . . . . . . . . . . . . 130

3 Performance Analysis . . . . . . . . . . . . . . . . . . . . . . 131

3.1 Downlink Spectral Efficiency . . . . . . . . . . . . . 132

3.2 Maximum Ratio Transmission . . . . . . . . . . . . . . . 133

3.3 Full-pilot Zero-Forcing Precoding . . . . . . . . . . . . 133

$3.4 \quad$ Local Partial Zero-Forcing Precoding . . . . . . . . . . . 135

3.5 Local Protective Partial Zero-Forcing . . . . . . . . . . . . 139

$3.6 \quad$ Local Regularized Zero-Forcing . . . . . . . . . . . . . 140

4 Power Control . . . . . . . . . . . . . . . . . . . . . . . 141

$4.1 \quad$ Max-Min Fairness . . . . . . . . . . . . . . . . . . . 141

4.2 Distributed Heuristic Channel-Dependent Strategy . . . . 143

5 Front-hauling Costs and Computational Complexity . . . . . . . 143

6 Simulation Results . . . . . . . . . . . . . . . . . . . . . . . . 145

6.1 Simulation Scenario . . . . . . . . . . . . . . . 145

6.2 Performance Evaluation . . . . . . . . . . . . . . . 146

7 Conclusion . . . . . . . . . . . . . . . . . . 154

7.1 Proof of Corollary $1 \ldots \ldots \ldots \ldots$

7.2 Proof of Corollary $2 \ldots \ldots \ldots \ldots$

7.3 Proof of Corollary $3 \ldots \ldots \ldots \ldots$

D Downlink Training in Cell-Free Massive MIMO: A Blessing in Dis$\begin{array}{ll}\text { guise } & 165\end{array}$

1 Introduction . . . . . . . . . . . . . . . . 167

2 System Model . . . . . . . . . . . . . . . . . . . . . . 170

2.1 Uplink Training and Channel Estimation . . . . . . . . . 171

2.2 Downlink Data Transmission . . . . . . . . . . . 172

2.3 Downlink Training and Channel Estimation . . . . . . . 173 
3 Performance Analysis . . . . . . . . . . . . . . . . . . 176

$3.1 \quad$ Approximate Achievable Downlink Rate . . . . . . . . 176

3.2 Use-and-Forget Achievable Downlink Rate . . . . . . . . . 179

3.3 Downlink Pilots, or No Downlink Pilots, that is the Question 180

4 Resource Allocation . . . . . . . . . . . . . . . . . . . . 181

4.1 Max-Min Fairness Power Control . . . . . . . . . . . . 181

$4.2 \quad$ Pilot Sequence Assignment _... . . . . . . . . . . . 185

$5 \quad$ Numerical Results and Discussions _ . . . . . . . . . . . . . . 187

$5.1 \quad$ Simulation Scenario . . . . . . . . . . . . . . . . 187

5.2 Pilot Allocation . . . . . . . . . . . . . . . . . . . . 189

5.3 Performance of the SCA Algorithm for Max-Min Power Control . . . . . . . . . . . . . . . . . . . 191

5.4 Downlink Training Gain . . . . . . . . . . . . . . . . 192

5.5 Downlink Training Gain under Max-Min Power Control . 194

5.6 Downlink Training Gain in User-centric Massive MIMO Networks . . . . . . . . . . . . . . . . . . 196

6 Conclusion . . . . . . . . . . . . . . . . . . . . . . 197

6.1 Proof of Proposition $1 \ldots \ldots \ldots$

$6.2 \quad$ Proof of Proposition $2 \ldots \ldots \ldots$

E Enhanced Conjugate Beamforming for Cell-Free Massive MIMO 205

1 Introduction . . . . . . . . . . . . . . . . . 207

2 System Model . . . . . . . . . . . . . . . . . . . . . . . 210

2.1 Uplink Training . . . . . . . . . . . . . . . . . . . . 210

2.2 Downlink Data Transmission . . . . . . . . . . . . 211

3 Performance Analysis - No CSI at the User . . . . . . . . . . . . 212

3.1 Conjugate Beamforming . . . . . . . . . . . . . . 214

3.2 Normalized Conjugate Beamforming . . . . . . . . . . 214

3.3 Enhanced Normalized Conjugate Beamforming . . . . . . 215

4 Performance Analysis of Conjugate Beamforming with Downlink Training . . . . . . . . . . . . . . . 216

$5 \quad$ Max-Min Fairness Power Control . . . . . . . . . . . . . . . 218

$5.1 \quad$ Problem Formulation for NCB . . . . . . . . . . . . . . . 219

5.2 Problem Formulation for ECB . . . . . . . . . . . 220

5.3 Problem Formulation for CB with Downlink Training . . 221

6 Simulation Results . . . . . . . . . . . . . . . . . . . 222

6.1 Simulation Scenario and Settings _ . . . . . . . . . 222

6.2 Performance Evaluation . . . . . . . . . . . . . . . 224

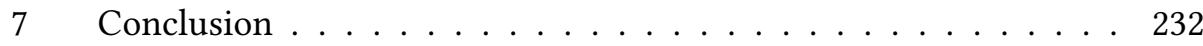


F Self-Learning Detector for the Cell-Free Massive MIMO Uplink 239

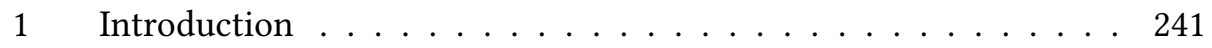

2 System Model . . . . . . . . . . . . . . . . . . . 243

$2.1 \quad$ Uplink Training . . . . . . . . . . . . . . . . . . . . . . . . . 243

2.2 Channel Estimation . . . . . . . . . . . . . . . . . . 244

3 Self-Learning Detector . . . . . . . . . . . . . . . . 245

$3.1 \quad$ Proposed Method . . . . . . . . . . . . . . . . . . 245

3.2 Analysis for the LoS Channel . . . . . . . . . . . . 247

4 Simulation Results . . . . . . . . . . . . . . . . . . . . . . 249

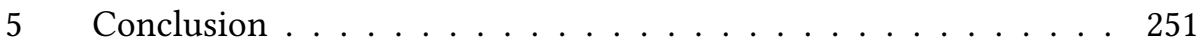

6 Annex: Agnostic Detector for Cell-Free Massive MIMO . . . . . . 252

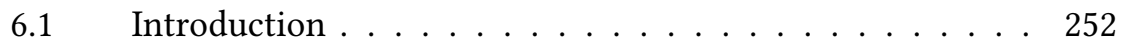

6.2 System Model . . . . . . . . . . . . . . . . . . . . 252

6.3 Agnostic Detector . . . . . . . . . . . . . . 255

6.4 Performance Evaluation . . . . . . . . . . . . . . 257

6.5 Conclusion: The User Ambiguity Issue . . . . . . . . . 259 



\section{Acknowledgements}

I owe my deepest gratitude to Prof. Erik G. Larsson and Dr. Gunnar Bark, the first who believed in me and gave me the opportunity to live this unforgettable experience.

I have been extremely lucky, proud and honored to be surrounded by brilliant colleagues and friends at the Division of Communication Systems of Linköping University and at the LINLAB group of Ericsson Research. I learned a lot from each and every one of you.

In addition to my supervisor Prof. Erik G. Larsson, special thanks go to my co-supervisor Dr. Hien Quoc Ngo and my mentors Dr. Pål Frenger and Prof. Emil Björnson for the continuous support and encouragement during my Ph.D. studies, teaching and research activities. Their motivation, enthusiasm and optimism, expertise and immense knowledge enabled me to grow not only as researcher and teacher but especially as a person. Without their guidance and persistent help this thesis would not have been possible.

In addition to my former manager at Ericsson Dr. Gunnar Bark, I express my gratitude to my current manager Dr. Nicklas Johansson. Their affection and care shown towards me, keen interest and involvement in my research and concern for my welfare have greatly motivated me. You have always been supportive of me and made me comfortable every single moment.

I am grateful to the " 5 Gwireless" project-H2020 Marie Skłodowska-Curie Innovative Training Networks-and Ericsson's Research Foundation for their financial support. My warm thanks to Prof. Marco Di Renzo for its hard work as coordinator of the 5Gwireless project, and its assistance during all the project activities. My heartiest thanks to my 5Gwireless colleagues for all the time we shared: we worked hard and played hard.

To all the true friends that I had the privilege to meet during this journey and in particular: Amin, Nastaran, Chien, Özgecan, Vedat, Meysam, Farnaz, Daniel, Ema, Marcus, Antonis and Alexis. We all have shared joys and difficulties, concerns and hopes. Without your closeness, pursuing a Ph.D. while living abroad and alone would have been an insurmountable obstacle. 
To Antonino: fate brought us together from Reggio Calabria to Ryd (amazing) to make us like brothers. Neither is expert in brotherhood, but as Benjamin Franklin said, "A brother may not be a friend, but a friend will always be a brother."

I have no words to express my love and gratitude to my lovely wife Silvia. We spent almost half of our lives together, I do not even remember how life was before you! Listing what you mean to me and what you have done for me would require a separate dissertation. You made me the man I am, being always by my side. You made this happen: you pushed me to apply for this Ph.D. position, you taught me to never give up and to keep pursuing my passions no matter what, even sacrificing yourself, ourselves. You gave me strength in difficult times. Never in my dreams did I think I would be lucky enough to be with a woman as you.

Last but not least, I am extremely grateful to my parents, Gaetano and Santina, for their love, prayers, support, care and encouraging words as well as Silvia's family, that is my complementary family: Antonello, Giovanna, Sarah who is the little sister I never had, and the "new entry" Antonio.

Giovanni Interdonato Linköping, August 2020 


\section{List of Abbreviations}

$5 G$

fifth generation of cellular network technology

AP

access point

AWGN

additive white Gaussian noise

CoMP

coordinated multipoint

CPU

central processing unit

CSI

channel state information

DL

downlink

DPC

dirty paper coding

ELAA

extremely large aperture array

eMBB

enhanced mobile broadband

FDD

frequency-division duplex

i.i.d. independent and identically distributed

IoT internet-of-things

LoS line-of-sight

LTE long-term evolution

MIMO multiple-input multiple-output

MISO multiple-input single-output

MMF max-min fairness

MMSE minimum mean-square error

mMTC massive machine-type communication

mmWave millimeter wave

MR

maximum ratio

NLoS

non line-of-sight

NR

New Radio 
QoS

$\mathrm{RF}$

SE

SIC

SIMO

SISO

SNR

SINR

TDD

UL

URLLC

ZF quality-of-service

radio frequency

spectral efficiency

successive interference cancellation

single-input multiple-output

single-input single-output

signal-to-noise ratio

signal-to-interference-plus-noise ratio

time-division duplex

uplink

ultra-reliable low-latency communication

zero forcing 


\section{Chapter 1}

\section{Introduction and Motivation}

Each generation of cellular network technology has made a step forward towards the ultimate mission of providing excellent communication service to anyone, anywhere, any time. In about forty years, from the first generation of mobile systems, $1 \mathrm{G}$, to the current $5 \mathrm{G}$, we have witnessed an extraordinary evolution, or more appropriately a revolution, of the communication technologies that had a profound impact on different spheres of our life: social, cultural, economic and political. Just think how the advent of the smartphones made our daily social interactions much more virtual than real, or how from our pocket we can access to real-time information from all over the world which affects our cultural and political beliefs. For its effects on our daily lives, the Information and Communication revolution is perhaps as significant as the Industrial revolution and the Digital revolution.

The nature of the mobile communication has also changed over these years and, with that, also the needs of the mobile users. Initially, mobile systems were analog and been conceived to exclusively handle voice traffic. Nowadays, communications are digital and mainly involving data (e.g., video/audio streaming, social networking, web browsing, file sharing, etc.) rather than voice traffic. Indeed, mobile data surpassed voice on a global basis during December of 2009 [1]. Along with the increase of the data-processing capabilities and the storage capacity of the devices, and the advent of the mobile application ecosystem, the users are becoming increasingly demanding and data-hungry.

\section{The 5G Magic Triangle}

5G, also known as New Radio (NR) [2], has been conceived and designed to offer much higher data rates than its predecessors (in the order of $\mathrm{Gbps}^{1}, 1000 \times$ with

\footnotetext{
${ }^{1}$ Gigabit per second is a unit of data transfer rate equal to $10^{9}$ bits per second.
} 
respect to $4 \mathrm{G}[3]$ ), to cope with a seemingly ever increasing demand for data services. In fact, by the end of $2025,8.9$ billion of mobile subscriptions are forecast ( 2.8 billion are $5 \mathrm{G}$ ), out of which 88 percent will be for enhanced mobile broadband (eMBB) [4]. This is not the only use case $5 \mathrm{G}$ is targeting, but it is one out of three cornerstones of the magic triangle along with ultra-reliable low-latency communication (URLLC), and massive machine-type communication (mMTC) [5]. URLLC enables real-time applications characterized by time-critical communications, such as autonomous vehicles [6,7], remote medical operations [8], industrial automation control [9, $10]$, the "tactile" Internet $[11,12]$. mMTC enables connectivity between a vast number of miscellaneous devices and sensors [13,14]. It is just a $5 \mathrm{G}$ lingo for the well-known Internet of Things (IoT), namely a network of heterogeneous devices which are capable to exchange data without human intervention [15]. Supporting simultaneously these use cases, characterized by diverse quality-of-service (QoS) requirements, demands an efficient use (and re-use) of the network infrastructure and resources. Hence, $5 \mathrm{G}$ also focuses on how the actual physical network must be designed and sliced into multiple logical networks dedicated to different services (or users) based on their requirements. This is the principle of the so-called network slicing [16-18] which is attainable by using software-defined networking (SDN) and network function virtualization (NFV) [19].

\section{Achieving the eMBB Requirements: The "Big Three"}

The eMBB is unquestionably the use case with the largest market share, and what networking and telecommunication companies mainly looked at since day one of the $5 \mathrm{G}$ development journey. Increasing the data rate (a.k.a. throughput) delivered to the users is also the leitmotif of the studies in this thesis.

The area throughput is defined as the amount of information bits that can be successfully delivered to a specific area, per unit time. It is measured in $\mathrm{bit} / \mathrm{s} / \mathrm{km}^{2}$ and given by

$$
T_{\text {area }}=B[\mathrm{~Hz}] \cdot D\left[\text { cell } / \mathrm{km}^{2}\right] \cdot \mathrm{SE}[\mathrm{bit} / \mathrm{s} / \mathrm{Hz} / \text { cell }] \text {, }
$$

where $B$ is the bandwidth, $D$ is the cell density, namely the number of cells per $\mathrm{km}^{2}$, and SE is the spectral efficiency per cell, which is defined as the amount of information bits per second sent to a cell, over one $\mathrm{Hz}$ of bandwidth. Hence, an increase of the area throughput can be achieved by increasing these three factors. ${ }^{2}$

Using more bandwidth might appear the most straightforward solution to boost the throughput. However, the spectrum is a limited resource, globally shared

\footnotetext{
${ }^{2}$ Although related to each other, these three components can be considered independent as a first-order approximation [20].
} 
among different technologies and thereby quite costly. Little frequency resources are available below $6 \mathrm{GHz}$, where the most of the current wireless technologies operate, hence the interest in the millimeter wave (mmWave) bands, namely the range $30-300 \mathrm{GHz}$ where the wavelength is in the order of the millimeter. However, the electromagnetic signals are subject to many propagation issues over these frequencies, including large attenuation due to the distance (known as path loss) and low ability to penetrate objects (i.e., sensitiveness to blocking), which makes mmWave appealing only for short-range, line-of-sight (LoS) communications.

Increasing the cell density entails shrinking the cells by deploying more base stations (BSs) in the network. This approach has been aggressively exploited in the past decades [21,22]. Cellular networks are nowadays deployed in hierarchical tiers, where macro cells cover large areas (radius in the order of hundreds of meters), providing service to all the users therein and to the sub-tiers, namely small cells covering smaller area (with radius down to tens of meters). By densifying the network, any user is more likely to stand close to a BS and thereby to experience an excellent service. Small cells can be even designed to serve only one user which does not have to share any resource with other users. On the other hand, network densification entails high deployment and maintenance costs. Importantly, as we densify the network the inter-cell interference becomes the major bottleneck, which leads to a throughput degradation $[23,24]$.

The SE can be increased by using multiple transmitting/receiving antennas at both the BS and the user side. This is known as MIMO (multiple-input multipleoutput) technology [25-27]. Co-located and distributed MIMO systems will be discussed in detail in Chapter 2 and Chapter 3, respectively.

\section{MIMO Systems: If Less is Good, More is Better}

Having more transmitting/receiving antennas enables to increase the transmission/reception directivity. Let us focus on the transmission, but similar arguments apply to the reception. By appropriately time-synchronizing the single transmissions at each antenna, one can direct the total signal energy to any desired direction. By doing so, the signal components add up constructively over the air, generating a beam, and a receiver located in the intended direction observes a signal $M$-times stronger than the signal transmitted at each antenna. This technique is called beamforming [28] and $M$ represents the beamforming gain. The beamforming gain is equal to the number of the transmitting antennas, provided that they are half-wavelength apart. Hence, the more the antennas, the narrower the beam, the larger the beamforming gain and the SE. Importantly, in any other undesired direction we have destructive interference, thus receivers in these locations will experience little interference. 
This ability to focus a transmission on a single point in space enables to serve multiple users in the same time-frequency resources and separate them in the spatial domain. This is known as spatial multiplexing (or space division multiple access, SDMA) [29] and the resulting gain in SE is referred to as spatial multiplexing gain.

The use of multiple transmitting/receiving antennas does not only yield a SE increase. As a signal goes through different, possibly independent channels, it is subject to diverse alterations. This diversity boosts the quality of the signal at the receiver, and enhances the robustness and reliability of the link. This is known as spatial diversity gain [30].

Although various MIMO concepts have been around for decades, LTE (which stands for Long Term Evolution, the commercial name for $4 \mathrm{G}$ ) has been the very first mobile standard fully implementing multiuser MIMO technology, since 2010. LTE BSs can be equipped with up to 8 antenna ports covering 120 degree horizontal sector, for a total of 24 antenna ports. The aforementioned performance gains are noticeable in LTE, but come at the expense of complicated signal processing and the need of substantial channel estimation resources, which constitute, in fact, the inherent bottleneck of multiuser MIMO technology. Yet, massive gains are needed to fulfill the $5 \mathrm{G}$ eMBB requirements.

\section{Massive MIMO: The Ultimate MIMO Embodiment}

Massive MIMO [20,31-33], also known as large-scale antenna system, scales up multiuser MIMO [34]. This technology, whose concept has been introduced by the seminal paper [35], consists in equipping BSs with a number of antennas much larger than the number of active users per time-frequency resource. ${ }^{3}$ In this "massive MIMO regime" the BS provides extraordinary amounts of beamforming gain and spatial diversity.

Importantly, the excess of BS antennas compared to the users triggers a phenomenon called favorable propagation [37], namely users' channels become (approximately) mutually orthogonal as the number of BS antennas grows, which makes the inter-user interference vanish and facilitates an aggressive spatial multiplexing of the users. The favorable propagation also makes linear signal processing schemes, such as maximum ratio (MR) and zero forcing (ZF), effective and nearly optimal, hence considerably simplifying the circuitry complexity.

Interestingly, massive MIMO can offer, in most of the propagation environments, another benefit known as channel hardening [38,39]. The effective scalar

\footnotetext{
${ }^{3}$ The optimal ratio between the number of BS antennas and users depends on many factors, such as propagation environment and amount of available time-frequency resources. A common misconception is that at least an order of magnitude more antennas than users is needed [36].
} 
channel seen by any user is nearly deterministic as the effect of the small-scale fading is averaged out over the many channel observations, by virtue of the law of large numbers. The time-division duplex (TDD) operation is advocated in massive MIMO to significantly save radio resources, as the channel estimates in the uplink are also valid for the downlink. Channel reciprocity and channel hardening make the estimation of the instantaneous downlink channel superfluous, as the users can effectively rely on the channel statistics for data decoding [40]. As a consequence, the amount of resources dedicated to the channel estimation is independent of the number of antennas, but rather scales with the number of users. This makes massive MIMO a scalable technology.

Last but not least, as the law of large numbers comes to play, elegant closedform expressions for the achievable SE can be derived to predict the system performance $[20,33,41]$. This enables optimization of the spectral and energy efficiency as well as simplification of resource allocation tasks such as power control [20,33,42-49] and pilot assignment [48, 50-53].

Current massive MIMO implementations have corroborated the theoretical studies, and today Massive MIMO is a key enabler of $5 \mathrm{G} \mathrm{NR}$, as correctly predicted (see e.g., $[3,54]$ ). Massive MIMO BSs are being deployed all over the world, and telecommunication companies are competing to set new records on the achievable SE. The world's first commercial 5G NR radio for massive MIMO is the AIR 6468 by Ericsson, showcased in 2017, which uses 64 transmit and 64 receive antennas and operates in the sub- $6 \mathrm{GHz}$ bands [55]. Afterwards, other companies such as Huawei, Nokia, ZTE, and Facebook have demonstrated how to attain impressive SE by using massive MIMO BSs equipped with 128 antennas. This has been a severe blow for those skeptical arguing that the entire massive MIMO theory solely relies on asymptotic results (i.e., for infinite number of BS antennas).

\section{Cell-free massive MIMO: The Ultimate Network MIMO Embodiment}

Cell-free massive MIMO $[56,57]$ is the concept that potentially combines the "big three" $5 \mathrm{G}$ technologies described earlier (ultra-densification, mmWave and massive MIMO) and can theoretically provide unprecedented levels of throughput.

In cell-free massive MIMO, many antennas are geographically distributed rather than co-located in a BS. The antennas, hereafter referred to as access points (APs), operate jointly, synchronously and coherently to serve the users in the same timefrequency resources. The APs act as a single massive MIMO BS, coordinated by one or more central processing units (CPUs) through a fronthaul network. This signal co-processing at multiple distributed APs yields three main benefits in addition to the beamforming and spatial multiplexing gain:

- Macro-diversity gain. This gain is a direct consequence of the aggressive 
deployment densification. Macro-diversity occurs whenever the distance between the transmitters is much longer than the wavelength, and thereby geography comes into play by enriching the level of spatial diversity. Moving the APs to the proximity of the users leads to a substantial reduction of the path loss and the shadowing effects. The reliability of the link is also improved as each user is surrounded by serving APs and the probability that each user is obstructed from every direction is quite low. These features make cell-free massive MIMO suitable for mmWave communications. The macro-diversity gain manifests in larger channel gains.

- Inter-cell interference mitigation. This is a direct consequence of the signal co-processing, which enables to turn the inter-cell interference into useful signal. All the APs serve all the users in their surroundings, thereby erasing the cell boundaries when transmitting/receiving data, hence the term cellfree. Therefore, cell-free massive MIMO significantly outperforms small cell networks $[58,59]$, which are inter-cell interference limited and do not provide macro-diversity.

- More uniform quality of service among the users. This is a direct consequence of the two previous points. Essentially, in a cell-free system there are no cell-edge users but rather cell-center users experiencing roughly the same good channel conditions.

The enormous potential of cooperative multi-antenna transmission/reception techniques has been explored since the early 2000s [60], leading the way to a parallel line of research on distributed MIMO networks [61-63], also known as network MIMO $[64,65]$. There have been different flavors of network MIMO with different names: distributed antenna systems (DAS) [66], cooperative MIMO [67, 68], virtual MIMO [69] and coordinated multipoint (CoMP) [70-72]. They all shared the same basic concept of mitigating the inter-cell interference by grouping multiple cells in fixed cooperation clusters. However, this just shifts the interference management from the cell level to the cluster level, and the inter-cluster interference constitutes a fundamental limit of cooperation [73]. Indeed, CoMP-included in LTE-Advanced (4.5G)-has not met the initial expectations [74].

Conversely, there are at least five main reasons why cell-free massive MIMO, unlike CoMP, is deemed to be a beyond-5G key enabling technology [75-79]: (i) the massive MIMO baseline operation, (ii) the user-centric perspective set up by dynamic cooperation clustering, (iii) predictable performance which gives room to a simplified resource optimization, (iv) the potential marriage with mmWave, and $(v)$ the emerging cost-efficient solutions for the deployments. These aspects will be discussed in detail in Chapter 3 . 
On the other hand, in order to implement such a user-centric philosophy, cell-free massive MIMO requires a widespread and costly architecture, accurate synchronization and coordination among the APs, and simple but effective resource allocation schemes to minimize the accompanying signalling overhead. Importantly, in its canonical form, cell-free massive MIMO is not a scalable system, namely it is not able to handle a growing amount of users and APs in the system. Understanding which network tasks should be implemented either in a distributed manner at each APs or in centralized manner at the CPU is crucial in order to preserve the system scalability and make cell-free massive MIMO practical.

\subsection{Contributions of the Thesis}

This is a composite thesis consisting of an introductory part and a collection of papers. The introductory part is a journey through the basics of MIMO technology. It gives a description of co-located and distributed MIMO systems, from point-topoint MIMO to massive MIMO (Chapter 2) and from network MIMO to cell-free massive MIMO (Chapter 3), respectively. The second part consists of papers which mainly focus on three aspects of cell-free massive MIMO: scalability, signal processing and power control.

More specifically, Paper A is an overview article that points out strengths and weaknesses of cell-free massive MIMO with respect to existing cellular technologies and distributed MIMO systems. It also touches aspects concerning AP clock synchronization and proposes a practical cost-efficient implementation, referred to as Radio Stripes [80].

Paper B proposes a scalable cell-free massive MIMO framework [81], including data transmission and power control strategies. Unlike prior studies-which consider cell-free massive MIMO as an infinitely large single cell served by one CPU-here it is considered a realistic scenario where multiple CPUs serve disjoint subset of APs.

Paper C, Paper D and Paper E focus on signal processing and power control in the downlink, while always keeping an eye on scalability. More specifically, Paper $\mathrm{C}$ shows how the SE can be further improved by proposing fully distributed and scalable precoding schemes which provide additional interference cancelation gain [82]. Paper D emphasizes the benefits of using pilot-based downlink training to cope with the lower degree of channel hardening [83] in cell-free massive MIMO compared to co-located massive MIMO. While, Paper E proposes alternative conjugate beamforming (a.k.a. maximum ratio) schemes to boost the channel hardening and make the user data decoding, relying solely on statistical knowledge of the channels, more effective. A leitmotif connecting these works is the max-min 
fairness power control. As mentioned earlier, by nature, cell-free massive MIMO provides excellent uniform service to the users. Max-min fairness is therefore advocated as maximizing the minimum SE does not result in a significant penalty for the users with higher SE, unlike in co-located massive MIMO.

Finally, a common assumption in these papers is that the channel statistics (i.e., path loss and shadow fading) are known a priori at the APs, and thereby Bayesian channel estimation methods can be performed. In this regard, Paper F proposes an a-priori-assumption-free channel estimation method.

\section{Paper A: Ubiquitous cell-free massive MIMO communications}

Authored by: Giovanni Interdonato, Emil Björnson, Hien Quoc Ngo, Pål Frenger and Erik G. Larsson.

Published in EURASIP Journal on Wireless Communications and Networking, vol. 2019, no. 1, p. 197, August 2019.

Abstract: Since the first cellular networks were trialled in the 1970s, we have witnessed an incredible wireless revolution. From $1 \mathrm{G}$ to $4 \mathrm{G}$, the massive traffic growth has been managed by a combination of wider bandwidths, refined radio interfaces, and network densification, namely increasing the number of antennas per site. Due its cost-efficiency, the latter has contributed the most. Massive MIMO (multiple-input multiple-output) is a key $5 \mathrm{G}$ technology that uses massive antenna arrays to provide a very high beamforming gain and spatially multiplexing of users, and hence, increases the spectral and energy efficiency. It constitutes a centralized solution to densify a network, and its performance is limited by the inter-cell interference inherent in its cell-centric design. Conversely, ubiquitous cell-free Massive MIMO refers to a distributed Massive MIMO system implementing coherent user-centric transmission to overcome the inter-cell interference limitation in cellular networks and provide additional macro-diversity. These features, combined with the system scalability inherent in the Massive MIMO design, distinguishes ubiquitous cell-free Massive MIMO from prior coordinated distributed wireless systems. In this article, we investigate the enormous potential of this promising technology while addressing practical deployment issues to deal with the increased back/front-hauling overhead deriving from the signal co-processing. 


\section{Paper B: Scalability Aspects of Cell-Free Massive MIMO}

Authored by: Giovanni Interdonato, Pål Frenger and Erik G. Larsson.

Published in the proceedings of 2019 IEEE International Conference on Communications (ICC), pp. 1-6, May 2019.

Abstract: Ubiquitous cell-free massive MIMO (multiple-input multiple-output) combines massive MIMO technology and user-centric transmission in a distributed architecture. All the access points (APs) in the network cooperate to jointly and coherently serve a smaller number of users in the same time-frequency resource. However, this coordination needs significant amounts of control signalling which introduces additional overhead, while data co-processing increases the back/fronthaul requirements. Hence, the notion that the "whole world" could constitute one network, and that all APs would act as a single base station, is not scalable. In this study, we address some system scalability aspects of cell-free massive MIMO that have been neglected in literature until now. In particular, we propose and evaluate a solution related to data processing, network topology and power control. Results indicate that our proposed framework achieves full scalability at the cost of a modest performance loss compared to the canonical form of cell-free massive MIMO.

\section{Paper C: Local Partial Zero-Forcing Precoding for Cell-Free Massive MIMO}

Authored by: Giovanni Interdonato, Marcus Karlsson, Emil Björnson and Erik G. Larsson.

Published in IEEE Transactions on Wireless Communications, vol. 19, no. 7, pp. 4758-4774, July 2020.

Abstract: Cell-free Massive MIMO (multiple-input multiple-output) is a promising distributed network architecture for 5G-and-beyond systems. It guarantees ubiquitous coverage at high spectral efficiency (SE) by leveraging signal co-processing at multiple access points (APs), aggressive spatial user multiplexing and extraordinary macro-diversity gain.

In this study, we propose two distributed precoding schemes, referred to as local partial zero-forcing (PZF) and local protective partial zero-forcing (PPZF), that further improve the spectral efficiency by providing an adaptable trade-off between interference cancelation and boosting of the desired signal, with no additional front-hauling overhead, and implementable by APs with very few antennas.

We derive closed-form expressions for the achievable SE under the assumption of independent Rayleigh fading channel, channel estimation error and pilot contamination. PZF and PPZF can substantially outperform maximum ratio transmission 
and zero-forcing, and their performance is comparable to that achieved by regularized zero-forcing (RZF), which is a benchmark in the downlink. Importantly, these closed-form expressions can be employed to devise optimal (long-term) power control strategies that are also suitable for RZF, whose closed-form expression for the SE is not available.

\section{Paper D: Downlink Training in Cell-Free Massive MIMO: A Blessing in Disguise}

Authored by: Giovanni Interdonato, Hien Quoc Ngo, Pål Frenger and Erik G. Larsson.

Published in IEEE Transactions on Wireless Communications, vol. 18, no. 11, pp. 5153-5169, November 2019.

Abstract: Cell-free Massive MIMO (multiple-input multiple-output) refers to a distributed Massive MIMO system where all the access points (APs) cooperate to coherently serve all the user equipments (UEs), suppress inter-cell interference and mitigate the multiuser interference. Recent works demonstrated that, unlike co-located Massive MIMO, the channel hardening is, in general, less pronounced in cell-free Massive MIMO, thus there is much to benefit from estimating the downlink channel.

In this study, we investigate the gain introduced by the downlink beamforming training, extending the previously proposed analysis to non-orthogonal uplink and downlink pilots. Assuming single-antenna APs, conjugate beamforming and independent Rayleigh fading channel, we derive a closed-form expression for the per-user achievable downlink rate that addresses channel estimation errors and pilot contamination both at the AP and UE side. The performance evaluation includes max-min fairness power control, greedy pilot assignment methods, and a comparison between achievable rates obtained from different capacity-bounding techniques. Numerical results show that downlink beamforming training, although increases pilot overhead and introduces additional pilot contamination, improves significantly the achievable downlink rate. Even for large number of APs, it is not fully efficient for the UE relying on the statistical channel state information for data decoding. 


\section{Paper E: Enhanced Normalized Conjugate Beamforming for Cell-Free Massive MIMO}

Authored by: Giovanni Interdonato, Hien Quoc Ngo and Erik G. Larsson

Submitted to IEEE Transactions on Communications.

Abstract: In cell-free massive multiple-input multiple-output (MIMO) the fluctuations of the channel gain from the access points to a user are large due to the distributed topology of the system. Because of these fluctuations, data decoding schemes that treat the channel as deterministic perform inefficiently.

A way to reduce the channel fluctuations is to design a precoding scheme that equalizes the effective channel gain seen by the users. Conjugate beamforming (CB) poorly contributes to harden the effective channel at the users. In this work, we propose a variant of $\mathrm{CB}$ dubbed enhanced normalized $\mathrm{CB}(\mathrm{ECB})$, in that the precoding vector consists of the conjugate of the channel estimate normalized by its squared norm. We derive an exact closed-form expression for an achievable downlink spectral efficiency (SE), accounting for channel estimation errors, pilot reuse and user's lack of channel state information (CSI), under the assumption of independent Rayleigh fading channels. We also devise an optimal max-min fairness power allocation based only on large-scale fading quantities. ECB greatly boosts the channel hardening enabling the users to reliably decode data relying only on statistical CSI. As the provided effective channel is nearly deterministic, acquiring CSI at the users does not yield a significant gain.

\section{Paper F: Self-Learning Detector for the Cell-Free Massive MIMO Uplink: The Line-of-Sight Case}

Authored by: Giovanni Interdonato, Pål Frenger and Erik G. Larsson

Published in the proceedings of 2020 IEEE 21st International Workshop on Signal Processing Advances in Wireless Communications (SPAWC), pp. 1-5, May 2020.

Abstract: The precoding in cell-free massive multiple-input multiple-output (MIMO) technology relies on accurate knowledge of channel responses between users (UEs) and access points (APs). Obtaining high-quality channel estimates in turn requires the path losses between pairs of UEs and APs to be known. These path losses may change rapidly especially in line-of-sight environments with moving blocking objects. A difficulty in the estimation of path losses is pilot contamination, that is, simultaneously transmitted pilots from different UEs that may add up destructively or constructively by chance, seriously affecting the estimation quality (and hence the eventual performance). A method for estimation of path losses, along with an accompanying pilot transmission scheme, is proposed that works for both Rayleigh fading and line-of-sight channels and that significantly 
G. Interdonato, H. Q. Ngo, E. G. Larsson and P. Frenger, "On the performance of cell-free massive MIMO with short-term power constraints," in 2016 IEEE 21st International Workshop on Computer Aided Modelling and Design of Communication Links and Networks (CAMAD), Toronto, ON, 2016, pp. 225-230.

G. Interdonato, H. Q. Ngo, E. G. Larsson and P. Frenger, "How Much Do Downlink Pilots Improve Cell-Free Massive MIMO?," in 2016 IEEE Global Communications Conference (GLOBECOM), Washington, DC, 2016, pp. 1-7.

G. Interdonato, P. Frenger and E. G. Larsson, "Utility-based Downlink Pilot Assignment in Cell-Free Massive MIMO," in 2018 22nd International ITG Workshop on Smart Antennas (WSA), Bochum, Germany, 2018, pp. 1-8.

G. Interdonato, M. Karlsson, E. Björnson and E. G. Larsson, "Downlink Spectral Efficiency of Cell-Free Massive MIMO with Full-Pilot Zero-Forcing," in 2018 IEEE Global Conference on Signal and Information Processing (GlobalSIP), Anaheim, CA, USA, 2018, pp. 1003-1007.

improves performance over baseline state-of-the-art. The salient feature of the pilot transmission scheme is that pilots are structurally phase-rotated over different coherence blocks (according to a pre-determined function known to all parties), in order to create an effective statistical distribution of the received pilot signal that can be efficiently exploited by the proposed estimation algorithm.

\subsection{Excluded Papers}

The papers in Table 1 are not included in this thesis as they are preliminary versions of the included papers, and thereby redundant. 


\section{Chapter 2}

\section{Co-located MIMO Systems}

In this chapter, we go through the evolution of the co-located MIMO systems, exploring the basics of point-to-point MIMO, multiuser MIMO and massive MIMO technology. The focus of this chapter is to highlight the three main benefits of MIMO: beamforming gain, spatial diversity and spatial multiplexing, but above all to discuss why massive MIMO constitutes a breakthrough technology for providing high spectral efficiency.

\subsection{Preliminaries}

To describe the benefits of having multiple antennas at the transmitter and receiver, we need to recall the analysis of the SISO (single-input single-output) system, which will serve as reference, and choose a performance metric of interest.

\subsubsection{Channel Capacity and Outage Probability}

Two fundamental performance metrics for communication systems are the channel capacity and the outage probability. The former gives a measure of the maximum amount of information which can be reliably conveyed over a channel, while the latter gives a measure of the reliability of the communication link.

More rigorously, the channel capacity, $C$, is the maximum number of bits per symbol which can be transmitted over a channel with arbitrarily low error probability, as the number of transmitted symbols tends to infinity [84, 85]. Hence, the capacity constitutes an upper bound on the rate, and any number of bits per symbol below the capacity is referred to as an achievable rate (or SE).

In general, obtaining closed-form expressions for the exact channel capacity is not an easy task. In this thesis, we use different bounding techniques (along 
with reasonable approximations) to derive insightful achievable rate expressions in closed form.

The outage probability is a meaningful performance metric when the transmitter cannot compute the capacity and, as a consequence, does not know how to encode the data to achieve it. It is the probability that an unsuccessful communication occurs because of high error probability. This happens when the transmitter selects a transmission rate that is larger than the actual channel capacity. Hence, for a given rate $R$, the outage probability is given by $P_{\text {out }}(R)=\operatorname{Pr}\{R>C\}$.

\subsubsection{SISO Channel}

Let us consider a SISO channel. The signal at the receiver is given by ${ }^{1}$

$$
y=g x+n,
$$

where $g$ is the channel response, $x$ is the transmitted data symbol and $n$ is additive Gaussian noise with variance $\sigma^{2}$. We assume that $g$ is known at the receiver but not at the transmitter, $x$ is independent of the noise, and the maximum transmit power is $\rho$.

Depending on how quickly $g$ varies over time, we have: (i) no fading, if $g$ is a deterministic constant; (ii) slow fading, if the channel variations are much slower than the symbol rate and thereby $g$ takes only one random realization throughout the transmission; (iii) fast fading, if the channel varies at the symbol rate and thereby $g$ takes many random realizations throughout the transmission.

For a SISO channel with slow fading, Shannon theory yields the following capacity expression, conditioned on a given realization of $g$,

$$
C=\log _{2}\left(1+|g|^{2} \mathrm{SNR}\right) \quad \mathrm{bit} / \mathrm{symbol},
$$

which is achieved when $x$ is circularly symmetric Gaussian random variable with variance $\rho, x \sim C \mathcal{N}(0, \rho)$. The signal-to-noise ratio (SNR) equals $\rho / \sigma^{2}$.

Since the transmitter does not know the channel, an outage might occur with some probability. In this case, the outage probability is

$$
P_{\text {out }}(R)=\operatorname{Pr}\left\{R>\log _{2}\left(1+|g|^{2} \mathrm{SNR}\right)\right\}=\operatorname{Pr}\left\{|g|^{2}<\frac{2^{R}-1}{\mathrm{SNR}}\right\} .
$$

For instance, if $g \sim \mathcal{C N}(0,1)$, then $|g|^{2}$ is exponential distributed and

$$
P_{\text {out }}(R)=1-\exp \left(-\frac{2^{R}-1}{\mathrm{SNR}}\right) \approx \frac{2^{R}-1}{\mathrm{SNR}},
$$

\footnotetext{
${ }^{1}$ Throughout the thesis, the complex baseband representation of the signal is used.
} 
where the approximation is only valid at high-SNR regime, by using the fact that $e^{-x} \approx 1-x$, for $x$ small.

In a fast fading scenario, the capacity is more properly referred to as the ergodic capacity to emphasize that the channel response originates from an ergodic random process. The ergodic capacity is obtained by taking the expected value of the (conditional) capacities with respect to the channel response. For the SISO fading channel, the ergodic capacity is

$$
C=\mathbb{E}\left\{\log _{2}\left(1+|g|^{2} \mathrm{SNR}\right)\right\} \quad \text { bit/symbol. }
$$

Importantly, the ergodic capacity is a deterministic quantity which can be computed by the transmitter without knowing the channel realizations but simply exploiting the knowledge of the channel statistics. Therefore, the transmitter can set up the transmission rate accordingly and enable outage-free communication.

The outage probability is a meaningful measure only when the transmitter does not know the channel in a slow fading scenario. These are the assumptions that we consider when analyzing the outage probability in Section 2.2 and Section 2.3. While, for the sake of simplicity, deterministic channels are considered when analyzing the capacity in Section 2.2 and Section 2.3, although similar conclusions also hold for slow fading and, provided that $M$ is large, for fast fading.

\subsection{Benefits of Using Multiple Antennas}

By adding more antennas at one end of the communication link, channel capacity and outage probability can be improved as a result of the beamforming gain and diversity gain, respectively. These performance gains are presented analytically next by considering simple examples.

\subsubsection{Beamforming Gain}

Consider a single-input multiple-output (SIMO) system where the receiver is equipped with $M$ antennas. The received signal is given by

$$
\boldsymbol{y}=\boldsymbol{g} x+\boldsymbol{n},
$$

where $\boldsymbol{y}=\left[y_{1} \cdots y_{M}\right]^{\top}, \boldsymbol{g}=\left[g_{1} \cdots g_{M}\right]^{\top}$ and $\boldsymbol{n}=\left[n_{1} \cdots n_{M}\right]^{\top}$. The channel vector $\boldsymbol{g}$ is known at the receiver but not at the transmitter, $\boldsymbol{n} \sim \mathcal{C N}\left(\mathbf{0}, \sigma^{2} \boldsymbol{I}_{M}\right)$ is independent of $x$. The receiver can exploit the knowledge of the channel by properly combining the $M$ signal observations. The channel capacity is achieved for $x \sim C \mathcal{N}(0, \rho)$ by post-processing the signal as follows $[25,26]$

$$
\frac{\boldsymbol{g}^{\mathrm{H}}}{\|\boldsymbol{g}\|} \boldsymbol{y}=\|\boldsymbol{g}\| x+\frac{\boldsymbol{g}^{\mathrm{H}} \boldsymbol{n}}{\|\boldsymbol{g}\|}
$$


which is known as maximum-ratio combining (MRC) and gives

$$
C=\log _{2}\left(1+\|\boldsymbol{g}\|^{2} \mathrm{SNR}\right) \quad \text { bit/symbol. }
$$

The term $\|\boldsymbol{g}\|^{2}$ in (1) represents the beamforming gain, that is an increase of the effective SNR at the receiver compared to a SISO system. Since $\|\boldsymbol{g}\|^{2}$ is often proportional to $M$, the capacity scales logarithmically with $M$ at high SNR and linearly with $M$ at low SNR. ${ }^{2}$ Hence, the more antennas are used, the better the receiver can capture the power of the transmitted signal.

This exact capacity is also achievable by a multiple-input single-output (MISO) system with $M$ antennas at the transmitter, provided that it knows $\boldsymbol{g}$ and performs a pre-processing of the transmitted symbol (also known as precoding), dual to MRC, called maximum-ratio transmission (MRT). More specifically, MRT consists in precoding the data symbol by the conjugate of the channel vector (hence the alternative name conjugate beamforming). By doing so, a constructive interference of the $M$ signals at the receiver location is ensured, and the more antennas are used at the transmitter, the more power can be spatially focused to the receiver.

\subsubsection{Spatial Diversity Gain}

Consider the SIMO fading channel $\boldsymbol{g} \sim \mathcal{C N}\left(\mathbf{0}, \boldsymbol{I}_{M}\right)$ and MRC at the receiver. Under this assumption, $\|\boldsymbol{g}\|^{2}$ has a scaled $\chi^{2}$-distribution, and at high SNR, the outage probability is [26]

$$
P_{\text {out }}(R)=\operatorname{Pr}\left\{\|\boldsymbol{g}\|^{2}<\frac{2^{R}-1}{\mathrm{SNR}}\right\} \approx\left(\frac{2^{R}-1}{\mathrm{SNR}}\right)^{M} \frac{1}{M !} .
$$

Hence, $P_{\text {out }}$ is proportional to $\mathrm{SNR}^{-M}$, meaning that for every $10 \mathrm{~dB}$ that the SNR increases, $P_{\text {out }}$ diminishes by a factor $1 / 10^{M}$. Plotting the outage probability in logarithmic scale against the SNR in $\mathrm{dB}$, as shown in Section 2.3, results in curves with slope $-M$. This is known as spatial (receive) diversity gain and $M$ is the diversity order. Hence, the more antennas are used, the more reliable is the communication.

In general, the spatial diversity gain can also be seen directly from the capacity expression. If the SNR is proportional to $\left|g_{1}\right|^{2}+\left|g_{2}\right|^{2}+\cdots+\left|g_{N}\right|^{2}$, with $N \leq M$, then a spatial diversity gain of order $N$ is achieved. The probability that all the independent random variables $\left|g_{n}\right|^{2}, n=1, \ldots, N$, are small (i.e., the probability

\footnotetext{
${ }^{2}$ For instance, $\|\boldsymbol{g}\|^{2}=M \beta$ if either the receiver or the transmitter is equipped with a uniform linear array (ULA) with $M$ antennas, and $\beta$ is the channel gain which depends on the distance between the transmitter and the receiver.
} 
for the receiver to be in deep fade) decreases by increasing the number of receive antennas. If $N=M$, full receive diversity gain is achieved.

Without going into details, a MISO system can achieve transmit diversity gain even without knowing the channel by employing a fixed precoding technique spanning multiple channel uses. This is known as space-time block coding (STBC) [86,87], and the Alamouti code [88] is the most popular STBC enabling full transmit diversity with $M=2$ transmit antennas.

\subsection{Point-to-Point MIMO}

Point-to-point MIMO dates back to the late 1990s [25, 89, 90], and simply consists in a communication between a transmitter and a receiver both equipped with multiple antennas. With respect to SIMO/MISO, having multiple antennas at both the ends of the link can, under certain circumstances, significantly improve the channel capacity and the outage probability.

\subsubsection{Multiplexing Gain}

Let us consider a point-to-point MIMO system with $M$ antennas at the receiver and $K$ antennas at the transmitter. The MIMO channel is

$$
\boldsymbol{y}=G x+\boldsymbol{n},
$$

where $G$ is the $M \times K$ channel matrix with elements $\left\{g_{m k}\right\}, \boldsymbol{y}=\left[y_{1} \cdots y_{M}\right]^{\top}$, $\boldsymbol{x}=\left[x_{1} \cdots x_{K}\right]^{\top}$ and $\mathbb{E}\left\{\|x\|^{2}\right\}=\rho, \boldsymbol{n}=\left[n_{1} \cdots n_{M}\right]^{\top}$ and $\boldsymbol{n} \sim \mathcal{C N}\left(\mathbf{0}, \sigma^{2} \boldsymbol{I}_{M}\right)$.

Under the assumption of deterministic channel and channel state information (CSI) knowledge at the receiver but not at the transmitter, the capacity of point-topoint MIMO is given by $[25,26,33]$

$$
C=\log _{2}\left|I_{M}+\frac{\mathrm{SNR}}{K} G G^{\mathrm{H}}\right| \quad \text { bit/symbol. }
$$

The matrix $G G^{\mathrm{H}}$ can be expanded in term of its eigenvalue decomposition: $G G^{\mathrm{H}}=$ $\boldsymbol{U} \Lambda U^{\mathrm{H}}$, where $\boldsymbol{U}$ is $M \times M$ unitary matrix, and $\Lambda$ is a $M \times M$ positive diagonal matrix whose diagonal elements are the eigenvalues of $G G^{\mathrm{H}},[\Lambda]_{i i}=\lambda_{i}, i=1, \ldots, M$. Hence, the capacity can be rewritten as

$$
\begin{gathered}
C=\log _{2}\left|\boldsymbol{I}_{M}+\frac{\mathrm{SNR}}{K} \boldsymbol{U} \boldsymbol{\Lambda} \boldsymbol{U}^{\mathrm{H}}\right| \stackrel{(a)}{=} \log _{2}\left|\boldsymbol{U}\left(\boldsymbol{I}_{M}+\frac{\mathrm{SNR}}{K} \boldsymbol{\Lambda}\right) \boldsymbol{U}^{\mathrm{H}}\right| \\
\stackrel{(b)}{=} \log _{2}\left|\boldsymbol{I}_{M}+\frac{\mathrm{SNR}}{K} \boldsymbol{\Lambda}\right|=\sum_{l=1}^{L} \log _{2}\left(1+\frac{\mathrm{SNR}}{K} \lambda_{l}\right),
\end{gathered}
$$


where $L=\operatorname{rank}\left(G G^{\mathrm{H}}\right) \leq \min (M, K)$ (i.e., the number of non-zero eigenvalues). In $(a)$ we utilize $U U^{\mathrm{H}}=I$, and in $(b)$ the fact that $\operatorname{det}(A B C)=\operatorname{det}(A) \cdot \operatorname{det}(B)$. $\operatorname{det}(\boldsymbol{C})=\operatorname{det}(\boldsymbol{B})$, as $\boldsymbol{A}, \boldsymbol{B}$, and $\boldsymbol{C}$ have equal size and the determinant of a unitary matrix is equal to 1 .

Equation (4) tells us that the point-to-point MIMO channel can be decomposed into $L$ parallel SISO channels, and its capacity is the summation of the single SISO capacities. $L$ is called multiplexing gain (or spatial degrees of freedom) as it is the maximum number of parallel data streams we can convey over the MIMO channel. $L$ depends on the propagation environment, and it is upper bounded by the minimum between the number of antennas at the transmitter and the receiver.

The capacity in (4) can be maximized if the transmitter knows $G$ by performing eigen-beamforming and waterfilling power control [25,26]. Eigen-beamforming consists in precoding the data symbols with the right eigenvectors of $G^{\mathrm{H}} G$. By doing so, $L$ parallel data streams are transmitted simultaneously but with specific spatial directivity: this is known as spatial multiplexing. Waterfilling consists in allocating the transmit power proportionally to the channel conditions: the stronger the channel gain is, the more power is allocated.

To quantify the gain in capacity of point-to-point MIMO over SIMO/MISO, let us consider a small example. Consider a point-to-point MIMO system where a transmitter and a receiver have the same number of antennas, $M=K$. Let the channel matrix $G$ be full rank and have identical singular values. ${ }^{3}$ Finally, for the sake of simplicity, let $\left|g_{m k}\right|^{2}=1, m=1, \ldots, M, k=1, \ldots, K$. Under these assumptions, we have $L=M, \lambda_{l}=\lambda=M, \forall l$. Hence, the capacity in (4) becomes

$$
C=M \log _{2}(1+\mathrm{SNR}) \quad \text { bit/symbol. }
$$

The pre-log factor in (5) represents the multiplexing gain and ensures a significant capacity increase. Indeed, we observe that the capacity of the point-to-point MIMO is $M$ times larger than the corresponding SISO capacity, which is $C=\log _{2}(1+\mathrm{SNR})$. With respect the SIMO/MISO capacity, the capacity gap increases with the SNR, as shown in Figure 1. In fact, at high SNR the capacity of point-to-point MIMO scales linearly with $M$, while the capacity of SIMO/MISO, $C=\log _{2}(1+M \cdot \mathrm{SNR})$, scales only logarithmically with $M$. Hence, the multiplexing gain has more impact on the capacity than the beamforming gain.

\subsubsection{Extra Spatial Diversity}

Point-to-point MIMO can also increase the order of spatial diversity. Consider i.i.d. Rayleigh (slow) fading channel, $g_{m k} \sim C \mathcal{N}(0,1)$. The outage probability, assuming

\footnotetext{
${ }^{3}$ This represents the best possible propagation environment. In this case, the capacities with and without CSI at the transmitter coincide.
} 


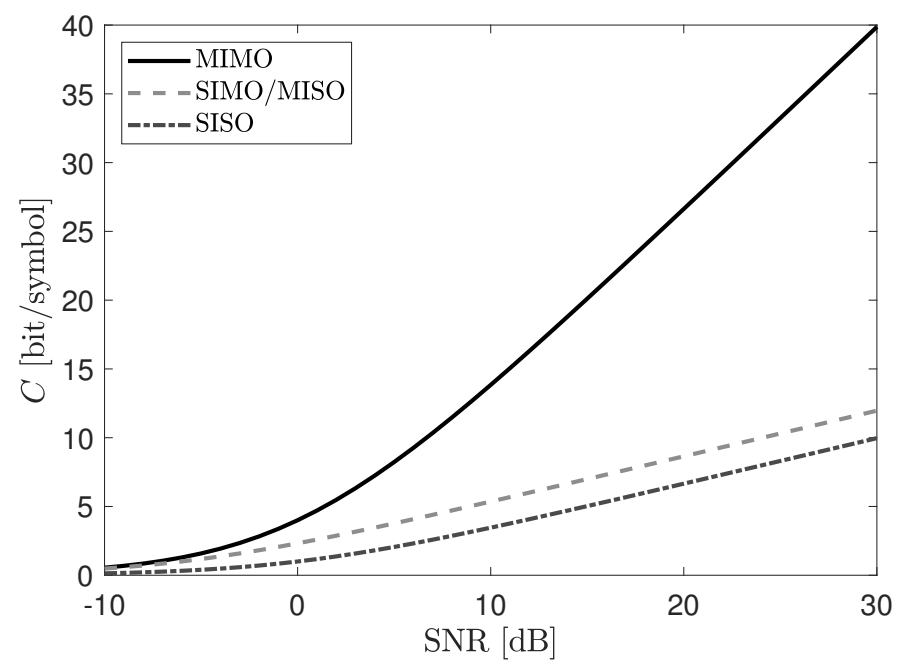

Figure 1: Capacity of MIMO, SIMO/MISO and SISO channel, assuming $M=K=4$, channel matrix $G$ with full rank and identical singular values, and $\left|g_{m k}\right|^{2}=1$.

no CSI at the transmitter, can be upper bounded as

$$
\begin{aligned}
P_{\text {out }}(R) & =\operatorname{Pr}\left\{R>\log _{2}\left|I_{M}+\frac{\mathrm{SNR}}{K} G G^{\mathrm{H}}\right|\right\} \\
& \stackrel{(a)}{\leq} \operatorname{Pr}\left\{R>\frac{\mathrm{SNR}}{K} \cdot \operatorname{Tr}\left(G G^{\mathrm{H}}\right) \log _{2}(e)\right\} \\
& \stackrel{(b)}{\leq} \operatorname{Pr}\left\{R>\mathrm{SNR} \cdot M \log _{2}(e) \cdot \max _{m, k}\left|g_{m k}\right|^{2}\right\} \\
& =\prod_{m=1}^{M} \prod_{k=1}^{K} \operatorname{Pr}\left\{\left|g_{m k}\right|^{2}<\frac{R}{\mathrm{SNR} \cdot M \log _{2}(e)}\right\} \\
& =\left[1-\exp \left(-\frac{R}{\mathrm{SNR} \cdot M \log _{2}(e)}\right)\right]^{M K} \\
& =\left(\frac{R}{M \log _{2}(e)}\right)^{M K} \cdot \mathrm{SNR}^{-M K}+\text { higher order terms. }
\end{aligned}
$$

The upper bounds in $(a)$ and $(b)$ are obtained by using the following result [33]

$$
\frac{C}{\log _{2}(e)} \leq \frac{\mathrm{SNR}}{K} \cdot \operatorname{Tr}\left(G G^{\mathrm{H}}\right) \leq \mathrm{SNR} \cdot M \cdot \max _{m, k}\left|g_{m k}\right|^{2},
$$

where $C$ is the capacity in (4). 


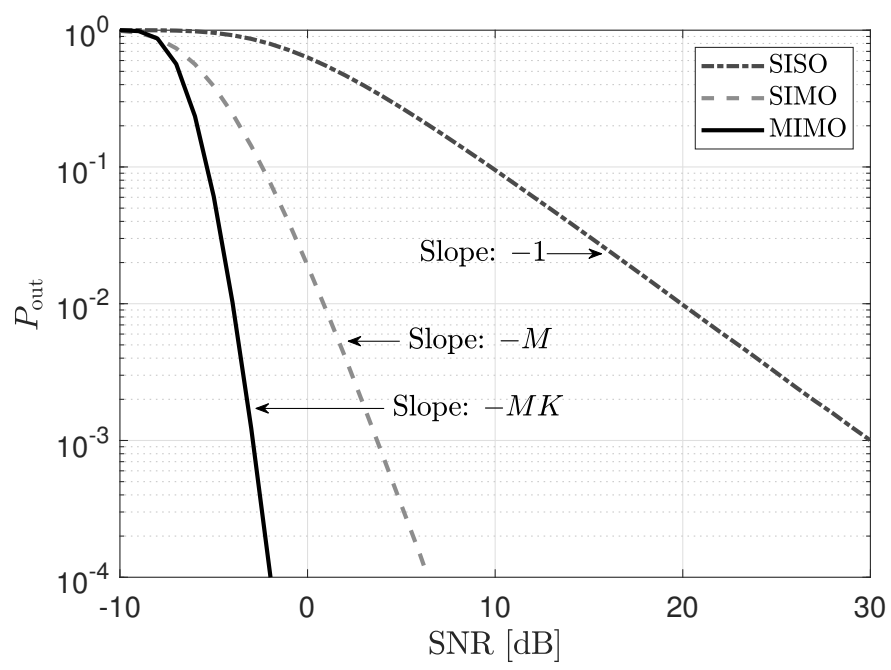

Figure 2: The exact outage probability of MIMO, SIMO and SISO channel, assuming $M=K=4$, i.i.d. Rayleigh fading, $g_{m k} \sim C \mathcal{N}(0,1)$, and $R=1 \mathrm{bit} / \mathrm{symbol}$.

Hence, point-to-point MIMO can theoretically increase $K$ times the spatial diversity order compared to a SIMO system. This increase of spatial diversity gain is clearly shown in Figure 2, where, at high SNR, the slope of the MIMO curve is $-M K$ while that of the SISO/SIMO curve equals $-M$.

\subsubsection{Practical Issues}

Point-to-point MIMO fulfills its potential under two conflicting conditions: (i) High SNR, which is the regime wherein the multiplexing gain manifests. In low-SNR regime point-to-point MIMO only provides beamforming gain [26], as shown in Figure 1; (ii) The propagation environment must support $\min (M, K)$ spatial degrees of freedom, that is $G$ must be full rank such that the multiplexing gain equals $\min (M, K)$.

These two conditions are unlikely to happen simultaneously. High SNR is likely to be achieved in propagation environments characterized by few dominant paths in LoS. The corresponding channel matrix is thereby rank deficient. The multiplexing gain even vanishes in practical scenarios where transmitter and receiver, both equipped with a ULA, are in LoS. Conversely, $G$ is full rank if the propagation environments is characterized by rich scattering. In such a NLoS scenario, the receiver is likely to experience low SNR and, as a consequence, solely beamforming gain.

Another limit that makes point-to-point MIMO unpractical and unsuitable 
for mobile wireless systems is the difficulty of making both $M$ and $K$ large to scale the performance accordingly. Battery-limited user terminals can afford only few antennas in order to keep both the power consumption and the hardware complexity low.

\subsection{Multiuser MIMO}

Multiuser MIMO overcomes the practical limitations of point-to-point MIMO described in Section 2.3.3.

Unlike point-to-point MIMO where the users are typically orthogonally multiplexed (e.g., in the time and frequency domains), in multiuser MIMO the users are served simultaneously in the same time-frequency resources but separated in the spatial domain (i.e., SDMA). This entails that, in the downlink, the BS must estimate the channels in order to perform precoding and enable the spatial multiplexing.

Multiuser MIMO is less sensitive to the propagation environment than pointto-point MIMO, and does require neither multi-antenna user terminals nor cooperation between the users. For its practicality and efficiency, multiuser MIMO has been a key enabling technology for LTE [91].

\subsubsection{Sum Capacity}

Serving the users over non-orthogonal resources causes inter-user interference, which degrades the performance. In this regard, interference cancellation techniques such as successive interference cancellation (SIC) [92] for the uplink, and dirty paper coding (DPC) [93] for the downlink, are necessary to achieve good spectral efficiency.

The uplink channel of multiuser MIMO, often called the multiple-access channel, can be modeled as the MIMO channel in (2). We consider $K$ single-antenna users and $M$ BS antennas. The coefficient $g_{m k}$ indicates the channel response from user $k$ to antenna $m$. Clearly, $G$ has now a different structure since each of its columns concerns a specific user. ${ }^{4}$ Another important difference between point-topoint MIMO and multiuser MIMO is that the users do not cooperate. Hence, the transmitted signals $x_{1}, \ldots, x_{K}$ are independent and possibly be subject to different power constraints. Therefore, when evaluating the channel capacity, it is reasonable to look at the sum capacity, namely the sum of the capacities achieved by the corresponding $K$ SIMO channels.

Under the assumption that the BS knows exactly $G$ (deterministic), and all the users transmit with the same power at a proper rate (to ensure no outage), the

\footnotetext{
${ }^{4}$ Each column of $G$ can be modeled as a SIMO channel.
} 
capacity of the multiple-access channel is given by $[26,94]$

$$
C_{\mathrm{ul}}^{\mathrm{sum}}=\log _{2}\left|I_{M}+\mathrm{SNR} \cdot G G^{\mathrm{H}}\right| \quad \text { bit/symbol, }
$$

which is essentially identical to the capacity of point-to-point MIMO in (3) (the total uplink transmit power in multiuser MIMO is $K$ times larger than that of point-to-point MIMO described in Section 2.3). The uplink sum capacity in (7) is achieved by using minimum mean-square error (MMSE) decoding with SICsee e.g., [33, Section C.4.1] for a simple proof with $K=2$ users. Importantly, (3) and (7) tell us that the lack of cooperation among the users does not cause any penalty in the performance. Moreover, since the users are independent and far apart, $G G^{\mathrm{H}}$ is likely to be full rank. Hence, a (spatial) multiplexing gain equal to $\min (M, K)$ is much easier to achieve compared to point-to-point MIMO. In general, the propagation environment is less stressful for multiuser MIMO, and the spatial degrees of freedom are higher as $K$ is not the number of antennas at the user terminal but the number of users.

Similar conclusions can be also drawn for the downlink (broadcast channel), which is modeled as

$$
\boldsymbol{y}=G^{\mathrm{H}} \boldsymbol{x}+\boldsymbol{n},
$$

where $G$ is defined as in (2), $\boldsymbol{y}=\left[y_{1} \cdots y_{K}\right]^{\top}, \boldsymbol{x}=\left[x_{1} \cdots x_{M}\right]^{\top}$ and $\mathbb{E}\left\{\|x\|^{2}\right\}=\rho$, $\boldsymbol{n}=\left[n_{1} \cdots n_{K}\right]^{\top}$ and $\boldsymbol{n} \sim \mathcal{C N}\left(\mathbf{0}, \sigma^{2} \boldsymbol{I}_{K}\right)$. The sum capacity, achievable by using DPC and MMSE precoding, is given by $[26,95,96]$

$$
C_{\mathrm{dl}}^{\text {sum }}=\max _{\substack{\left\{v_{k}\right\}, v_{k} \geq 0 \\ v_{1}+\cdots+v_{K} \leq 1}} \log _{2}\left|I_{M}+\mathrm{SNR} \cdot G D_{v} G^{\mathrm{H}}\right| \quad \text { bit/symbol, }
$$

where $D_{v}$ is a diagonal matrix with the power control coefficients $v_{1}, \ldots, v_{K}$ as diagonal entries-see e.g., [33, Section C.4.2] for a simple proof with $K=2$ users. This capacity is achieved under the assumption that $G$ is known at the BS, and thereby it may exceed the capacity of the corresponding point-to-point MIMO without CSI at the transmitter-which is given by (3) but replacing SNR/K with $\mathrm{SNR} / M$. Moreover, the capacity in (9) requires a convex optimization problem to be solved. In general, optimal precoding selection and power allocation in multiuser MIMO is a complicated task [33].

\subsubsection{Caveats}

In summary, multiuser MIMO can provide significant gains compared to point-topoint MIMO in a wider range of propagation scenarios. By spatially multiplexing the users in the same time-frequency resources, it can offer theoretically $\min (M, K)$ 
degrees of freedom, provided that specific signal processing techniques are implemented to mitigate the resulting inter-user interference.

MMSE accompanied by SIC for the uplink, and by DPC for the downlink, enable to achieve the sum capacity. However, these interference cancellation techniques require complicated signal processing, and more importantly accurate CSI at both the BS and the users.

CSI acquisition takes place via pilot-based training for which a significant amount of resources (proportional to $K$ in the uplink and to $M$ in the downlink) is reserved for the transmission of uplink and downlink pilots. This (pilot) overhead subtracts resources to the data, hence reducing the performance. Taking the channel estimation into account, multiuser MIMO capacity seldom scales linearly with $\min (M, K)$.

Importantly, multiuser MIMO lacks of scalability: as $M$ and $K$ grow, the complexity of the signal processing and the overhead due to the acquisition of the CSI become unsustainable for the system. For this reason, practical implementations of multiuser MIMO in LTE (and WiFi) include BSs with at most 8 antennas spatially multiplexing no more than 4 users.

\subsection{Massive MIMO}

The solution to the inter-user interference and scalability issues of multiuser MIMO technology has a name: Massive MIMO [33,35].

Giving a rigorous definition of massive MIMO may result too simplistic and perhaps pretentious. A short but full of meaning definition of massive MIMO is given in [33]: "Massive MIMO is a useful and scalable version of multiuser MIMO". The principle that enables to attain this usefulness and scalability is simply having a large (massive) number of antennas at the BS serving, jointly and coherently, a smaller number of users.

\subsubsection{Key Ingredients}

Massive MIMO is like the "pasta alla carbonara", namely a dish apparently simple to cook but actually quite sophisticated. Replacing key ingredients, (e.g., eggs with cream) or not following the original recipe carefully, can change radically the taste and ruin your culinary experience. Hence, it is important to highlight the salient ingredients that make massive MIMO a sensational dish:

- Excessive number of BS antennas over the users. A massive number of antennas at the BS yields a large beamforming and diversity gain. Importantly, signal can be spatially focused towards the users with more precision, enabling an 
aggressive spatial multiplexing. Last but not least, the use of large number of antennas triggers two relevant phenomena deriving from the law of the large numbers known as favorable propagation $[20,33,37,97]$ and channel hardening $[20,33,39,98]$. The former reduces the inter-user interference. The latter reduces the random fluctuations in the effective channel gain seen by the users.

- Time-division duplex (TDD). In TDD mode, the frequency spectrum is shared by the uplink and the downlink which are thereby reciprocal. Hence, the CSI acquired at the BS during the uplink training stage is also valid to construct the precoding vectors.

- Acquisition of CSI only at the BS. Due to the channel hardening, the effective channels seen by the users are nearly deterministic. Therefore, the knowledge of the channel statistics is sufficient for the user to detect the desired signals. Thanks to reciprocity and channel hardening, downlink channel estimation becomes unnecessary [40]. This allows to reduce the estimation overhead.

- Linear signal processing. As a consequence of the favorable propagation, linear signal processing schemes are nearly optimal. This reduces the hardware complexity and the circuit power consumption.

- Predictable accurate performance. By leveraging the law of the large numbers, closed-form expressions for the achievable rate can be derived, and by virtue of the channel hardening these becomes more reliable as the number of BS antennas grows. Moreover, as the fading massive MIMO channel behaves almost as a non-fading channel, coding and modulation schemes that are optimized for the additive white Gaussian noise (AWGN) channel works well in massive MIMO. Hence, the theoretical analysis of massive MIMO is able to predict accurately the performance of practical systems.

- Simplified resource allocation. As a result of the previous point, the mathematical expressions in the massive MIMO literature can be exploited to simplify crucial resource allocation tasks such as power control. Resource allocation does not need to adapt to the small-scale fading variations as the latter vanishes due to the channel hardening. Letting the resource allocation depend only on the large-scale fading quantities gives also room to time-demanding optimization strategies. 


\subsubsection{Favorable Propagation}

A general motivation for massive MIMO is the favorable propagation phenomenon triggered by the large number of antennas at the BS. Favorable propagation brings two main benefits:

1. It mitigates the inter-user interference, thereby increasing the performance;

2. Linear signal processing schemes (e.g., MR, ZF, MMSE) provide achievable rates close to the channel capacity $[27,32,33]$.

\section{Inter-user Interference Mitigation}

As already discussed in Section 2.4, multiuser MIMO performance is limited by the inter-user interference. Let us recall the expression for the sum capacity of the multiple access channel in (7), and consider $K=2$ users and $M$ BS antennas,

$$
\begin{aligned}
C_{\mathrm{ul}}^{\mathrm{sum}} & =\log _{2}\left|\boldsymbol{I}_{M}+\mathrm{SNR} \cdot \boldsymbol{G} \boldsymbol{G}^{\mathrm{H}}\right| \\
& \stackrel{(a)}{=} \log _{2}\left|\boldsymbol{I}_{2}+\mathrm{SNR} \cdot G^{\mathrm{H}} \boldsymbol{G}\right| \\
& =\log _{2}\left|\boldsymbol{I}_{2}+\mathrm{SNR} \cdot\left(\begin{array}{cc}
\left\|\boldsymbol{g}_{1}\right\|^{2} & \boldsymbol{g}_{1}^{\mathrm{H}} \boldsymbol{g}_{2} \\
\boldsymbol{g}_{2}^{\mathrm{H}} \boldsymbol{g}_{1} & \left\|\boldsymbol{g}_{2}\right\|^{2}
\end{array}\right)\right| \\
& =\log _{2}\left[\left(1+\mathrm{SNR} \cdot\left\|\boldsymbol{g}_{1}\right\|^{2}\right)\left(1+\mathrm{SNR} \cdot\left\|\boldsymbol{g}_{2}\right\|^{2}\right)-\mathrm{SNR}^{2} \cdot\left|\boldsymbol{g}_{1}^{\mathrm{H}} \boldsymbol{g}_{2}\right|^{2}\right],
\end{aligned}
$$

where in $(a)$ we have utilized Sylvester's determinant theorem. Figure 3 illustrates the capacity region ${ }^{5}$ of the multiple access channel with $K=2$. The maximum achievable rate for the single user equals the capacity of the corresponding MISO channel with CSI at the transmitter, shown in (1). That is the highest rate that each user achieves if it would have been alone in the network. The height and width of the polygon in Figure 3 are determined by the inequalities

$$
R_{k} \leq \log _{2}\left(1+\mathrm{SNR} \cdot\left\|\boldsymbol{g}_{k}\right\|^{2}\right), \quad k=1,2 .
$$

The pentagonal shape of the capacity region is due to the inter-user interference. More specifically, the length of the upper right side of the pentagon is determined by the sum-rate inequality,

$$
R_{\mathrm{ul}}^{\mathrm{sum}} \leq C_{\mathrm{ul}}^{\mathrm{sum}}
$$

wherein the term $\mathrm{SNR}^{2} \cdot\left|\boldsymbol{g}_{1}^{\mathrm{H}} \boldsymbol{g}_{2}\right|^{2}$ in (10) represents the power of the inter-user interference. The communication is interference-free if the channel vectors, $\boldsymbol{g}_{1}$

\footnotetext{
${ }^{5}$ The rate region is the set of all feasible combinations of the rates which can be achieved simultaneously by all the users. The capacity region is the largest possible rate region [26].
} 


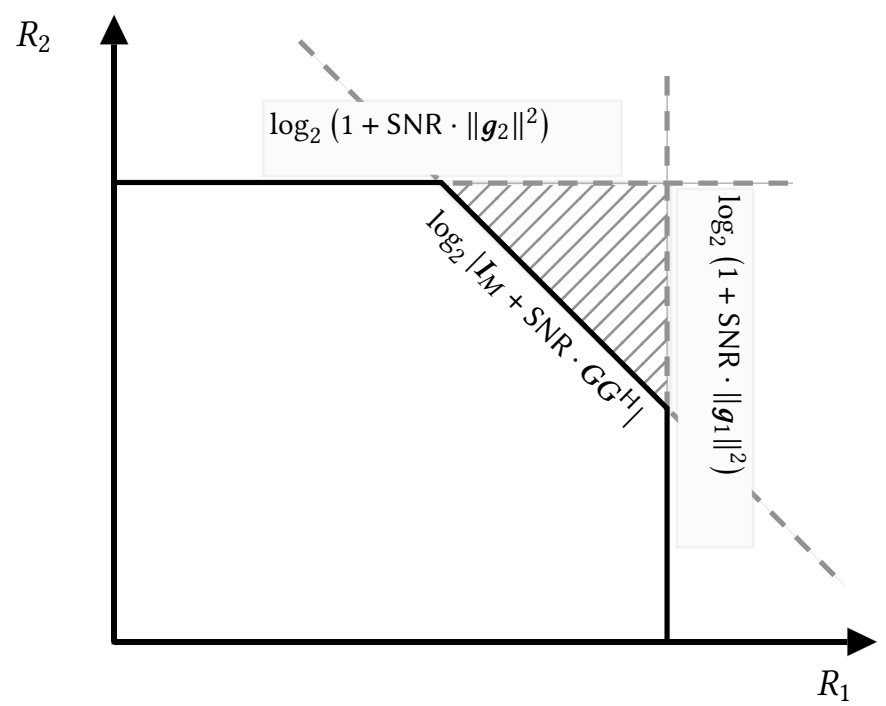

Figure 3: The capacity region of the multiple access channel with $K=2$.

and $\boldsymbol{g}_{2}$, are mutually orthogonal, namely $\boldsymbol{g}_{1}^{\mathrm{H}} \boldsymbol{g}_{2}=0$. The corresponding sum-rate inequality would become

$$
R_{\mathrm{ul}}^{\mathrm{sum}} \leq \log _{2}\left(1+\mathrm{SNR} \cdot\left\|\boldsymbol{g}_{1}\right\|^{2}\right)+\log _{2}\left(1+\mathrm{SNR} \cdot\left\|\boldsymbol{g}_{2}\right\|^{2}\right),
$$

and the capacity region would take on the ideal rectangular shape. Hence, in this case, the sum capacity would be maximized and equal to the summation of the individual user capacities. Both multiplexing and beamforming gains are achieved.

A propagation environment satisfying the orthogonality condition, $\boldsymbol{g}_{1}^{\mathrm{H}} \boldsymbol{g}_{2}=0$, offers favorable propagation. This is the most desirable scenario from the capacity viewpoint as the users do not affect each other when communicating with the BS. However, favorable propagation is quite unlikely to hold in practice but it can be approximately attained. That is where massive MIMO comes in!

Consider the realistic case of fast-fading channels. We say that a propagation environment offers asymptotically favorable propagation if it holds [20,27,33]

$$
\frac{\boldsymbol{g}_{k}^{\mathrm{H}} \boldsymbol{g}_{k^{\prime}}}{\sqrt{\mathbb{E}\left\{\left\|\boldsymbol{g}_{k}\right\|^{2}\right\} \mathbb{E}\left\{\left\|\boldsymbol{g}_{k^{\prime}}\right\|^{2}\right\}}} \stackrel{\text { a. s. }}{\rightarrow} 0, \quad \text { as } M \rightarrow \infty, \quad k \neq k^{\prime},
$$

where the notation "a. s." denotes almost sure convergence. Since $\mathbb{E}\left\{\left\|\boldsymbol{g}_{k}\right\|^{2}\right\}$ is proportional to $M$, asymptotic favorable propagation can be attained by increasing the 
number of BS antennas to infinity. Importantly, a practical favorable propagation can be achieved for $M$ sufficiently large but finite.

For instance, if we assume $\boldsymbol{g}_{k} \sim \mathcal{C N}\left(\mathbf{0}, \boldsymbol{I}_{M}\right)$ (i.i.d. Rayleigh fading) and finite $M$, then $\mathbb{E}\left\{\left\|\boldsymbol{g}_{k}\right\|^{2}\right\}=M$. While, $\operatorname{Var}\left\{\frac{1}{M} \boldsymbol{g}_{k}^{\mathrm{H}} \boldsymbol{g}_{k^{\prime}}\right\}=\frac{1}{M}$ tells us how quickly the normalized inner product of the channel vectors converges to zero. Similarly, the quantity $\frac{1}{M^{2}}\left|\boldsymbol{g}_{k}^{\mathrm{H}} \boldsymbol{g}_{k^{\prime}}\right|^{2} \rightarrow 0$, with speed [37]

$$
\operatorname{Var}\left\{\frac{1}{M^{2}}\left|\boldsymbol{g}_{k}^{\mathrm{H}} \boldsymbol{g}_{k^{\prime}}\right|^{2}\right\}=\frac{M+2}{M^{3}} \approx \frac{1}{M^{2}} .
$$

Hence, the inter-user interference term in (10) vanishes as $M$ grows with rate approximately equal to $1 / M^{2}$.

\section{Outstanding Linear Processing}

To show why linear receivers are nearly optimal, consider a small example concerning the uplink massive MIMO channel with 2 users and $M$ BS antennas:

$$
\boldsymbol{y}=\boldsymbol{g}_{1} x_{1}+\boldsymbol{g}_{2} x_{2}+\boldsymbol{n},
$$

where we let $\boldsymbol{g}_{k} \sim \mathcal{C N}\left(\mathbf{0}, \boldsymbol{I}_{M}\right), \mathbb{E}\left\{\|\boldsymbol{x}\|^{2}\right\}=1$, and $\boldsymbol{n}_{k} \sim \mathcal{C N}\left(\mathbf{0}, \boldsymbol{I}_{M}\right)$. We assume that the BS knows exactly the channel, and uses the receive combining vector $\boldsymbol{a}_{k}=\frac{1}{M} \boldsymbol{g}_{k}(\mathrm{MRC})$ to detect user $k$. Hence, the combined signal for user 1 is given by

$$
\check{y}=\boldsymbol{a}_{1}^{\mathrm{H}} \boldsymbol{g}_{1} x_{1}+\boldsymbol{a}_{1}^{\mathrm{H}} \boldsymbol{g}_{2} x_{2}+\boldsymbol{a}_{1}^{\mathrm{H}} \boldsymbol{n} .
$$

Due to the law of the large numbers the inner products in (13) are approximated by their expected values. Hence, the

- desired signal $x_{1}$ remains since $\frac{1}{M}\left\|\boldsymbol{g}_{1}^{2}\right\| \rightarrow \frac{1}{M} \mathbb{E}\left\{\left\|\boldsymbol{g}_{1}\right\|^{2}\right\}=1$, as $M \rightarrow \infty$,

- interference $x_{2}$ vanishes since $\frac{1}{M} \boldsymbol{g}_{1}^{\mathrm{H}} \boldsymbol{g}_{2} \rightarrow \frac{1}{M} \mathbb{E}\left\{\boldsymbol{g}_{1}^{\mathrm{H}} \boldsymbol{g}_{2}\right\}=0$, as $M \rightarrow \infty$,

- noise $\boldsymbol{n}$ vanishes since $\frac{1}{M} \boldsymbol{g}_{1}^{\mathrm{H}} \boldsymbol{n} \rightarrow \frac{1}{M} \mathbb{E}\left\{\boldsymbol{g}_{1}^{\mathrm{H}} \boldsymbol{n}\right\}=0$, as $M \rightarrow \infty$.

This tells us that interference/noise-free communication can be asymptotically attained as $M \rightarrow \infty$, thereby achieving the ergodic sum capacity

$$
C_{\mathrm{ul}}^{\mathrm{sum}}=\sum_{k=1}^{2} \mathbb{E}\left\{\log _{2}\left(1+\left\|\boldsymbol{g}_{k}\right\|^{2}\right)\right\} .
$$

Figure 4 shows that tens of BS antennas are sufficient to offer favorable propagation in i.i.d. Rayleigh Fading, and drastically reduce the inter-user interference. Certainly, i.i.d. Rayleigh fading constitutes the best-case scenario, but it has been proven, both theoretically and practically, that favorable propagation is supported in most propagation environments $[33,36,99]$. 


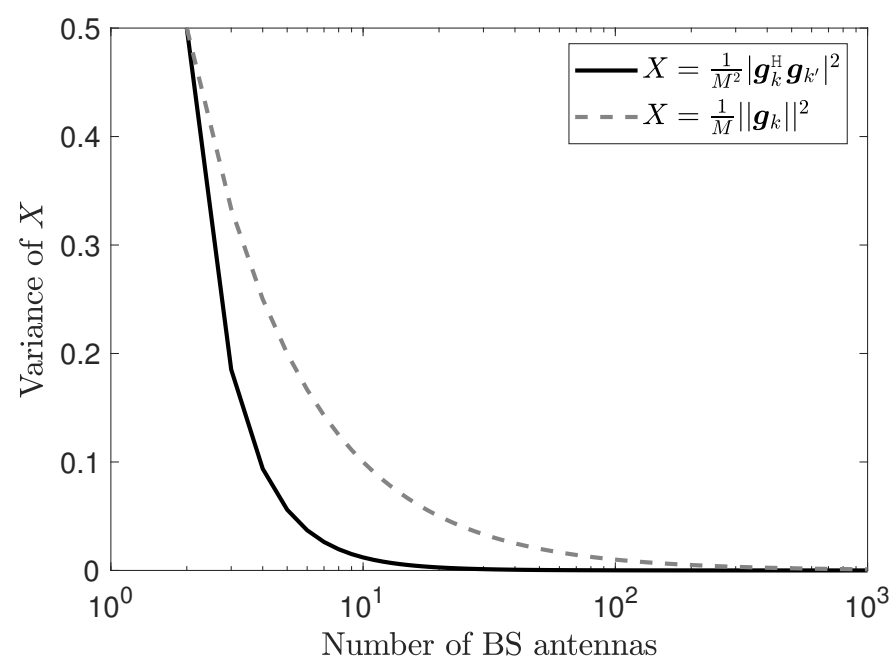

Figure 4: Favorable propagation and channel hardening against number of BS antennas, assuming i.i.d. Rayleigh fading.

\subsubsection{Channel Hardening}

The channel hardening phenomenon approximates a fading massive MIMO channel to a deterministic channel. We say that a channel hardens if the channel gain tends to its mean value as the number of antennas at the BS increases. Mathematically, it is defined as $[20,27,33]$

$$
\frac{\left\|\boldsymbol{g}_{k}\right\|^{2}}{\mathbb{E}\left\{\left\|\boldsymbol{g}_{k}\right\|^{2}\right\}} \stackrel{\text { a.s. }}{\rightarrow} 1, \quad \text { as } M \rightarrow \infty .
$$

Figure 4 shows that, for i.i.d. Rayleigh fading, tens of BS antennas are sufficient for the user to experience channel hardening, although the speed of the convergence,

$$
\operatorname{Var}\left\{\frac{1}{M}\left\|\boldsymbol{g}_{k}\right\|^{2}\right\}=\frac{1}{M}
$$

is slower than that of favorable propagation in (12). This suggests that channel hardening is more sensitive to the propagation environment than favorable propagation, and thereby this property might be less pronounced in practical massive MIMO channels, as recently demonstrated by measurements campaigns [39]. It is worth to emphasize that channel hardening is not necessary for massive MIMO to work [33]. However, as already introduced in Section 2.5.1, it facilitates signal processing and the ergodic capacity analysis. Specifically, channel hardening allows to derive reliable capacity lower bounds in closed form, namely capacity expressions 
that depend only on deterministic quantities. These lower bounds become tighter to the actual capacity as we increase the number of the BS antennas.

\subsubsection{TDD Operation}

Before discussing how TDD works and why it is advocated in massive MIMO, let us introduce a typical assumption which is reasonable in most of mobile communication systems with time-slotted transmission: the block-fading channel model.

\section{Block Fading}

In the block-fading model, the channel varies over time and frequency but the time-frequency resources are divided into blocks, referred to as coherence blocks (or coherence intervals), wherein the channel between any antenna-user pair is time-invariant and frequency flat.

Specifically, a coherence block consists of $\tau_{\mathrm{c}}=T_{\mathrm{c}} B_{\mathrm{c}}$ samples (or channel uses), where $T_{\mathrm{c}}$ is the coherence time and $B_{\mathrm{c}}$ is the coherence bandwidth, see Figure 5 . The coherence time is defined as the time interval over which the channel may be considered time-invariant. It is proportional to the wavelength and depends on user's speed $[20,26,33]$. The coherence bandwidth is defined as the frequency interval over which the channel may be considered frequency flat. It is inversely proportional to the delay spread of the channel, namely the time difference between the shortest and the longest path of the considered multipath model $[20,26,33]$.

The dimension of a coherence block matches that of the user with shortest coherence time/bandwidth. In $\tau_{\mathrm{c}}$ samples, each coherence block accommodates CSI acquisition and data transmission.

In summary, the block-fading model is just an instance of fast fading channel, where we have one random channel realization per coherence block. Hence, we look at the ergodic capacity metric as we have long codewords and code over many coherence blocks. In the context of multicarrier communication systems (e.g., LTE, NR) a coherence block can be seen as a block encompassing one or a set of subcarriers which spans over one or multiple time-slots.

So far, for the sake of simplicity, we have considered either deterministic or slow-fading channels. For the rest of this dissertation we assume block-fading channels.

\section{Why TDD?}

In TDD mode, uplink and downlink communications share the same spectrum but at different times, as shown in Figure 5. As a result, uplink and downlink channels 


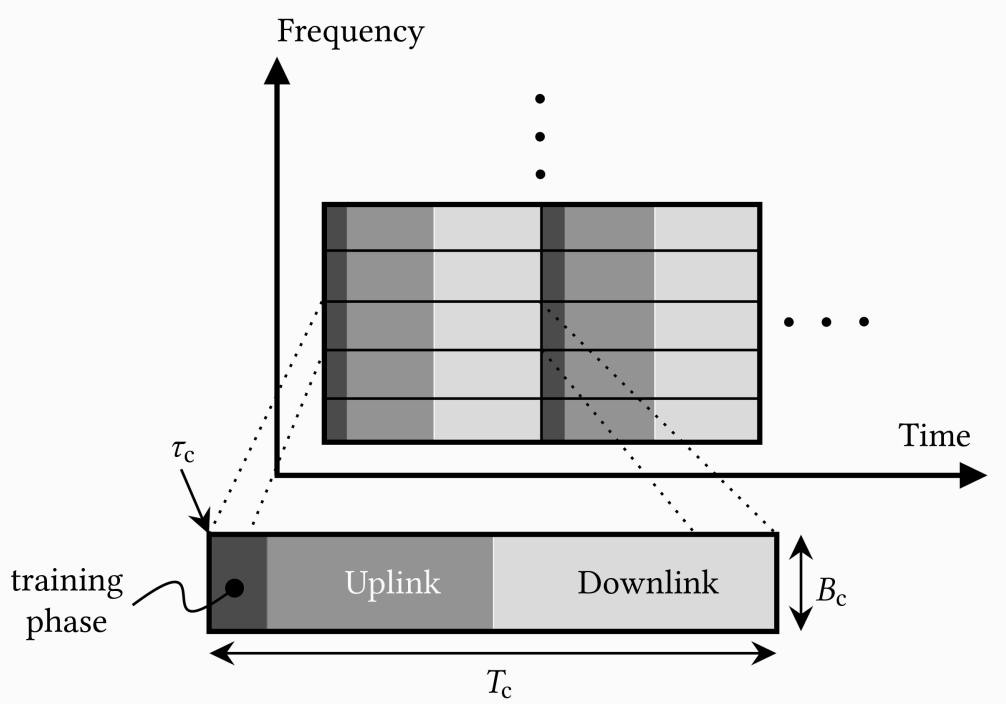

Figure 5: An illustration of the time-frequency resources divided into coherence blocks with focus on the TDD frame structure.

are reciprocal, namely $\boldsymbol{g}_{\mathrm{ul}}=\boldsymbol{g}_{\mathrm{dl}}^{\mathrm{T}}{ }^{6}$

By leveraging the channel reciprocity, CSI acquisition can be carried out only in one way, from the users to the BS. The BS uses the uplink channel estimates to perform precoding while the users can efficiently decode the data by only using the statistical knowledge of the channel, thanks to the channel hardening.

For example, consider $K$ users, $M$ BS antennas and that channel estimation takes place via orthogonal pilot transmission. The amount of estimation resources needed in TDD massive MIMO is simply $K$ samples, required to accommodate $K$ pilots in the uplink training phase. Hence, channel estimation is independent of $M$.

A conventional downlink training would need $M$ pilots plus $M$ CSI coefficients fed back by each user to the BS. Since the length of the coherence interval is finite and $M$ is large, the penalty of the channel estimation overhead on the performance becomes too heavy and the communication inefficient. In some circumstances where the coherence interval is not sufficiently long (e.g., moderate/high user mobility), fitting such a large amount of estimation resources in a coherence interval is even physically impossible.

In summary, TDD operation is key in massive MIMO to preserve the scalability and to keep the amount of resources needed for the channel estimation small.

\footnotetext{
${ }^{6}$ Although a calibration is needed to compensate the mismatches between the transmit and receive radio frequency $(\mathrm{RF})$ chains, reciprocity to the required accuracy can be achieved in practice using off-the-shelf methods [100]. For this reason, we assume perfect reciprocity throughout this thesis.
} 


\section{Chapter 3}

\section{Distributed MIMO Systems}

This chapter gives an overview on distributed MIMO systems, discussing pros and cons of having multiple cooperating antennas geographically spread out rather than packed into a BS. Such systems are attractive from the performance viewpoint as they can resolve the inter-cell interference and offer an extraordinary diversity gain. However, these advantages come at the cost of higher implementation complexity. Cell-free massive MIMO is the latest attempt in providing a successful solution for distributed MIMO architectures. In this chapter, as well as in the rest of this thesis, we describe the enormous potential of this promising concept.

\subsection{Taxonomy}

The ambition of this section is not to draw a sharp line between different implementations of distributed MIMO systems, but rather to relate similar solutions proposed in the last two decades under different names to each other, and finally collocate cell-free massive MIMO in the distributed MIMO literature.

\subsubsection{Network MIMO}

The term "network MIMO" embodies the principle that multiple cells organically cooperate to act as a single network and serve all the users in its joint coverage area. Hence, broadly speaking, network MIMO is a synonym of distributed MIMO, and includes all its subsequent variants. Strictly speaking, the concept of network MIMO arose in the early 2000s $[60,61,101]$ mainly in the form of cooperative MIMO BSs serving all the users in their range of influence by using multiuser MIMO processing techniques (e.g., DPC), and under the assumption of perfect CSI knowledge. The BSs are grouped into disjoint static cooperation clusters. Each cluster is served by a central processing unit (CPU) which is responsible 
of the BS cooperation, collecting the CSI from all the BSs and performing joint precoding/combining in a centralized fashion. A fronthaul network connects the CPU to all the BSs in its cluster.

The great ability of network MIMO in mitigating the inter-cell interference and providing excellent SE has been confirmed in several studies [62-65], under realistic operational conditions. In particular, [64] introduces the terminology "network MIMO", while [65] brings into the analysis, for the first time, the effects of channel estimation error at the BS and the cost of the estimation, assuming centralized zeroforcing beamforming. Interestingly, [65] also proposes an overlapped clustering system architecture where adjacent BSs are grouped into clusters, and the clustering patterns are shifted versions of each other, overlapped in space, such that there are no cluster-edge users.

\subsubsection{Cooperative Multi-cell MIMO}

Cooperative multi-cell MIMO is a terminology used, for instance, in $[68,102$, 103] as a synonym of network MIMO. However, $[68,103]$ point out the practical limitations of "conventional" network MIMO which relies on fully centralized signal processing, and requires the exchange of instantaneous CSI among the BSs. Hence, in cooperative multi-cell MIMO, distributed precoding/combining with partial (or no) CSI sharing is advocated in order to reduce the requirements on fronthaul capacity and computational complexity.

Importantly, $[68,103]$ address the important issue of scalability by envisioning practical implementations of cooperative networks based on local channel estimation, distributed signal processing and limited cooperation via dynamic clustering [102,104,105]. (These are salient features in cell-free massive MIMO.) In particular, the main idea of dynamic clustering is that each user is served by a tailored cluster consisting of near-by BSs. This allows to confine the cooperation within a handful of adjacent BSs, thereby alleviating the fronthauling load. Moreover, dynamic clustering reduces the inter-cluster interference compared to a static clustering as a user is more unlikely to stand at the edge of a cluster.

Although a step forward to a better understanding of the information-theoretic capacity of cooperative multi-cell MIMO has been done in $[68,103,105]$, yet channel uncertainty was not been adequately treated as perfect CSI was often assumed in these works.

\subsubsection{CoMP}

Coordinated MultiPoint (CoMP) is essentially the commercial name of network MIMO in LTE-Advanced. CoMP, included in LTE Release 11 [106], implements 
several network/cooperative MIMO techniques for inter-cell interference coordination [70,71] and cooperation clustering [72]. The cooperation and the interference mitigation framework were designed for macrocell networks. With the advent of 5G, different visions for CoMP systems have been proposed [74], bringing the inter-cell interference coordination at the small cell level, and leveraging co-located massive MIMO to reduce the out-of-cluster interference [107].

\subsubsection{C-RAN}

C-RAN (Cloud-Radio Access Network) is a cloud-computing based architecture for radio access networks [108]. In C-RAN, the baseband processing is moved to a central processor, referred to as BaseBand Unit (BBU), which is connected to several Remote Radio Units (RRU) via optical fiber fronthaul. The RRU is an access point (AP) that simply acts as a device forwarding signals between the BBU and the users. All the processing complexity is at the BBU.

C-RAN offers a better support for CoMP implementations in 5G, as the optical fiber fronthaul, with its high capacity and low latency, is more suitable to support multi-cell cooperation than the traditional LTE interfaces.

However, full centralization is critical in C-RAN and gives rise to the aforementioned scalability issues [68] in ultra-dense networks. In this regard, C-RAN architectures implementing dynamic cooperation clustering have been proposed in $[109,110]$ : each user is surrounded by selected serving RRU such that it "falls" in the center of the created cluster. This is also known as user-centric ${ }^{1}$ architecture, and it succeeds when the network is sufficiently dense and connected.

C-RAN architecture might be used to implement a cell-free massive MIMO system, although some baseband processing capability is required at the APs in order to de-centralize some tasks such as channel estimation and distributed precoding/combining.

\subsubsection{Related Concepts}

Distributed Antenna Systems (DAS) and virtual MIMO are terms equivalent to network MIMO. Both describe a system where distributed antennas, coordinated by a central processor, virtually act as a macroscopic multiuser MIMO system. Hence, MIMO information theory can be reused to evaluate the achievable ergodic capacity (see e.g., [66] for DAS and [69] for virtual MIMO).

In [76] the terminology Extremely Large Aperture Array (ELAA) has been used to describe any system consisting of distributed BS antennas that jointly

\footnotetext{
${ }^{1}$ Conventional cellular systems are network-centric: the users surround the macro BSs, which are in the cell-center.
} 
and coherently serve distributed users. The ELAA terminology emphasizes the large dimension of the distributed array where the antenna separation is at the order of meters, compared to the conventional compact arrays where the antenna separation is in the order of the wavelength (in the range from one decimeter down to few millimeters). All the network-MIMO-based systems are covered by the definition of ELAA as well as the so called very large aperture massive MIMO systems [111] and the extra-large-scale massive MIMO systems [112,113]. The spatial non-stationary field properties [114] over these extremely large arrays have been studied in [111-113]. Basically, given an ELAA with $M$ antennas, each user sees only a portion of the ELAA, that is a number of antennas significantly smaller than $M$. Changing perspective, different portions of the ELAA observe either the same channels with varying strength or totally different channels towards each user. In this regard, subarray-based architectures have been proposed in [112,115] to reduce the complexity of the centralized baseband processing by exploiting the spatial non-stationary properties of the channels.

\subsection{Cell-Free Massive MIMO}

Once we had a closer look at the state-of-the-art on distributed MIMO systems, it is much easier to describe what cell-free massive MIMO [56-58] is, or rather what is not: cell-free massive MIMO is not a novel concept but is certainly an evolution of network MIMO. Just like massive MIMO is a scalable version of multiuser MIMO, cell-free massive MIMO is a scalable embodiment of network MIMO [58].

\subsubsection{Features}

Cell-free massive MIMO combines the following elements:

1. Massive MIMO baseline operation. In cell-free massive MIMO, the APs and the CPUs operate as a massive MIMO single cell. Hence, it inherits all the outstanding features of co-located massive MIMO, and embodies all the ingredients described in Section 2.5.1.

2. Extraordinary Macro-diversity. As a result of the distributed topology and the ultra densification, cell-free massive MIMO can offer unprecedented levels of macro-diversity gain, which entails a more reliable communication link and a stronger channel gain. Macro-diversity is discussed more in detail in Paper A.

3. User-centric implementation $[116,117]$, which is crucial to mitigate the intercluster interference (as described in Section 3.1), and to preserve the system 
scalability. The scalability of cell-free massive MIMO is the main theme of Paper B.

4. Uniformly great service [56]. As a result of the outstanding macro-diversity and the user-centric approach, cell-free massive MIMO guarantees by nature uniformly excellent QoS to all the users. This aspect is emphasized in Paper A. Interestingly, egalitarian power control strategies such as max-min fairness works better in cell-free massive MIMO than in co-located massive MIMO, wherein the cell-edge users jeopardize the performance of the cell-center users. $^{2}$

\subsubsection{Challenges and Key Enablers}

Cell-free massive MIMO inherits pros and cons of network MIMO. Joint coherent processing among the APs might be computationally demanding, and in turn might require high-capacity fronthaul network, significant amounts of signalling overhead $^{3}$ and exchange of either instantaneous or statistical CSI.

First studies on cell-free massive MIMO [56-58] aimed to give a first sense on the performance of this promising concept, although under unpractical assumptions: all the distributed APs, coordinated by only one CPU over an infinite-capacity fronthaul, jointly coherently serve all the users in a infinitely ${ }^{4}$ wide network. Hence, a cell-free massive MIMO system is modeled as an infinitely wide massive MIMO single cell. Hereafter, this system model is referred to as the canonical form of cell-free massive MIMO and is presented in detail in the next section.

If, on the one hand, the canonical form of cell-free massive MIMO is scalable thanks to a local one-way CSI acquisition and distributed linear precoding/combining schemes, then, on the other hand, the notion that the "whole world" constitutes a network is unpractical and unscalable. A practical, scalable implementation should take into account that the CPU and the fronthaul constitute the bottleneck of the architecture, and thereby:

- Data sharing and resource allocation tasks (e.g., power control, pilot assign-

\footnotetext{
${ }^{2}$ The soundness of max-min fairness in cell-free massive MIMO led us to mainly focus on this power control strategy in this dissertation. Importantly, max-min fairness power control can be straightforwardly extended to weighted max-min fairness by including weights which account for user priority and can adjust the rate target according to each user need.

${ }^{3}$ With the term signalling overhead, we mean all the non-data information exchanged over the fronthaul between CPUs and APs. For instance, information concerning the precoding/combining, power control, synchronization, pilot assignment, etc. CSI can be also included in the signalling overhead.

${ }^{4} \mathrm{~A}$ wrap-around technique over a nominal area is employed to simulate a single cell without boundaries, and thereby erase the inter-cell interference.
} 
ment, etc.) must be confined within few clusters of APs, in order to limit the computational complexity at the $\mathrm{CPU}$ and the signalling overhead;

- Fully centralized precoding and combining schemes must be avoided, in order to overcome the need of instantaneous CSI at the CPU;

- Any attempt of improving the performance through "non-conventional" resource allocation and/or decoding schemes involving the CPU should solely rely on large-scale fading quantities, in order to limit the amount of information exchanged over the fronthaul as well as the frequency of these exchanges;

- Symbol-level synchronization is crucial to enable joint coherent transmission, and clustering is necessary to minimize delay and synchronization errors;

- Signal quantization techniques should be developed to cope with limited fronthaul and backhaul ${ }^{5}$ capacity.

Over the last five years, great efforts have been spent to achieve these desiderata and make cell-free massive MIMO practical. This dissertation and the relevant related works cited herein are good examples of this. These studies are sufficient to strengthen the claim that cell-free massive MIMO is a scalable and practical version of network MIMO. However, cell-free massive MIMO has at least two aces up its sleeve to be acknowledged as the ultimate embodiment of network MIMO:

1. The potential marriage between the mature massive MIMO technology and mmWave, not only moving up in frequency the operating regime to further increase the SE, but also replacing optical fiber cables with mmWave links to make the fronthaul flexible and less costly;

2. The emerging low-cost and flexible solutions for cell-free network deployments, such as $p$ Cell $[118,119]$ (a product resembling a C-RAN architecture) and the radio stripes $[80,120]$ (a concept wherein the antennas are serially located in/on stripes and the signal processing is carried out sequentially). The latter is discussed in Paper A.

\subsubsection{The Canonical Form: System Model and Performance}

For the sake of completeness, we next briefly report the system model of cell-free massive MIMO in its canonical form, and the achievable SE expressions in closed

\footnotetext{
${ }^{5}$ We refer to as the fronthaul network the links between APs and CPUs, while the backhaul consists of the links between CPUs.
} 
form derived in [58]. The scope of this section is to provide the reader with the analytical framework used in Paper A and Paper B wherein its detailed description was, however, omitted.

Consider a cell-free massive MIMO system wherein $M$ single-antenna APs simultaneously serve $K$ single-antenna users in the same time-frequency resources and operating in TDD mode. The APs are connected to a CPU via an infinitecapacity fronthaul network.

CSI acquisition occurs only at the APs. MMSE channel estimation is carried out locally at each AP via pilot-based uplink (UL) training, provided that the channel distribution (statistical CSI) is known a priori. By exploiting the (perfect) channel reciprocity, the estimates are then used in the downlink (DL) to perform conjugate beamforming. The users decode the data relying only on the statistical CSI. In the UL, each AP sends soft symbols (i.e., after matched filtering) to the CPU. Hence, the APs do not need to share CSI as the processing is fully distributed.

The channel between user $k$ and AP $m$ is denoted by $g_{m k}=\sqrt{\beta_{m k}} h_{m k}$, where $\beta_{m k}$ is the large-scale fading coefficient (which accounts for path loss and shadowing), and $h_{m k} \sim \mathcal{C N}(0,1)$ is the small-scale fading coefficient (i.i.d. Rayleigh fading). The block fading channel model described in Section 2.5.4 is assumed.

The TDD coherence interval is $\tau_{\mathrm{c}}$ samples long and consists of three phases: UL training, UL data transmission and DL data transmission.

\section{Uplink Training}

During the UL training, all the $K$ users synchronously send their own pilot, that is a pre-assigned sequence known at both the ends of the link. The UL training length corresponds to the length of the pilot and is denoted by $\tau_{\mathrm{p}} \leq \tau_{\mathrm{c}} / 2$ (according to the rule-of-thumb in [35]).

Let $\sqrt{\tau_{\mathrm{p}}} \boldsymbol{\phi}_{k} \in \mathbb{C}^{\tau_{\mathrm{p}}}$ be the pilot sequence assigned to user $k$, where $\boldsymbol{\phi}_{k}$ has unitary norm. At AP $m$ the superposition of the pilots from all the users is given by

$$
\boldsymbol{y}_{\mathrm{p}, m}=\sqrt{\tau_{\mathrm{p}} \rho_{\mathrm{p}}} \sum_{k=1}^{K} g_{m k} \boldsymbol{\phi}_{k}+\boldsymbol{\omega}_{\mathrm{p}, m}
$$

where $\rho_{\mathrm{p}}$ is the pilot symbol SNR and $\omega_{\mathrm{p}, m}$ is additive noise whose elements are i.i.d. $\mathcal{C N}(0,1)$ random variables. Pilots are transmitted with full power.

In order to separate the $K$ streams, AP $m$ performs de-spreading by correlating the received signal with each pilot sequence. Concerning user $k$, we have

$$
\check{y}_{\mathrm{p}, m k}=\boldsymbol{\phi}_{k}^{\mathrm{H}} \boldsymbol{y}_{\mathrm{p}, m}=\sqrt{\tau_{\mathrm{p}} \rho_{\mathrm{p}}} g_{m k}+\sqrt{\tau_{\mathrm{p}} \rho_{\mathrm{p}}} \sum_{k^{\prime} \neq k}^{K} g_{m k^{\prime}} \boldsymbol{\phi}_{k}^{\mathrm{H}} \boldsymbol{\phi}_{k^{\prime}}+\boldsymbol{\phi}_{k}^{\mathrm{H}} \boldsymbol{\omega}_{\mathrm{p}, m},
$$


where the second term is the interference caused by all the users $k^{\prime} \neq k$ whose pilots are not orthogonal to the pilot of user $k$, and referred to as pilot contamination.

This statistic is used to compute the MMSE estimate of $g_{m k}$ given $\check{y}_{\mathrm{p}, m k}$ :

$$
\hat{g}_{m k}=\frac{\mathbb{E}\left\{\check{y}_{\mathrm{p}, m k}^{*} g_{m k}\right\}}{\mathbb{E}\left\{\left|\check{y}_{\mathrm{p}, m k}\right|^{2}\right\}} \check{y}_{\mathrm{p}, m k}=\frac{\sqrt{\tau_{\mathrm{p}} \rho_{\mathrm{p}}} \beta_{m k}}{\tau_{\mathrm{p}} \rho_{\mathrm{p}} \sum_{k^{\prime}=1}^{K} \beta_{m k^{\prime}}\left|\boldsymbol{\phi}_{k}^{\mathrm{H}} \boldsymbol{\phi}_{k^{\prime}}\right|^{2}+1} \check{y}_{\mathrm{p}, m k} .
$$

Hence, in presence of pilot contamination, $\hat{g}_{m k}$ is dependent on $\left\{\hat{g}_{m k^{\prime}}\right\}$ and degraded. The channel estimate is distributed as $\hat{g}_{m k} \sim \mathcal{C N}\left(0, \gamma_{m k}\right)$, where

$$
\gamma_{m k}=\frac{\tau_{\mathrm{p}} \rho_{\mathrm{p}} \beta_{m k}^{2}}{\tau_{\mathrm{p}} \rho_{\mathrm{p}} \sum_{k^{\prime}=1}^{K} \beta_{m k^{\prime}}\left|\boldsymbol{\phi}_{k}^{\mathrm{H}} \boldsymbol{\phi}_{k^{\prime}}\right|^{2}+1} .
$$

\section{Downlink Data Transmission}

Let $q_{k}$ be the data symbol intended for user $k$, and $\mathbb{E}\left\{\left|q_{k}\right|^{2}\right\}=1$. $\left\{q_{k}\right\}$ are mutually independent, and independent of noise and channel coefficients. AP $m$ serves the $K$ users by precoding the data symbol with the conjugate of the related channel estimate (conjugate beamforming):

$$
x_{m}=\sqrt{\rho_{\mathrm{d}}} \sum_{k=1}^{K} \sqrt{\eta_{m k}} \hat{g}_{m k}^{*} q_{k},
$$

where $\rho_{\mathrm{d}}$ is the data symbol SNR. ${ }^{6}$ The data transmission is subject to a per-AP average power constraint, $\mathbb{E}\left\{\left|x_{m}\right|^{2}\right\} \leq \rho_{\mathrm{d}}$, which is equivalent to

$$
\sum_{k=1}^{K} \eta_{m k} \gamma_{m k} \leq 1, \quad \text { for all } m
$$

The coefficient $\eta_{m k}$ adjusts the transmit power over the link $m$-to- $k$ subject to the power constraint. User $k$ decodes $q_{k}$ from the received symbol $r_{\mathrm{d}, k}$, given by

$$
r_{\mathrm{d}, k}=\sum_{m=1}^{M} g_{m k} x_{m}+\omega_{\mathrm{d}, k}=\sqrt{\rho_{\mathrm{d}}} \sum_{m=1}^{M} \sum_{k^{\prime}=1}^{K} \sqrt{\eta_{m k^{\prime}}} g_{m k} \hat{g}_{m k^{\prime}}^{*} q_{k^{\prime}}+\omega_{\mathrm{d}, k},
$$

where $\omega_{\mathrm{d}, k} \sim \mathcal{C N}(0,1)$ is additive noise.

\footnotetext{
${ }^{6} \rho_{\mathrm{d}}$ is equal to the transmit power divided by the noise power. This normalization is just a notational convention with no physical meaning as the noise is at the receiver.
} 


\section{Uplink Data Transmission}

In the uplink, each user sends its data symbol $s_{k}$ to all the APs, where $\mathbb{E}\left\{\left|s_{k}\right|^{2}\right\}=1$. $\left\{s_{k}\right\}$ are mutually independent, and independent of noise and channel coefficients. The maximum (normalized) transmit power is denoted by $\rho_{\mathrm{u}}$. User $k$ transmits a fraction of $\rho_{\mathrm{u}}$, determined by the power control coefficient $0 \leq \eta_{k} \leq 1$. The received signal at the $m$ th $\mathrm{AP}$ is

$$
y_{\mathrm{u}, m}=\sqrt{\rho_{\mathrm{u}}} \sum_{k=1}^{K} g_{m k} \sqrt{\eta_{k}} s_{k}+\omega_{\mathrm{u}, m},
$$

where $\omega_{\mathrm{u}, m} \sim \mathcal{C N}(0,1)$ is additive noise. Then, AP $m$ multiplies $y_{\mathrm{u}, m}$ by $\hat{g}_{m k}^{*}$ (matched filtering) and sends this soft symbol to the CPU. The CPU collects the contributions from all the APs and detects $s_{k}$ from

$$
r_{\mathrm{u}, k}=\sum_{m=1}^{M} \hat{g}_{m k}^{*} y_{\mathrm{u}, m}=\sqrt{\rho_{\mathrm{u}}} \sum_{k^{\prime}=1}^{K} \sum_{m=1}^{M} \sqrt{\eta_{k^{\prime}}} \hat{g}_{m k}^{*} g_{m k^{\prime}} s_{k^{\prime}}+\sum_{m=1}^{M} \hat{g}_{m k}^{*} \omega_{\mathrm{u}, m} .
$$

\section{Capacity Lower Bounds}

The capacity lower bounds derived in [58], and reported in this section, account for the receiver's lack of CSI knowledge, channel estimation error and pilot contamination. An achievable DL SE can be obtained by using the capacity bounding technique in [33, Section 2.3.4], [20,121] known as hardening bound, as this bound is tight to the true capacity if channel hardening holds.

The received data symbol at user $k, r_{\mathrm{d}, k}$, can be rewritten as

$$
r_{\mathrm{d}, k}=\mathrm{DS}_{k} \cdot q_{k}+\mathrm{BU}_{k} \cdot q_{k}+\sum_{k^{\prime} \neq k}^{K} \mathrm{UI}_{k k^{\prime}} \cdot q_{k^{\prime}}+\omega_{\mathrm{d}, k},
$$

where

$$
\begin{aligned}
& \mathrm{DS}_{k} \triangleq \sqrt{\rho_{\mathrm{d}}} \sum_{m=1}^{M} \sqrt{\eta_{m k}} \mathbb{E}\left\{g_{m k} \hat{g}_{m k}^{*}\right\}, \\
& \mathrm{BU}_{k} \triangleq \sqrt{\rho_{\mathrm{d}}} \sum_{m=1}^{M}\left(\sqrt{\eta_{m k}} g_{m k} \hat{g}_{m k}^{*}-\sqrt{\eta_{m k}} \mathbb{E}\left\{g_{m k} \hat{g}_{m k}^{*}\right\}\right), \\
& \mathrm{UI}_{k k^{\prime}} \triangleq \sqrt{\rho_{\mathrm{d}}} \sum_{m=1}^{M} \sqrt{\eta_{m k}} g_{m k} \hat{g}_{m k^{\prime}}^{*},
\end{aligned}
$$


represent the desired signal, the beamforming gain uncertainty (BU), and the interuser interference, respectively. The BU is the error due to the fact that the users employ the expected effective channel gain for data detection, rather than the actual one. The more the channel hardens, the smaller this error is.

By treating the sum of the second, third, and fourth terms in (16) as uncorrelated effective noise, an achievable DL SE of user $k$ is given by

$$
\mathrm{SE}_{\mathrm{d}, k}=\xi_{\mathrm{d}}\left(1-\frac{\tau_{\mathrm{p}}}{\tau_{\mathrm{c}}}\right) \log _{2}\left(1+\mathrm{SINR}_{\mathrm{d}, k}\right), \quad[\mathrm{bit} / \mathrm{s} / \mathrm{Hz}],
$$

where $0<\xi_{\mathrm{d}}<1$ is the fraction of data samples spent for the DL transmission, the pre-log factor $1-\tau_{\mathrm{p}} / \tau_{\mathrm{c}}$ accounts for the pilot overhead, and

$$
\mathrm{SINR}_{\mathrm{d}, k}=\frac{\left|\mathrm{DS}_{k}\right|^{2}}{\mathbb{E}\left\{\left|\mathrm{BU}_{k}\right|^{2}\right\}+\sum_{k^{\prime} \neq k}^{K} \mathbb{E}\left\{\left|\mathrm{UI}_{k k^{\prime}}\right|^{2}\right\}+1} .
$$

Computing the expectations with respect to the channel realizations, the corresponding closed-form expression for the effective SINR is given by [58]

$$
\operatorname{SINR}_{\mathrm{d}, k}=\frac{\rho_{\mathrm{d}}\left(\sum_{m=1}^{M} \sqrt{\eta_{m k}} \gamma_{m k}\right)^{2}}{\rho_{\mathrm{d}} \sum_{k^{\prime} \neq k}^{K}\left(\sum_{m=1}^{M} \sqrt{\eta_{m k^{\prime}}} \gamma_{m k^{\prime}} \frac{\beta_{m k}}{\beta_{m k^{\prime}}}\right)^{2}\left|\boldsymbol{\phi}_{k^{\prime}}^{\mathrm{H}} \boldsymbol{\phi}_{k}\right|^{2}+\rho_{\mathrm{d}} \sum_{k^{\prime}=1}^{K} \sum_{m=1}^{M} \eta_{m k^{\prime}} \gamma_{m k^{\prime}} \beta_{m k}+1} .
$$

Similarly, an achievable UL SE can be obtained by using the so called use-andforget bound [33, Section 2.3.4] as the channel estimates are used at the APs to post-process the received symbols, which are then sent to the CPU where only the statistical CSI is available to perform data detection. A closed-form expression for an achievable UL SE is given by [58]

$$
\mathrm{SE}_{\mathrm{u}, k}=\xi_{\mathrm{u}}\left(1-\frac{\tau_{\mathrm{p}}}{\tau_{\mathrm{c}}}\right) \log _{2}\left(1+\mathrm{SINR}_{\mathrm{u}, k}\right), \quad[\mathrm{bit} / \mathrm{s} / \mathrm{Hz}],
$$

where $\xi_{\mathrm{u}}=1-\xi_{\mathrm{d}}$ is the fraction of data samples spent for the UL transmission, and

$$
\operatorname{SINR}_{\mathrm{u}, k}=\frac{\rho_{\mathrm{u}} \eta_{k}\left(\sum_{m=1}^{M} \gamma_{m k}\right)^{2}}{\rho_{\mathrm{u}} \sum_{k^{\prime} \neq k}^{K} \eta_{k^{\prime}}\left(\sum_{m=1}^{M} \gamma_{m k} \frac{\beta_{m k^{\prime}}}{\beta_{m k}}\right)^{2}\left|\boldsymbol{\phi}_{k}^{\mathrm{H}} \boldsymbol{\phi}_{k^{\prime}}\right|^{2}+\rho_{\mathrm{u}} \sum_{k^{\prime}=1}^{K} \eta_{k^{\prime}} \sum_{m=1}^{M} \gamma_{m k} \beta_{m k^{\prime}}+\sum_{m=1}^{M} \gamma_{m k}} .
$$


Generalizing the hardening bound for multi-antenna APs, any precoding scheme and any channel model, the expression for the effective DL SINR is given by

$$
\operatorname{SINR}_{\mathrm{d}, k}=\frac{\rho_{\mathrm{d}}\left|\sum_{m=1}^{M} \sqrt{\eta_{m k}} \mathbb{E}\left\{\boldsymbol{g}_{m k}^{\mathrm{H}} \boldsymbol{w}_{m k}\right\}\right|^{2}}{\rho_{\mathrm{d}} \sum_{k^{\prime}=1}^{K} \mathbb{E}\left\{\left|\sum_{m=1}^{M} \sqrt{\eta_{m k^{\prime}}} \boldsymbol{g}_{m k}^{\mathrm{H}} \boldsymbol{w}_{m k^{\prime}}\right|^{2}\right\}-\rho_{\mathrm{d}}\left|\sum_{m=1}^{M} \sqrt{\eta_{m k}} \mathbb{E}\left\{\boldsymbol{g}_{m k}^{\mathrm{H}} \boldsymbol{w}_{m k}\right\}\right|^{2}+1},
$$

where $\boldsymbol{g}_{m k} \in \mathbb{C}^{N}$ is the channel vector assuming APs equipped with $N$ antennas. While, $\boldsymbol{w}_{m k} \in \mathbb{C}^{N}$ is the precoding vector used by AP $m$ towards user $k$ with $\mathbb{E}\left\{\left\|\boldsymbol{w}_{m k}\right\|^{2}\right\}=1$. This bound is used in most of the works of this thesis. 



\section{Chapter 4}

\section{Scope and Related Works}

The scope of this thesis is to look at three aspects of cell-free massive MIMO: $(i)$ scalability, (ii) signal processing and (iii) power control.

Concerning the signal processing aspects, we focus on designing fully distributed and scalable precoding schemes that can improve the spectral efficiency (Paper C, Paper D, Paper E). Our "modus operandi" is to assume independent Rayleigh fading channels in order to derive closed-form expressions for an achievable downlink SE, and thereby discuss insightful results. These expressions account for channel estimation errors, pilot contamination and lack of CSI at the user (except for Paper D). Importantly, these expressions are then employed to devise optimal power control strategies.

Scalability aspects of cell-free massive MIMO have been also addressed in [122124] which propose scalable algorithms for joint initial access, pilot assignment, and cluster formation. The user-centric implementation is extensively discussed in [117], while [125] identifies some issues of decentralizing the co-processing over multiple CPUs in a similar way to Paper B but considering zero-forcing precoding. A user-centric seamless architecture is presented in [126] and dubbed fog massive MIMO. It is essentially an instance of cell-free massive MIMO where clustering is carried out on the fly by the APs which autonomously decide whether serving a user based on local pilot observations. In this architecture, the physical layer processing is fully localized at the APs while geographic routing with packet replication is performed at the network layer.

\section{Power is Nothing without Control}

As already mentioned in the previous chapter, we mainly look at max-min fairness (MMF) power control for two reasons: firstly, because MMF fits perfectly into cellfree massive MIMO which offers uniformly great service per se; secondly, because 
its framework can easily be extended to more general power control strategies by introducing weights that scale the performance requirements (weighted MMF). Let us recall that MMF power control consists in maximizing the minimum SE in the network while providing identical SE to all the users. Considering the system model in Section 3.2.3, the power control optimization problem subject to per-AP power constraints, is formulated as follows

$$
\begin{array}{ll}
\underset{\left\{\eta_{m k} \geq 0\right\}}{\operatorname{maximize}} & \min _{k} \mathrm{SINR}_{\mathrm{d}, k} \\
\text { subject to } & \sum_{k=1}^{K} \eta_{m k} \gamma_{m k} \leq 1, \forall m .
\end{array}
$$

Clearly, this optimization is carried out in a centralized fashion at the CPU where the channel statistics from all the APs are available.

To preserve the scalability of the system, we propose power control approaches that are either heuristic and fully distributed (Paper B) or optimal and centralized (Paper C, Paper D, Paper E). The common ground is that these schemes only rely on large-scale fading quantities.

Figure 6 shows the impact of the power control on the downlink minimum SE per user. In these simulations, we consider $K=20$ single-antenna users, and $M=100$ single-antenna APs distributed over an area of $200 \times 200 \mathrm{~m}^{2}$. Pilots are assigned randomly and reused throughout the network as $\tau_{\mathrm{p}}=10<\mathrm{K}$. Finally, dynamic clustering is performed by using the largest-large-scale-fadingbased AP selection proposed in [116] (and also adopted in most of our works). As a consequence of this clustering, each AP serves a subset of the $K$ users. Let $\mathcal{K}_{m}$ denote the set of indices of the users served by AP $m$, then $\mathcal{K}_{m} \subseteq\{1, \ldots, K\}$. The right most curve is the cumulative distribution function (cdf) of the SE achieved by optimal MMF, while the left most curves are cdfs of the SEs achieved by using three fully distributed power control strategies:

1. Equal power allocation is obtained by setting the power control coefficients as follows

$$
\eta_{m k}= \begin{cases}\left(\left|\mathcal{K}_{m}\right| \gamma_{m k}\right)^{-1}, & \text { if } k \in \mathcal{K}_{m} \\ 0, & \text { otherwise }\end{cases}
$$

where $\left|\mathcal{K}_{m}\right|$ denotes the cardinality of the set $\mathcal{K}_{m}$. This power control scheme is not efficient as it does not take into account the users' channel conditions. Hence, the users with good channel conditions are penalized.

2. The "equal power coefficients" scheme [58] is obtained by setting

$$
\eta_{m k}= \begin{cases}\left(\sum_{k^{\prime} \in \mathcal{K}_{m}} \gamma_{m k^{\prime}}\right)^{-1}, & \text { if } k \in \mathcal{K}_{m} \\ 0, & \text { otherwise }\end{cases}
$$




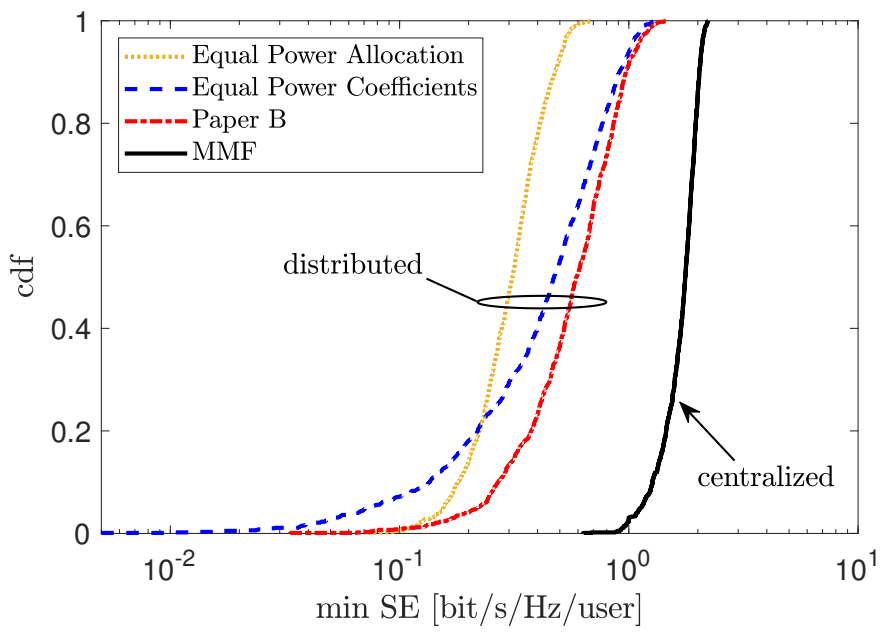

Figure 6: Cdf of the downlink min SE per user for different power control strategies.

which yields identical power control coefficients for a given $m$, that is $\eta_{m k}=$ $\eta_{m}, \forall k \in \mathcal{K}_{m}$ but not equal power allocation. In fact, by doing so, the effective power allocated to user $k$ is proportional to $\gamma_{m k}$ and normalized to satisfy the per-AP power constraint. Therefore, this choice allocates more power to the users with better channel conditions (in line with the waterfilling philosophy). Hence, the users with poor performance are further penalized.

3. The power control scheme proposed in Paper B is obtained by setting

$$
\eta_{m k}= \begin{cases}\left(\sqrt{\gamma_{m k}} \sum_{k^{\prime} \in \mathcal{K}_{m}} \sqrt{\gamma_{m k^{\prime}}}\right)^{-1}, & \text { if } k \in \mathcal{K}_{m} \\ 0, & \text { otherwise }\end{cases}
$$

where the allocated power is still proportional to the mean-square of the channel estimate but with a slower rate, that is proportional to $\sqrt{\gamma_{m k}}$. This choice offers a better fairness among the users, as shown in Figure 6, and performs particularly good if accompanied by a clustering scheme, which preliminary throws out the users with very poor channel conditions.

In [127] a downlink counterpart of the uplink fractional power allocation employed in current mobile standards was proposed for cell-free massive MIMO systems. This policy enables a trade-off between average performance and fairness by adjusting two parameters. The minimum SE achieved by this scheme may collocate between that achieved by the scheme in Paper B and the optimal MMF 
SE, upon the right choice of the parameters. However, the power policy in [127] requires statistical CSI exchange among the APs. Centralized deep learning-based power control strategies that get closer to the optimal MMF have been proposed in [128-130]. Methods for downlink power control are investigated in [131,132] aiming to reduce the complexity of existing solutions based on second-order optimization. Partial distributed downlink power control schemes based on inverse leakage and successive convex approximation are proposed in [133].

\section{Does the Channel Sufficiently Harden?}

In co-located massive MIMO, the distance from a user to each of the BS antennas is (approximately) the same. This results in scalar channels with identical large-scale fading properties but different small-scale fading coefficients. As the number of BS antennas grows, the fluctuations of the channel around its mean reduce and the effect of the small-scale fading vanishes by virtue of the law of the large numbers (the channel hardens). Conversely, in cell-free massive MIMO the antennas are geographically distributed, hence the channels towards a user have different largescale fading properties. As a consequence, the effective scalar channel seen at the user might not sufficiently harden. This intuition led us to investigate whether a pilot-based downlink training is beneficial in terms of spectral efficiency (Paper D). Again, when designing this scheme, we ensured not to jeopardize the scalability of the system. Hence, we devised a non-conventional downlink training whose resource requirements are proportional to the number of users instead of the antennas.

In this regard, we show in what extent the channel might harden in cell-free massive MIMO for the (best) case of independent Rayleigh fading. Let $\boldsymbol{g}_{k} \in \mathbb{C}^{M}$ be the vector collecting the scalar channels from $M$ single-antenna APs to user $k$. Based on the definition of channel hardening in (14), and according to [40], a necessary condition for channel hardening to hold is given by,

$$
\frac{\operatorname{Var}\left\{\left\|\boldsymbol{g}_{k}\right\|^{2}\right\}}{\left(\mathbb{E}\left\{\left\|\boldsymbol{g}_{k}\right\|^{2}\right\}\right)^{2}} \rightarrow 0, \quad \text { as } M \rightarrow \infty .
$$

Hence, a measure of the channel hardening degree $(\mathrm{ChD})$ at the $k$ th user can be defined as

$$
\mathrm{ChD}_{k}=1-\frac{\operatorname{Var}\left\{\sum_{m=1}^{M}\left|g_{m k}\right|^{2}\right\}}{\left(\mathbb{E}\left\{\sum_{m=1}^{M}\left|g_{m k}\right|^{2}\right\}\right)^{2}}=1-\frac{\sum_{m=1}^{M} \beta_{m k}^{2}}{\left(\sum_{m=1}^{M} \beta_{m k}\right)^{2}} .
$$

Hence, the larger the $\mathrm{ChD}_{k}$ is, the more the channel hardens for user $k$. 


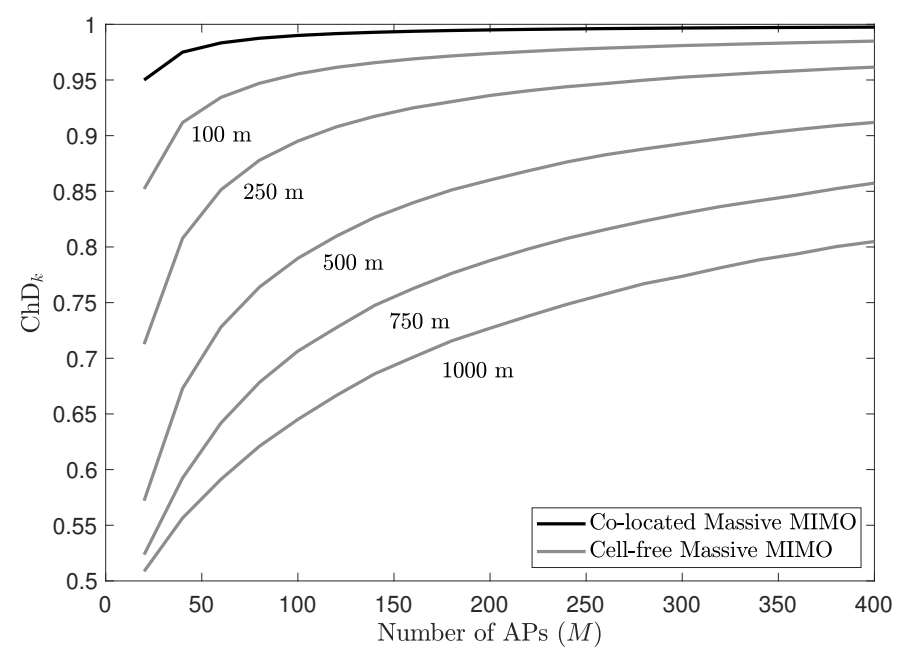

Figure 7: The average channel hardening degree versus different single-antenna AP densities. The values in the figure indicate the side lengths in meters of a square area. Here, the large-scale fading is modeled as in [58].

Figure 7 compares the channel hardening degree (averaged over many largescale fading realizations) at the $k$ th user for different single-antenna AP densities, assuming the three-slope path loss model in [58]. For co-located Massive MIMO, $\mathrm{ChD}_{k}=1-1 / M$, as a result of (15), and the channel hardening degree converges rapidly to 1 . Conversely, in cell-free Massive MIMO, the channel hardening degree converges slowly to 1 . Even increasing significantly the AP density does not help to achieve the same degree of channel hardening as for co-located Massive MIMO. Similarly, Figure 8 shows the channel hardening degree versus the number of APs, for different number of AP antennas. Increasing the number of antennas per AP, while keeping the total number of antennas fixed, boosts the channel hardening. Co-locating the antennas is beneficial from a channel hardening perspective but it comes at the expense of a decreased macro-diversity gain.

In the earlier example, the effective channel seen at user $k$ is $\left\|\boldsymbol{g}_{k}\right\|^{2}$ which entails MR precoding and perfect CSI. Channel hardening does depend on the precoding schemes adopted at the AP. Hence, a way to boost the channel hardening is to act on the precoding scheme by introducing a normalization term that equalizes the effective downlink channel (Paper E). The effect of the normalization term is to make the effective channel nearly deterministic thereby positively affecting the tightness of the hardening capacity bound and eliminating the need for downlink pilots in a simple way.

A modified conjugate beamforming scheme boosting the channel hardening at 


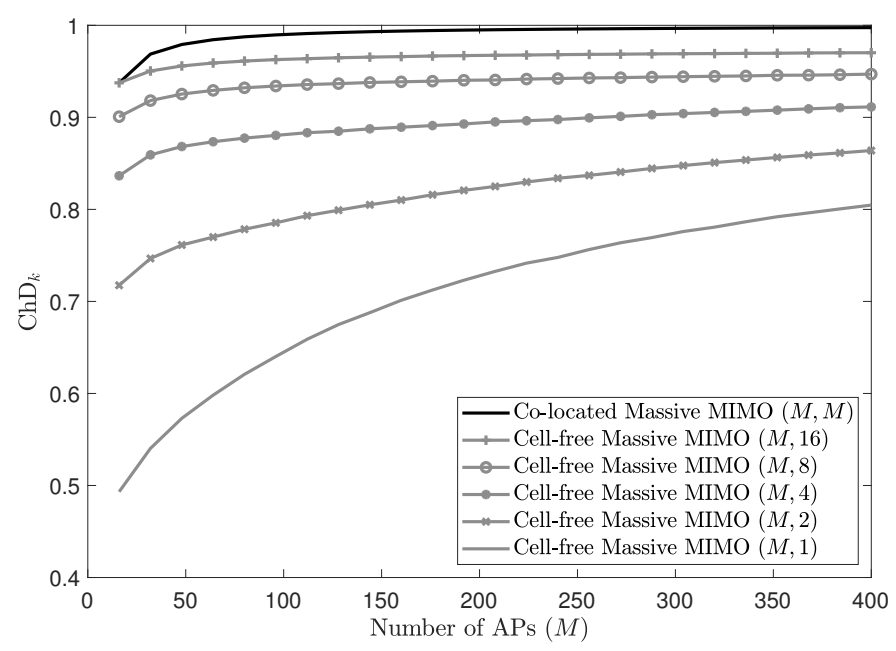

Figure 8: The average channel hardening degree versus the number of APs, for different numbers of antennas per AP. In the legend, the term $(X, Y)$ describes a system setup consisting of $X$ antennas in total, and $Y$ antennas per AP. For instance, co-located Massive MIMO is described by $(M, M)$, while cell-free Massive MIMO with single-antenna APs is described by $(M, 1)$. Here, the large-scale fading is modeled as in [58].

the user but requiring CSI exchange among the APs was proposed in [134]. Insights on channel hardening in cell-free massive MIMO are also provided in $[83,135,136]$.

\section{Scalability: A Framework for Pilot Assignment}

We emphasized many times in this introductory part of the thesis that AP clustering is crucial to maintain the system scalability. Selecting the right clusters to implement a user-centric transmission is a leitmotif in our works. In particular, dynamic clustering should take into account the underlying pre-determined clusters that are established by the physical links, while signal processing and power control should be able to smoothly adapt to the dynamic clustering (Paper B). A $K$-means clustering algorithm is proposed in [137] to minimize the pilot contamination within each cluster. [138] devises a clusterization based on the degree of channel hardening, while $[116,139]$ proposes AP selection methods based on the large-scale fading coefficients.

One aspect we did not include in the scalable framework of Paper B is the pilot assignment. This is presented next and it is based on the scheme proposed in [123].

Consider a cell-free network partitioned into disjoint (physical) clusters, each one mastered by a CPU. Dynamic cooperation clusters overlay the pre-determined clusters to implement user-centric transmission, as described in Paper B. In our 
vision, a scalable pilot assignment in such a scenario must be carried out locally at each cluster and should be optimized through local pilot re-allocations requiring a negotiation between neighboring CPUs. Given an initial local random pilot assignment, a CPU can compute, for each user $k$ served by one of its APs $m$, the metric

$$
P_{m k}=\frac{\sum_{k^{\prime} \in \mathcal{K}_{m}} \beta_{m k^{\prime}}\left|\boldsymbol{\phi}_{k}^{\mathrm{H}} \boldsymbol{\phi}_{k^{\prime}}\right|^{2}}{\beta_{m k}},
$$

which gives a measure of the pilot contamination at user $k$ from AP $m$ viewpoint. Based on this metric, the CPU detects the need for pilot re-assignment for more than one user (the larger $P_{m k}$ is the stronger this need is). Next, the CPU computes a list of the most preferable pilot sequences for each needy user (e.g., those pilots minimizing $P_{m k}$ ), and shares this list with the neighboring CPUs, requesting for pilot contamination measurements to evaluate the impact of the intended change on the users served by these neighboring CPUs. The neighboring CPUs can either reply with an ACK to permit the intended pilot re-assignment or with a NACK to deny. A reason to deny may be that the pilot re-assignment would cause additional pilot contamination in the neighboring clusters.

Figure 9 shows the gross ${ }^{1}$ downlink SE achieved by our scalable pilot allocation (proposed) with respect to the random pilot allocation and the orthogonal pilot allocation (benchmark). In these simulations we consider $K=60$ single-antenna users, and $M=300$ single-antenna APs grouped into 16 clusters and distributed over an area of $500 \times 500 \mathrm{~m}^{2}$. Pilots are reused throughout the network as $\tau_{\mathrm{p}}=20<$ $K$ (for the orthogonal pilot case $\tau_{\mathrm{p}}=60$ ). Dynamic clustering is performed by using a simple criterion: for each user the best 5 APs are identified, and the union of the clusters involved by these selected APs defines the serving cooperation cluster. The adopted power control strategy is the "equal power coefficients" described earlier. Finally, we assume that a user $k$ needs a pilot re-assignment for AP $m$ if $P_{m k} \geq 100$, i.e., the pilot-interfering users have a combined SNR that is $20 \mathrm{~dB}$ larger than the ideal SNR in absence of pilot contamination. As shown in Figure 9, the proposed scheme improves the SE of the random pilot allocation for all the users, and approaches the performance of the orthogonal pilot assignment. ${ }^{2}$ For instance, the $95 \%$-likely SE obtained with the proposed method is about $15 \%$ larger than that of the random pilot allocation scheme and roughly $10 \%$ smaller than that of the orthogonal pilot assignment.

Any sophisticated pilot assignment scheme requires some degree of centralized control. A greedy algorithm for pilot assignment maximizing the minimum rate in

\footnotetext{
${ }^{1}$ The gross downlink $\mathrm{SE}$ is $\mathrm{SE}_{\mathrm{d}, k}$ given in Section 3.2.3 and disregarding the pre-log factor $1-\tau_{\mathrm{p}} / \tau_{\mathrm{c}}$.

${ }^{2}$ Note that we are not compensating for the increased training overhead, thus the effective (net) spectral efficiency for the orthogonal pilot assignment scheme is actually much lower.
} 


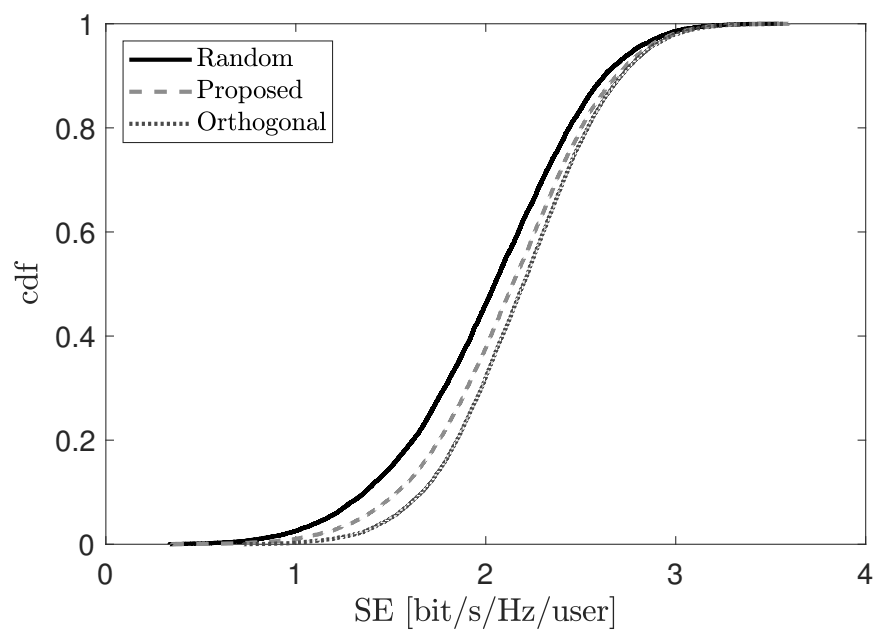

Figure 9: Cdf of the gross downlink SE per user for different pilot allocations.

the network is devised in [58]. While, the pilot reuse schemes in [140,141] reduce the pilot contamination by ensuring that the co-pilot users are enough spatially separated. A pilot allocation policy based on the tabu search, the Hungarian algorithm, and the graph coloring are presented in [142-144], respectively.

\section{Statistical CSI is Valuable}

The knowledge of the statistical CSI (i.e., the large-scale fading quantities) is crucial for manifold reasons. For instance, at the CPU, this knowledge can be exploited to optimize resource allocation tasks such as power control and pilot assignment, and to perform dynamic cooperation clustering. At the APs, this information constitutes the priors needed to perform Bayesian channel estimation methods such as MMSE. In addition, the APs can estimate an achievable SE for the transmission thereby enabling outage-free communications. Finally, at the user, the knowledge of the channel distribution allows "statistical" data detection, which is reliable if the effective fading channel is nearly deterministic.

In our works, we assume that the statistical CSI is available a priori wherever required. At the AP, the estimation of the statistical CSI is conventionally performed via orthogonal pilot resources to prevent correlation among the channel observations. In LoS scenarios, where the channels are highly correlated over time and frequency, estimation of the channel statistics can surprisingly be effective in presence of pilot contamination, provided that the pilots are appropriately phaserotated such that the contaminated channel observations becomes approximately 
de-correlated. This is the main finding of Paper F.

\section{Out of Scope}

In our works we make the following assumptions:

- infinite-capacity front/backhaul,

- perfect channel reciprocity and synchronization,

- single-antenna users,

- i.i.d. Rayleigh fading (but in Paper F we also consider LoS channels),

- no hardware impairments,

- analog-to-digital converters (ADCs) with high resolution,

and mainly look at the downlink of sub-6GHz systems. In this regard, Table 2 reports relevant related works studying some aspects of cell-free massive MIMO that are not addressed in this thesis. 


\begin{tabular}{|c|c|}
\hline Topics & Aspects and References \\
\hline \multirow[t]{6}{*}{ Uplink processing } & MMSE $[59,124,145,146]$ \\
\hline & $\mathrm{ZF}[147]$ \\
\hline & Power allocation and load balancing [148] \\
\hline & Max-min power control [149] \\
\hline & Pilot power control [150] \\
\hline & Sequential linear MMSE [120] \\
\hline \multirow[t]{4}{*}{ mmWave } & Limited fronthaul capacity [151] \\
\hline & Power control $[152]$ \\
\hline & Channel modeling [153] \\
\hline & Energy efficiency [154] \\
\hline Energy Efficiency & {$[116,132,155-157]$} \\
\hline Imperfect Fronthaul & {$[158-164]$} \\
\hline Low Resolution ADCs & {$[163,165-167]$} \\
\hline Hardware Impairments & {$[164,168-170]$} \\
\hline Non-orthogonal & {$[171-175]$} \\
\hline Multiple Access & \\
\hline Deep Learning & {$[176-180]$} \\
\hline \multirow[t]{2}{*}{ Channel Model } & Rician fading [181-183] \\
\hline & Spatially correlated Rayleigh fading [184-186] \\
\hline \multirow[t]{10}{*}{ Miscellaneous } & Full-duplex [187-189] \\
\hline & Channel non-reciprocity [190] \\
\hline & Asynchronous reception [191] \\
\hline & System information broadcast [192] \\
\hline & Over-the-air signaling $[193,194]$ \\
\hline & Multi-antenna users $[195,196]$ \\
\hline & Frequency division duplex [197] \\
\hline & Stochastic geometry [198] \\
\hline & Dynamic Resource allocation [199] \\
\hline & Wireless power transfer [200] \\
\hline
\end{tabular}

Table 2: Cell-free massive MIMO literature: aspects outside the scope of this thesis 


\section{Chapter 5}

\section{Conclusions and Future Directions}

\section{Final Considerations}

Cell-free massive MIMO is potentially a key enabler for beyond-5G technology, but soon can come to play an important role also for enhancing $5 \mathrm{G}$ evolution. It constitutes the perfect mix between network MIMO and massive MIMO while it takes over the heritage of CoMP. Over the last few years, cell-free massive MIMO has received an increasing attention from the academic research which mainly aimed to make this concept practical.

Now that many aspects have been theoretically addressed and the potential widely recognized, the industrial world is ready to pick up the baton and invest into this paradigm shift. So far, wireless networks have been conceived to be cell-centric, that is consisting of a base station serving all the users in its coverage area, referred to as cell. Only the lucky users standing close to the base station experience excellent quality of service.

Cell-free massive MIMO changes the perspective: all the users are equally important, each one in the center of its own tailored cell served by many access points. This is the principle behind the so-called user-centric architecture. The main technical challenge is enabling a smooth coordination and synchronization among the access points to implement such a user-centric service. The key for a successful implementation is to preserve the scalability of the system, that is the ability of the system to deal with an increasing number of users and access points.

Throughout this dissertation, we gave three main directions towards the scalable cell-free massive MIMO system: ( $i$ ) AP clustering, in order to confine data sharing and resource allocation within few useful access points; (ii) decentral- 
ization of channel estimation and precoding/combining, in order to offload the fronthaul network; and (iii) large-scale-fading-based power control. Power control is key to ensure high performance. Either it is performed in a distributed or centralized fashion, optimal and/or sub-optimal schemes must rely only on the large-scale fading quantities, that is the channel statistics.

\section{What Comes Next?}

Looking forward, the most relevant steps towards a cell-free user-centric world concern:

- Compliance with existing standards. Current core and access networks must be upgraded and optimized to better support the high levels of coordination needed to cell-free massive MIMO. C-RAN, with network slicing functionality, might be the first mature architecture supporting cell-free massive MIMO.

- Prototype development. As mentioned in the text, there are emerging lowcost and flexible solutions for cell-free networks aiming to demonstrate the promising theoretical performance. An interesting direction involves millimeter waves technology, not only for the purpose of providing high rates but possibly to replace wired links with a more flexible, high-capacity mmWave fronthaul.

From a communication-theoretic perspective, many aspects have been investigated among those envisioned in Paper A, such as enhanced distributed precoding/combining schemes, techniques for system information broadcasting, etc. Future research directions include:

- Downlink power control. There is still a significant performance gap from distributed power control schemes relying on local channel estimates and the centralized optimal max-min fairness power allocation (see Figure 6). Enhanced power control schemes are needed to fill this gap, while permitting a distributed implementation without CSI sharing.

- Synchronization. The synchronization among the APs is crucial to enable joint coherent transmission. The impact of imperfect synchronization on the performance has not been deeply studied yet.

- Sequential signal processing. To justify the substantial fronthauling expenses, the fronthaul network may embed the APs arranged in a compute-andforward architecture (this is the principle of the radio stripes [80]). Channel 
estimation, precoding/combining and power control schemes based on the sequential processing of the signal are of practical interest and deserve further studies.

- Non-coherent detection. In scenarios where the channel does not harden and the cost of a pilot-based downlink training is not sustainable, non-coherent detection or even blind channel estimation might be the most effective solution. This aspect has not been properly addressed yet.

- Channel modeling. The near-field effects and the channel non-stationarities should be included in the analysis of cell-free massive MIMO setups with extremely large aperture arrays.

Cell-free massive MIMO is a relatively young concept, not as well developed as co-located massive MIMO, and there is still much to explore about it. The cell-free era has only just begun! 


\section{Bibliography}

[1] "Mobile data traffic surpasses voice," Ericsson, 2010. [Online]. Available: https://www.ericsson.com/en/press-releases/2010/3/mobile-datatraffic-surpasses-voice

[2] E. Dahlman, S. Parkvall, and J. Sköld, 5G NR: The Next Generation Wireless Access Technology, 1st ed. Academic Press, 2018.

[3] J. G. Andrews, S. Buzzi, W. Choi, S. V. Hanly, A. Lozano, A. C. K. Soong, and J. C. Zhang, "What will 5G be?" IEEE fournal on Selected Areas in Communications, vol. 32, no. 6, pp. 1065-1082, Jun. 2014.

[4] “Ericsson Mobility Report June 2020," Ericsson, 2020. [Online]. Available: https://www.ericsson.com/en/mobility-report/reports/june-2020

[5] IMT Vision - Framework and overall objectives of the future development of IMT for 2020 and beyond, ITU-R Std. M.2083-0, 2015.

[6] H. Chen, R. Abbas, P. Cheng, M. Shirvanimoghaddam, W. Hardjawana, W. Bao, Y. Li, and B. Vucetic, "Ultra-reliable low latency cellular networks: Use cases, challenges and approaches," IEEE Communications Magazine, vol. 56, no. 12, pp. 119-125, Dec. 2018.

[7] H. Ji, S. Park, J. Yeo, Y. Kim, J. Lee, and B. Shim, "Ultra-reliable and low-latency communications in 5G downlink: Physical layer aspects," IEEE Wireless Communications, vol. 25, no. 3, pp. 124-130, Jun. 2018.

[8] P. Schulz, M. Matthe, H. Klessig, M. Simsek, G. Fettweis, J. Ansari, S. A. Ashraf, B. Almeroth, J. Voigt, I. Riedel, A. Puschmann, A. Mitschele-Thiel, M. Muller, T. Elste, and M. Windisch, "Latency critical IoT applications in 5G: Perspective on the design of radio interface and network architecture," IEEE Communications Magazine, vol. 55, no. 2, pp. 70-78, Feb. 2017.

[9] A. Willig, K. Matheus, and A. Wolisz, "Wireless technology in industrial networks," Proceedings of the IEEE, vol. 93, no. 6, pp. 1130-1151, Jun. 2005.

[10] M. Luvisotto, Z. Pang, and D. Dzung, "Ultra high performance wireless control for critical applications: Challenges and directions," IEEE Transactions on Industrial Informatics, vol. 13, no. 3, pp. 1448-1459, Jun. 2017.

[11] G. P. Fettweis, "The tactile Internet: Applications and challenges," IEEE Vehicular Technology Magazine, vol. 9, no. 1, pp. 64-70, Mar. 2014. 
[12] K. S. Kim, D. K. Kim, C. Chae, S. Choi, Y. Ko, J. Kim, Y. Lim, M. Yang, S. Kim, B. Lim, K. Lee, and K. L. Ryu, "Ultrareliable and low-latency communication techniques for tactile Internet services," Proceedings of the IEEE, vol. 107, no. 2, pp. 376-393, Feb. 2019.

[13] C. Bockelmann, N. Pratas, H. Nikopour, K. Au, T. Svensson, Č. Stefanović, P. Popovski, and A. Dekorsy, "Massive machine-type communications in 5G: physical and MAC-layer solutions," IEEE Communications Magazine, vol. 54, no. 9, pp. 59-65, Sep. 2016.

[14] C. Bockelmann, N. K. Pratas, G. Wunder, S. Saur, M. Navarro, D. Gregoratti, G. Vivier, E. De Carvalho, Y. Ji, Č. Stefanović, P. Popovski, Q. Wang, M. Schellmann, E. Kosmatos, P. Demestichas, M. Raceala-Motoc, P. Jung, S. Stanczak, and A. Dekorsy, "Towards massive connectivity support for scalable mMTC communications in 5G networks," IEEE Access, vol. 6, pp. 28 969-28 992, 2018.

[15] L. Atzori, A. Iera, and G. Morabito, "The Internet of Things: A survey," Computer Networks, vol. 54, no. 15, pp. 2787 - 2805, Oct. 2010.

[16] X. Foukas, G. Patounas, A. Elmokashfi, and M. K. Marina, "Network slicing in 5G: Survey and challenges," IEEE Communications Magazine, vol. 55, no. 5, pp. 94-100, May 2017.

[17] H. Zhang, N. Liu, X. Chu, K. Long, A. Aghvami, and V. C. M. Leung, "Network slicing based 5G and future mobile networks: Mobility, resource management, and challenges," IEEE Communications Magazine, vol. 55, no. 8, pp. 138-145, Aug. 2017.

[18] I. Afolabi, T. Taleb, K. Samdanis, A. Ksentini, and H. Flinck, "Network slicing and softwarization: A survey on principles, enabling technologies, and solutions," IEEE Communications Surveys Tutorials, vol. 20, no. 3, pp. 24292453, 2018.

[19] J. Ordonez-Lucena, P. Ameigeiras, D. Lopez, J. J. Ramos-Munoz, J. Lorca, and J. Folgueira, "Network slicing for $5 \mathrm{G}$ with SDN/NFV: Concepts, architectures, and challenges," IEEE Communications Magazine, vol. 55, no. 5, pp. 80-87, May 2017.

[20] E. Björnson, J. Hoydis, and L. Sanguinetti, "Massive MIMO networks: Spectral, energy, and hardware efficiency," Foundations and Trends® in Signal Processing, vol. 11, no. 3-4, pp. 154-655, 2017. 
[21] V. Chandrasekhar, J. G. Andrews, and A. Gatherer, "Femtocell networks: A survey," IEEE Communications Magazine, vol. 46, no. 9, pp. 59-67, Sep. 2008.

[22] M. Dohler, R. W. Heath, A. Lozano, C. B. Papadias, and R. A. Valenzuela, "Is the PHY layer dead?" IEEE Communications Magazine, vol. 49, no. 4, pp. 159-165, Apr. 2011.

[23] D. Lopez-Perez, I. Guvenc, G. de la Roche, M. Kountouris, T. Quek, and J. Zhang, "Enhanced intercell interference coordination challenges in heterogeneous networks," IEEE Wireless Communications, vol. 18, no. 3, pp. 22-30, Jun. 2011.

[24] J. G. Andrews, X. Zhang, G. D. Durgin, and A. K. Gupta, "Are we approaching the fundamental limits of wireless network densification?" IEEE Communications Magazine, vol. 54, no. 10, pp. 184-190, Oct. 2016.

[25] E. Telatar, "Capacity of multi-antenna Gaussian channels," European transactions on telecommunications, vol. 10, no. 6, pp. 585-595, 1999.

[26] D. Tse and P. Viswanath, Fundamentals of wireless communication. Cambridge University Press, 2005.

[27] R. W. Heath Jr. and A. Lozano, Foundations of MIMO communication. Cambridge University Press, 2018.

[28] E. F. W. Alexanderson, "Transatlantic radio communication," Proceedings of the American Institute of Electrical Engineers, vol. 38, no. 10, pp. 1077-1093, Oct. 1919.

[29] S. C. Swales, M. A. Beach, D. J. Edwards, and J. P. McGeehan, "The performance enhancement of multibeam adaptive base-station antennas for cellular land mobile radio systems," IEEE Transactions on Vehicular Technology, vol. 39, no. 1, pp. 56-67, Feb. 1990.

[30] D. G. Brennan, "Linear diversity combining techniques," Proceedings of the IRE, vol. 47, no. 6, pp. 1075-1102, 1959.

[31] E. G. Larsson, O. Edfors, F. Tufvesson, and T. L. Marzetta, "Massive MIMO for next generation wireless systems," IEEE Communications Magazine, vol. 52, no. 2, pp. 186-195, Feb. 2014.

[32] H. Q. Ngo, "Massive MIMO: Fundamentals and system designs," PhD disseratation, Linköping University, 2015. 
[33] T. L. Marzetta, E. G. Larsson, H. Yang, and H. Q. Ngo, Fundamentals of massive MIMO. Cambridge University Press, 2016.

[34] F. Rusek, D. Persson, B. K. Lau, E. G. Larsson, T. L. Marzetta, O. Edfors, and F. Tufvesson, "Scaling up MIMO: Opportunities and challenges with very large arrays," IEEE Signal Processing Magazine, vol. 30, no. 1, pp. 40-60, Jan. 2013.

[35] T. L. Marzetta, "Noncooperative cellular wireless with unlimited numbers of base station antennas," IEEE Transactions on Wireless Communications, vol. 9, no. 11, pp. 3590-3600, Nov. 2010.

[36] E. Björnson, E. G. Larsson, and T. L. Marzetta, "Massive MIMO: Ten myths and one critical question," IEEE Communications Magazine, vol. 54, no. 2, pp. 114-123, Feb. 2016.

[37] H. Q. Ngo, E. G. Larsson, and T. L. Marzetta, "Aspects of favorable propagation in massive MIMO," in 2014 22nd European Signal Processing Conference (EUSIPCO), Sep. 2014, pp. 76-80.

[38] À. O. Martínez, E. De Carvalho, and J. Ø. Nielsen, "Massive MIMO properties based on measured channels: Channel hardening, user decorrelation and channel sparsity," in 2016 50th Asilomar Conference on Signals, Systems and Computers, Nov. 2016, pp. 1804-1808.

[39] S. Gunnarsson, J. Flordelis, L. Van Der Perre, and F. Tufvesson, "Channel hardening in massive MIMO: Model parameters and experimental assessment," IEEE Open fournal of the Communications Society, vol. 1, pp. 501-512, 2020.

[40] H. Q. Ngo and E. G. Larsson, "No downlink pilots are needed in TDD massive MIMO," IEEE Transactions on Wireless Communications, vol. 16, no. 5, pp. 2921-2935, May 2017.

[41] H. Q. Ngo, E. G. Larsson, and T. L. Marzetta, "Energy and spectral efficiency of very large multiuser MIMO systems," IEEE Transactions on Communications, vol. 61, no. 4, pp. 1436-1449, Apr. 2013.

[42] E. Björnson, E. G. Larsson, and M. Debbah, "Massive MIMO for maximal spectral efficiency: How many users and pilots should be allocated?" IEEE Transactions on Wireless Communications, vol. 15, no. 2, pp. 1293-1308, Feb. 2016. 
[43] A. Zappone, L. Sanguinetti, G. Bacci, E. Jorswieck, and M. Debbah, "Energyefficient power control: A look at 5G wireless technologies," IEEE Transactions on Signal Processing, vol. 64, no. 7, pp. 1668-1683, Apr. 2016.

[44] H. V. Cheng, E. Björnson, and E. G. Larsson, "Optimal pilot and payload power control in single-cell massive MIMO systems," IEEE Transactions on Signal Processing, vol. 65, no. 9, pp. 2363-2378, May 2017.

[45] X. Li, E. Björnson, E. G. Larsson, S. Zhou, and J. Wang, "Massive MIMO with multi-cell MMSE processing: Exploiting all pilots for interference suppression," EURASIP fournal on Wireless Communications and Networking, vol. 2017, no. 1, p. 117, Jun. 2017.

[46] E. Nayebi, A. Ashikhmin, T. L. Marzetta, H. Yang, and B. D. Rao, "Precoding and power optimization in cell-free massive MIMO systems," IEEE Transactions on Wireless Communications, vol. 16, no. 7, pp. 4445-4459, Jul. 2017.

[47] H. Yang and T. L. Marzetta, "Massive MIMO with max-min power control in line-of-sight propagation environment," IEEE Transactions on Communications, vol. 65, no. 11, pp. 4685-4693, Nov. 2017.

[48] T. Van Chien, E. Björnson, and E. G. Larsson, "Joint pilot design and uplink power allocation in multi-cell massive MIMO systems," IEEE Transactions on Wireless Communications, vol. 17, no. 3, pp. 2000-2015, Mar. 2018.

[49] A. Ghazanfari, H. V. Cheng, E. Björnson, and E. G. Larsson, "Enhanced fairness and scalability of power control schemes in multi-cell massive MIMO," IEEE Transactions on Communications, vol. 68, no. 5, pp. 2878-2890, May 2020.

[50] X. Zhu, Z. Wang, L. Dai, and C. Qian, "Smart pilot assignment for massive MIMO,” IEEE Communications Letters, vol. 19, no. 9, pp. 1644-1647, Sep. 2015.

[51] X. Zhu, L. Dai, and Z. Wang, "Graph coloring based pilot allocation to mitigate pilot contamination for multi-cell massive MIMO systems," IEEE Communications Letters, vol. 19, no. 10, pp. 1842-1845, Oct. 2015.

[52] S. Jin, M. Li, Y. Huang, Y. Du, and X. Gao, "Pilot scheduling schemes for multi-cell massive multiple-input-multiple-output transmission," IET Communications, vol. 9, no. 5, pp. 689-700, 2015.

[53] H. Ahmadi, A. Farhang, N. Marchetti, and A. MacKenzie, "A game theoretic approach for pilot contamination avoidance in massive MIMO," IEEE Wireless Communications Letters, vol. 5, no. 1, pp. 12-15, Feb. 2016. 
[54] F. Boccardi, R. W. Heath, A. Lozano, T. L. Marzetta, and P. Popovski, "Five disruptive technology directions for 5G," IEEE Communications Magazine, vol. 52, no. 2, pp. 74-80, Feb. 2014.

[55] "Ericsson first to deliver 5G NR radio," Ericsson, 2016. [Online]. Available: https://www.ericsson.com/en/press-releases/2016/8/ericsson-firstto-deliver-5g-nr-radio

[56] H. Q. Ngo, A. Ashikhmin, H. Yang, E. G. Larsson, and T. L. Marzetta, "Cellfree massive MIMO: Uniformly great service for everyone," in 2015 IEEE 16th International Workshop on Signal Processing Advances in Wireless Communications (SPAWC), Jun. 2015, pp. 201-205.

[57] E. Nayebi, A. Ashikhmin, T. L. Marzetta, and H. Yang, "Cell-free massive MIMO systems," in 2015 49th Asilomar Conference on Signals, Systems and Computers, Nov. 2015, pp. 695-699.

[58] H. Q. Ngo, A. Ashikhmin, H. Yang, E. G. Larsson, and T. L. Marzetta, "Cell-free massive MIMO versus small cells," IEEE Transactions on Wireless Communications, vol. 16, no. 3, pp. 1834-1850, Mar. 2017.

[59] E. Björnson and L. Sanguinetti, "Making cell-free massive MIMO competitive with MMSE processing and centralized implementation," IEEE Transactions on Wireless Communications, vol. 19, no. 1, pp. 77-90, Jan. 2020.

[60] S. Shamai and B. M. Zaidel, "Enhancing the cellular downlink capacity via co-processing at the transmitting end," in IEEE VTS 53rd Vehicular Technology Conference, Spring 2001., vol. 3, May 2001, pp. 1745-1749.

[61] S. Zhou, M. Zhao, X. Xu, J. Wang, and Y. Yao, "Distributed wireless communication system: A new architecture for future public wireless access," IEEE Communications Magazine, vol. 41, no. 3, pp. 108-113, Mar. 2003.

[62] G. Foschini, "The value of coherent base station coordination," in Conference on Information Sciences and Systems (CISS), Mar. 2005.

[63] M. Karakayali, G. Foschini, and R. Valenzuela, "Network coordination for spectrally efficient communications in cellular systems," IEEE Wireless Communications, vol. 13, no. 4, pp. 56-61, Aug. 2006.

[64] S. Venkatesan, A. Lozano, and R. Valenzuela, "Network MIMO: Overcoming intercell interference in indoor wireless systems," in 2007 41st Asilomar Conference on Signals, Systems and Computers, Nov. 2007, pp. 83-87. 
[65] G. Caire, S. Ramprashad, and H. Papadopoulos, "Rethinking network MIMO: Cost of CSIT, performance analysis, and architecture comparisons," in 2010 Information Theory and Applications Workshop (ITA), Jan. 2010, pp. 1-10.

[66] W. Choi and J. G. Andrews, "Downlink performance and capacity of distributed antenna systems in a multicell environment," IEEE Transactions on Wireless Communications, vol. 6, no. 1, pp. 69-73, Jan. 2007.

[67] A. Tölli, M. Codreanu, and M. Juntti, "Cooperative MIMO-OFDM cellular system with soft handover between distributed base station antennas," IEEE Transactions on Wireless Communications, vol. 7, no. 4, pp. 1428-1440, 2008.

[68] D. Gesbert, S. Hanly, H. Huang, S. Shamai, O. Simeone, and W. Yu, "Multi-cell MIMO cooperative networks: A new look at interference," IEEE fournal on Selected Areas in Communications, vol. 28, no. 9, pp. 1380-1408, Dec. 2010.

[69] W. Feng, Y. Wang, N. Ge, J. Lu, and J. Zhang, "Virtual MIMO in multi-cell distributed antenna systems: Coordinated transmissions with large-scale CSIT," IEEE Journal on Selected Areas in Communications, vol. 31, no. 10, pp. 2067-2081, Oct. 2013.

[70] R. Irmer, H. Droste, P. Marsch, M. Grieger, G. Fettweis, S. Brueck, H.-P. Mayer, L. Thiele, and V. Jungnickel, "Coordinated multipoint: Concepts, performance, and field trial results," IEEE Communications Magazine, vol. 49, no. 2, pp. 102-111, Feb. 2011.

[71] M. Boldi, A. Tölli, M. Olsson, E. Hardouin, T. Svensson, F. Boccardi, L. Thiele, and V. Jungnickel, "Coordinated multipoint (CoMP) systems," in Mobile and Wireless Communications for IMT-Advanced and Beyond, A. Osseiran, J. Monserrat, and W. Mohr, Eds. Wiley, 2011, pp. 121-155.

[72] P. Marsch, S. Brück, A. Garavaglia, M. Schulist, R. Weber, and A. Dekorsy, "Clustering," in Coordinated multi-point in mobile communications: From theory to practice, P. Marsch and G. Fettweis, Eds. Cambridge University Press, 2011, pp. 139-159.

[73] A. Lozano, R. W. Heath, and J. G. Andrews, "Fundamental limits of cooperation," IEEE Journal on Selected Areas in Communications, vol. 59, no. 9, pp. 5213-5226, Sep. 2013.

[74] R. Fantini, W. Zirwas, L. Thiele, D. Aziz, and P. Baracca, "Coordinated multi-point transmission in 5G," in 5G Mobile and Wireless Communications Technology, A. Osseiran, J. Monserrat, and P. Marsch, Eds. Cambridge University Press, 2016, p. 248-276. 
[75] J. Zhang, S. Chen, Y. Lin, J. Zheng, B. Ai, and L. Hanzo, "Cell-free massive MIMO: A new next-generation paradigm," IEEE Access, vol. 7, pp. $99878-$ 99 888, Jul. 2019.

[76] E. Björnson, L. Sanguinetti, H. Wymeersch, J. Hoydis, and T. L. Marzetta, "Massive MIMO is a reality-What is next?: Five promising research directions for antenna arrays," Digital Signal Processing, vol. 94, pp. 3-20, Nov. 2019.

[77] J. Zhang, E. Björnson, M. Matthaiou, D. W. K. Ng, H. Yang, and D. J. Love, "Prospective multiple antenna technologies for beyond 5G," IEEE Journal on Selected Areas in Communications, pp. 1-1, 2020, early access.

[78] N. Rajatheva, I. Atzeni, E. Björnson, A. Bourdoux, S. Buzzi, J.-B. Dore, S. Erkucuk, M. Fuentes, K. Guan, Y. Hu et al., "White paper on broadband connectivity in 6G," arXiv preprint arXiv:2004.14247, 2020.

[79] M. Matthaiou, O. Yurduseven, H. Q. Ngo, D. Morales-Jimenez, S. L. Cotton, and V. F. Fusco, "The road to 6G: Ten physical layer challenges for communications engineers," arXiv preprint arXiv:2004.07130, 2020.

[80] P. Frenger, J. Hederen, M. Hessler, and G. Interdonato, "Antenna arrangement for distributed massive MIMO,” US Patent App. 16/435,054, Nov. 2019. [Online]. Available: https://patents.google.com/patent/US20190363763A1

[81] P. Frenger, G. Interdonato, and E. G. Larsson, "Methods and apparatuses for cell-free massive MIMO communication," WO Patent App. 2020076203A1, Apr. 2020. [Online]. Available: https://patents.google.com/patent/WO2020076203A1

[82] E. Björnson, P. Frenger, G. Interdonato, M. Karlsson, and E. G. Larsson, "Disturbance mitigation," US Patent App. 20200119772A1, Apr. 2020. [Online]. Available: https://patents.google.com/patent/US20200119772A1

[83] Z. Chen and E. Björnson, "Channel hardening and favorable propagation in cell-free massive MIMO with stochastic geometry," IEEE Transactions on Communications, vol. 66, no. 11, pp. 5205-5219, Nov. 2018.

[84] C. E. Shannon, "A mathematical theory of communication," Bell System Technical fournal, vol. 27, pp. 379-423, 623-656, 1948.

[85] T. M. Cover and J. A. Thomas, Elements of information theory. Wiley, 1991. 
[86] V. Tarokh, H. Jafarkhani, and A. Calderbank, "Space-time block codes from orthogonal designs," IEEE Transactions on Information Theory, vol. 45, no. 5, pp. 1456-1467, 1999.

[87] E. Larsson and P. Stoica, Space-time block coding for wireless communications. Cambridge University Press, 2003.

[88] S. Alamouti, "A simple transmit diversity technique for wireless communications," IEEE Journal on Selected Areas in Communications, vol. 16, no. 8, pp. 1451-1458, Oct. 1998.

[89] G. Raleigh and J. Cioffi, "Spatio-temporal coding for wireless communication," IEEE Transactions on Communications, vol. 46, no. 3, pp. 357-366, Mar. 1998.

[90] G. J. Foschini and M. J. Gans, "On limits of wireless communications in a fading environment when using multiple antennas," Wireless personal communications, vol. 6, no. 3, pp. 311-335, 1998.

[91] E. Dahlman, S. Parkvall, and J. Sköld, 4G: LTE/LTE-Advanced for Mobile Broadband, 2nd ed. Academic Press, 2013.

[92] S. Verdú, Multiuser Detection. Cambridge University Press, 1998.

[93] M. Costa, "Writing on dirty paper," IEEE Transactions on Information Theory, vol. 29, no. 3, pp. 439-441, May 1983.

[94] A. Goldsmith, S. A. Jafar, N. Jindal, and S. Vishwanath, "Capacity limits of MIMO channels," IEEE Journal on Selected Areas in Communications, vol. 21, no. 5, pp. 684-702, Jun. 2003.

[95] G. Caire and S. Shamai, "On the achievable throughput of a multiantenna Gaussian broadcast channel," IEEE Transactions on Information Theory, vol. 49, no. 7, pp. 1691-1706, Jul. 2003.

[96] P. Viswanath and D. N. C. Tse, "Sum capacity of the vector Gaussian broadcast channel and uplink-downlink duality," IEEE Transactions on Information Theory, vol. 49, no. 8, pp. 1912-1921, Aug. 2003.

[97] X. Wu, N. C. Beaulieu, and D. Liu, "On favorable propagation in massive MIMO systems and different antenna configurations," IEEE Access, vol. 5, pp. 5578-5593, 2017.

[98] B. M. Hochwald, T. L. Marzetta, and V. Tarokh, "Multiple-antenna channel hardening and its implications for rate feedback and scheduling," IEEE Transactions on Information Theory, vol. 50, no. 9, pp. 1893-1909, Sep. 2004. 
[99] X. Gao, O. Edfors, F. Rusek, and F. Tufvesson, "Massive MIMO performance evaluation based on measured propagation data," IEEE Transactions on Wireless Communications, vol. 14, no. 7, pp. 3899-3911, Jul. 2015.

[100] J. Vieira, F. Rusek, O. Edfors, S. Malkowsky, L. Liu, and F. Tufvesson, "Reciprocity calibration for massive MIMO: Proposal, modeling, and validation," IEEE Transactions on Wireless Communications, vol. 16, no. 5, pp. 3042-3056, May 2017.

[101] H. Zhang and H. Dai, "Cochannel interference mitigation and cooperative processing in downlink multicell multiuser MIMO networks," EURASIP fournal on Wireless Communications and Networking, vol. 2004, no. 2, p. 202654, Dec. 2004.

[102] A. Papadogiannis, D. Gesbert, and E. Hardouin, "A dynamic clustering approach in wireless networks with multi-cell cooperative processing," in 2008 IEEE International Conference on Communications, May 2008, pp. 4033-4037.

[103] E. Björnson, R. Zakhour, D. Gesbert, and B. Ottersten, "Cooperative multicell precoding: Rate region characterization and distributed strategies with instantaneous and statistical CSI,' IEEE Transactions on Signal Processing, vol. 58, no. 8, pp. 4298-4310, Aug. 2010.

[104] I. Garcia, N. Kusashima, K. Sakaguchi, and K. Araki, "Dynamic cooperation set clustering on base station cooperation cellular networks," in 21st Annual IEEE International Symposium on Personal, Indoor and Mobile Radio Communications (PIMRC), Sep. 2010, pp. 2127-2132.

[105] E. Björnson and E. Jorswieck, Optimal resource allocation in coordinated multi-cell systems, ser. Foundations and Trends in Communications and Information Theory. Now Publishers Inc, 2013, vol. 9, no. 2-3.

[106] "Coordinated Multi-Point Operation for LTE Physical Layer Aspects," 3GPP, Dec. 2011, (Release 11) Version 11.1.0, 3GPP TR 36.819.

[107] V. Jungnickel, K. Manolakis, W. Zirwas, B. Panzner, V. Braun, M. Lossow, M. Sternad, R. Apelfröjd, and T. Svensson, "The role of small cells, coordinated multipoint, and massive MIMO in 5G," IEEE Communications Magazine, vol. 52, no. 5, pp. 44-51, May 2014.

[108] "C-RAN white paper: The road towards green RAN," China Mobile Research Institute, 2014. [Online]. Available: http://labs.chinamobile.com/cran 
[109] J. Yuan, S. Jin, W. Xu, W. Tan, M. Matthaiou, and K. Wong, "User-centric networking for dense C-RANs: High-SNR capacity analysis and antenna selection," IEEE Transactions on Communications, vol. 65, no. 11, pp. 50675080, Nov. 2017.

[110] C. Pan, M. Elkashlan, J. Wang, J. Yuan, and L. Hanzo, "User-centric C-RAN architecture for ultra-dense 5G networks: Challenges and methodologies," IEEE Communications Magazine, vol. 56, no. 6, pp. 14-20, Jun. 2018.

[111] À. O. Martínez, E. De Carvalho, and J. Ø. Nielsen, "Towards very large aperture massive MIMO: A measurement based study," in 2014 IEEE Globecom Workshops (GC Wkshps), Dec. 2014, pp. 281-286.

[112] A. Amiri, M. Angjelichinoski, E. de Carvalho, and R. W. Heath, "Extremely large aperture massive MIMO: Low complexity receiver architectures," in 2018 IEEE Globecom Workshops (GC Wkshps), Dec. 2018, pp. 1-6.

[113] E. D. Carvalho, A. Ali, A. Amiri, M. Angjelichinoski, and R. W. Heath, "Nonstationarities in extra-large-scale massive MIMO," IEEE Wireless Communications, vol. 27, no. 4, pp. 74-80, 2020.

[114] S. Payami and F. Tufvesson, "Channel measurements and analysis for very large array systems at $2.6 \mathrm{GHz}$," in 2012 6th European Conference on Antennas and Propagation (EUCAP), Mar. 2012, pp. 433-437.

[115] H. Wang, A. Kosasih, C. Wen, S. Jin, and W. Hardjawana, "Expectation propagation detector for extra-large scale massive MIMO," IEEE Transactions on Wireless Communications, vol. 19, no. 3, pp. 2036-2051, Mar. 2020.

[116] H. Q. Ngo, L. N. Tran, T. Q. Duong, M. Matthaiou, and E. G. Larsson, "On the total energy efficiency of cell-free massive MIMO," IEEE Transactions on Green Communications and Networking, vol. 2, no. 1, pp. 25-39, Mar. 2018.

[117] S. Buzzi, C. D’Andrea, A. Zappone, and C. D'Elia, “User-centric 5G cellular networks: Resource allocation and comparison with the cell-free massive MIMO approach," IEEE Transactions on Wireless Communications, vol. 19, no. 2, pp. 1250-1264, Feb. 2020.

[118] A. Forenza, S. Perlman, F. Saibi, M. Di Dio, R. van der Laan, and G. Caire, "Achieving large multiplexing gain in distributed antenna systems via cooperation with pCell technology," in 2015 49th Asilomar Conference on Signals, Systems and Computers, Nov. 2015, pp. 286-293. 
[119] S. Perlman and A. Forenza, "An introduction to pCell," Artemis Networks LLC, White paper, 2015. [Online]. Available: http://www.rearden.com/artemis/An-Introduction-to-pCell-WhitePaper-150224.pdf

[120] Z. H. Shaik, E. Björnson, and E. G. Larsson, "Cell-free massive MIMO with Radio Stripes and sequential uplink processing," in 2020 IEEE International Conference on Communications Workshops (ICC Workshops), Jun. 2020, pp. $1-6$.

[121] M. Medard, "The effect upon channel capacity in wireless communications of perfect and imperfect knowledge of the channel," IEEE Transactions on Information Theory, vol. 46, no. 3, pp. 933-946, May 2000.

[122] G. Borg, Z. Javaid, and A. Khandaker, "The physical and engineering requirements of scalable, decentralised, distributed, large-scale MIMO," in 2018 Third International Conference on Informatics and Computing (ICIC), Oct. 2018, pp. 1-6.

[123] E. Björnson and L. Sanguinetti, "Scalable cell-free massive MIMO systems," IEEE Transactions on Communications, vol. 68, no. 7, pp. 4247-4261, Jul. 2020.

[124] S. Chen, J. Zhang, E. Björnson, J. Zhang, and B. Ai, "Structured massive access for scalable cell-free massive MIMO systems," IEEE fournal on Selected Areas in Communications, pp. 1-1, 2020, early access.

[125] F. Riera-Palou and G. Femenias, "Decentralization issues in cell-free massive MIMO networks with zero-forcing precoding," in 2019 57th Annual Allerton Conference on Communication, Control, and Computing (Allerton), Sep. 2019, pp. 521-527.

[126] O. Y. Bursalioglu, G. Caire, R. K. Mungara, H. C. Papadopoulos, and C. Wang, "Fog massive MIMO: A user-centric seamless hot-spot architecture," IEEE Transactions on Wireless Communications, vol. 18, no. 1, pp. 559-574, Jan. 2019.

[127] R. Nikbakht, R. Mosayebi, and A. Lozano, "Uplink fractional power control and downlink power allocation for cell-free networks," IEEE Wireless Communications Letters, vol. 9, no. 6, pp. 774-777, Jun. 2020.

[128] S. Chakraborty, E. Björnson, and L. Sanguinetti, "Centralized and distributed power allocation for max-min fairness in cell-free massive MIMO," in 2019 53rd Asilomar Conference on Signals, Systems, and Computers, Nov. 2019, pp. 576-580. 
[129] R. Nikbakht, A. Jonsson, and A. Lozano, "Unsupervised-learning power control for cell-free wireless systems," in 2019 IEEE 30th Annual International Symposium on Personal, Indoor and Mobile Radio Communications (PIMRC), Sep. 2019, pp. 1-5.

[130] Y. Zhao, I. G. Niemegeers, and S. H. De Groot, "Power allocation in cell-free massive MIMO: A deep learning method," IEEE Access, vol. 8, pp. 87 185$87200,2020$.

[131] L. Tran and H. Q. Ngo, "First-order methods for energy-efficient power control in cell-free massive MIMO: Invited paper," in 2019 53rd Asilomar Conference on Signals, Systems, and Computers, Nov. 2019, pp. 848-852.

[132] T. Van Chien, E. Björnson, and E. G. Larsson, "Joint power allocation and load balancing optimization for energy-efficient cell-free massive MIMO networks," IEEE Transactions on Wireless Communications, pp. 1-1, 2020, early access.

[133] J. Francis, P. Baracca, S. Wesemann, and G. Fettweis, "Downlink power control in cell-free massive MIMO with partially distributed access points," in 2019 IEEE 90th Vehicular Technology Conference (VTC2019-Fall), Sep. 2019, pp. $1-7$.

[134] M. Attarifar, A. Abbasfar, and A. Lozano, "Modified conjugate beamforming for cell-free massive MIMO," IEEE Wireless Communications Letters, vol. 8, no. 2, pp. 616-619, Apr. 2019.

[135] A. A. Polegre, F. Riera-Palou, G. Femenias, and A. G. Armada, "New insights on channel hardening in cell-free massive MIMO networks," in 2020 IEEE International Conference on Communications Workshops (ICC Workshops), Jun. 2020, pp. 1-7.

[136] - -, "Channel hardening in cell-free and user-centric massive MIMO networks with spatially correlated Ricean fading," IEEE Access, pp. 1-1, 2020, early access.

[137] F. Riera-Palou, G. Femenias, A. G. Armada, and A. Pérez-Neira, "Clustered cell-free massive MIMO," in 2018 IEEE Globecom Workshops (GC Wkshps), Dec. 2018, pp. 1-6.

[138] A. A. Polegre and A. G. Armada, "User-centric massive MIMO systems with hardening-based clusterization," in 2020 24th International ITG Workshop on Smart Antennas (WSA), Feb. 2020, pp. 1-5. 
[139] H. T. Dao and S. Kim, "Effective channel gain-based access point selection in cell-free massive MIMO systems," IEEE Access, vol. 8, pp. 108 127-108 132, 2020.

[140] M. Attarifar, A. Abbasfar, and A. Lozano, "Random vs structured pilot assignment in cell-free massive MIMO wireless networks," in 2018 IEEE International Conference on Communications Workshops (ICC Workshops), May 2018, pp. 1-6.

[141] R. Sabbagh, C. Pan, and J. Wang, "Pilot allocation and sum-rate analysis in cell-free massive MIMO systems," in 2018 IEEE International Conference on Communications (ICC), May 2018, pp. 1-6.

[142] H. Liu, J. Zhang, X. Zhang, A. Kurniawan, T. Juhana, and B. Ai, "Tabusearch-based pilot assignment for cell-free massive MIMO systems," IEEE Transactions on Vehicular Technology, vol. 69, no. 2, pp. 2286-2290, Feb. 2020.

[143] S. Buzzi, C. D’Andrea, M. Fresia, Y.-P. Zhang, and S. Feng, "Pilot assignment in cell-free massive MIMO based on the Hungarian algorithm," arXiv preprint arXiv:2004.06940, 2020.

[144] H. Liu, J. Zhang, S. Jin, and B. Ai, "Graph coloring based pilot assignment for cell-free massive MIMO systems," IEEE Transactions on Vehicular Technology, pp. 1-1, 2020, early access.

[145] E. Nayebi, A. Ashikhmin, T. L. Marzetta, and B. D. Rao, "Performance of cell-free massive MIMO systems with MMSE and LSFD receivers," in 2016 50th Asilomar Conference on Signals, Systems and Computers, Nov. 2016, pp. 203-207.

[146] M. Attarifar, A. Abbasfar, and A. Lozano, "Subset MMSE receivers for cellfree networks," IEEE Transactions on Wireless Communications, vol. 19, no. 6, pp. 4183-4194, Jun. 2020.

[147] P. Liu, K. Luo, D. Chen, and T. Jiang, "Spectral efficiency analysis of cell-free massive MIMO systems with zero-forcing detector," IEEE Transactions on Wireless Communications, vol. 19, no. 2, pp. 795-807, Feb. 2020.

[148] T. H. Nguyen, T. K. Nguyen, H. D. Han, and V. D. Nguyen, "Optimal power control and load balancing for uplink cell-free multi-user massive MIMO," IEEE Access, vol. 6, pp. 14 462-14 473, 2018. 
[149] M. Bashar, K. Cumanan, A. G. Burr, M. Debbah, and H. Q. Ngo, "On the uplink max-min SINR of cell-free massive MIMO systems," IEEE Transactions on Wireless Communications, vol. 18, no. 4, pp. 2021-2036, Apr. 2019.

[150] T. C. Mai, H. Q. Ngo, M. Egan, and T. Q. Duong, "Pilot power control for cell-free massive MIMO," IEEE Transactions on Vehicular Technology, vol. 67, no. 11, pp. $11264-11268$, Nov. 2018.

[151] G. Femenias and F. Riera-Palou, "Cell-free millimeter-wave massive MIMO systems with limited fronthaul capacity," IEEE Access, vol. 7, pp. $44596-$ 44 612, 2019.

[152] M. Alonzo, S. Buzzi, A. Zappone, and C. D’Elia, "Energy-efficient power control in cell-free and user-centric massive MIMO at millimeter wave," IEEE Transactions on Green Communications and Networking, vol. 3, no. 3, pp. 651-663, Sep. 2019.

[153] H. T. P. da Silva, R. M. Duarte, M. S. de Alencar, and W. J. L. Queiroz, "Cellfree at millimeter wave frequency simulation using the ray tracing method," in 2020 14th European Conference on Antennas and Propagation (EuCAP), Mar. 2020 , pp. $1-5$.

[154] J. García-Morales, G. Femenias, and F. Riera-Palou, "Energy-efficient accesspoint sleep-mode techniques for cell-free mmWave massive MIMO networks with non-uniform spatial traffic density," IEEE Access, pp. 1-1, 2020.

[155] M. Bashar, K. Cumanan, A. G. Burr, H. Q. Ngo, E. G. Larsson, and P. Xiao, "Energy efficiency of the cell-free massive MIMO uplink with optimal uniform quantization," IEEE Transactions on Green Communications and Networking, vol. 3, no. 4, pp. 971-987, Dec. 2019.

[156] G. Femenias, N. Lassoued, and F. Riera-Palou, "Access point switch on/off strategies for green cell-free massive MIMO networking," IEEE Access, vol. 8, pp. $21788-21803,2020$.

[157] Z. Khodkar and J. Abouei, "Energy efficiency enhancement of cell-free massive multiple-input multiple-output network employing threshold-based beamforming," Transactions on Emerging Telecommunications Technologies, vol. 31, no. 7, p. e4007, 2020.

[158] Q. Huang and A. Burr, "Compute-and-forward in cell-free massive MIMO: Great performance with low backhaul load," in 2017 IEEE International Conference on Communications Workshops (ICC Workshops), May 2017, pp. 601-606. 
[159] J. Zhang, J. Zhang, J. Zheng, S. Jin, and B. Ai, "Expanded compute-andforward for backhaul-limited cell-free massive MIMO," in 2019 IEEE International Conference on Communications Workshops (ICC Workshops), May 2019, pp. 1-6.

[160] P. Parida, H. S. Dhillon, and A. F. Molisch, "Downlink performance analysis of cell-free massive MIMO with finite fronthaul capacity," in 2018 IEEE 88th Vehicular Technology Conference (VTC-Fall), Aug. 2018, pp. 1-6.

[161] M. Bashar, K. Cumanan, A. G. Burr, H. Q. Ngo, M. Debbah, and P. Xiao, "Max-min rate of cell-free massive MIMO uplink with optimal uniform quantization," IEEE Transactions on Communications, vol. 67, no. 10, pp. 6796-6815, Oct. 2019.

[162] X. Yin, J. Dai, and J. Shi, "Performance analysis of cell-free massive MIMO systems under limited feedback," in 2019 IEEE International Conference on Communications Workshops (ICC Workshops), May 2019, pp. 1-6.

[163] G. Femenias and F. Riera-Palou, "Fronthaul-constrained cell-free massive MIMO with low resolution ADCs," IEEE Access, vol. 8, pp. 116 195-116 215, 2020 .

[164] H. Masoumi and M. J. Emadi, "Performance analysis of cell-free massive MIMO system with limited fronthaul capacity and hardware impairments," IEEE Transactions on Wireless Communications, vol. 19, no. 2, pp. 1038-1053, Feb. 2020.

[165] Y. Zhang, M. Zhou, X. Qiao, H. Cao, and L. Yang, "On the performance of cell-free massive MIMO with low-resolution ADCs," IEEE Access, vol. 7, pp. 117 968-117 977, 2019.

[166] X. Hu, C. Zhong, X. Chen, W. Xu, H. Lin, and Z. Zhang, "Cell-free massive MIMO systems with low resolution ADCs," IEEE Transactions on Communications, vol. 67, no. 10, pp. 6844-6857, 2019.

[167] X. Zhao, J. Zhang, J. Zhang, F. Xiong, and B. Ai, "Efficient receiver for cell-free massive MIMO systems with low-resolution ADCs," in 2020 IEEE International Conference on Communications Workshops (ICC Workshops), Jun. 2020, pp. 1-6.

[168] J. Zhang, Y. Wei, E. Björnson, Y. Han, and S. Jin, "Performance analysis and power control of cell-free massive MIMO systems with hardware impairments," IEEE Access, vol. 6, pp. 55 302-55 314, 2018. 
[169] S. Elhoushy and W. Hamouda, "Performance of distributed massive MIMO and small-cell systems under hardware and channel impairments," IEEE Transactions on Vehicular Technology, pp. 1-1, 2020, early access.

[170] J. Zheng, J. Zhang, L. Zhang, X. Zhang, and B. Ai, "Efficient receiver design for uplink cell-free massive MIMO with hardware impairments," IEEE Transactions on Vehicular Technology, vol. 69, no. 4, pp. 4537-4541, Apr. 2020.

[171] Y. Li and G. A. Aruma Baduge, "NOMA-Aided cell-free massive MIMO systems," IEEE Wireless Communications Letters, vol. 7, no. 6, pp. 950-953, Dec. 2018.

[172] S. Kusaladharma, W. P. Zhu, W. Ajib, and G. Amarasuriya, "Achievable rate analysis of NOMA in cell-free massive MIMO: A stochastic geometry approach," in ICC 2019 - 2019 IEEE International Conference on Communications (ICC), May 2019, pp. 1-6.

[173] M. Bashar, K. Cumanan, A. G. Burr, H. Q. Ngo, L. Hanzo, and P. Xiao, "On the performance of cell-free massive MIMO relying on adaptive NOMA/OMA mode-switching," IEEE Transactions on Communications, vol. 68, no. 2, pp. 792-810, Feb. 2020.

[174] F. Rezaei, C. Tellambura, A. A. Tadaion, and A. R. Heidarpour, "Rate analysis of cell-free massive MIMO-NOMA with three linear precoders," IEEE Transactions on Communications, vol. 68, no. 6, pp. 3480-3494, Jun. 2020.

[175] T. K. Nguyen, H. H. Nguyen, and T. D. Hoang, "Max-min QoS power control in generalized cell-free massive MIMO-NOMA with optimal backhaul combining," IEEE Transactions on Vehicular Technology, pp. 1-1, 2020.

[176] C. D’Andrea, A. Zappone, S. Buzzi, and M. Debbah, "Uplink power control in cell-free massive MIMO via deep learning," in 2019 IEEE 8th International Workshop on Computational Advances in Multi-Sensor Adaptive Processing (CAMSAP), Dec. 2019, pp. 554-558.

[177] Y. Jin, J. Zhang, S. Jin, and B. Ai, "Channel estimation for cell-free mmWave massive MIMO through deep learning," IEEE Transactions on Vehicular Technology, vol. 68, no. 10, pp. $10325-10329$, Oct. 2019.

[178] M. Alrabeiah and A. Alkhateeb, "Deep learning for TDD and FDD massive MIMO: Mapping channels in space and frequency," in 2019 53rd Asilomar Conference on Signals, Systems, and Computers, Nov. 2019, pp. 1465-1470. 
[179] N. Athreya, V. Raj, and S. Kalyani, "Beyond 5G: Leveraging cell free TDD massive MIMO using cascaded deep learning," IEEE Wireless Communications Letters, pp. 1-1, 2020.

[180] M. Bashar, A. Akbari, K. Cumanan, H. Q. Ngo, A. G. Burr, P. Xiao, M. Debbah, and J. Kittler, "Exploiting deep learning in limited-fronthaul cell-free massive MIMO uplink," IEEE fournal on Selected Areas in Communications, pp. 1-1, 2020.

[181] H. Q. Ngo, H. Tataria, M. Matthaiou, S. Jin, and E. G. Larsson, "On the performance of cell-free massive MIMO in Ricean fading," in 2018 52nd Asilomar Conference on Signals, Systems, and Computers, Oct. 2018, pp. 980984.

[182] Ȯ. Özdogan, E. Björnson, and J. Zhang, "Performance of cell-free massive MIMO with Rician fading and phase shifts," IEEE Transactions on Wireless Communications, vol. 18, no. 11, pp. 5299-5315, Nov. 2019.

[183] S. Jin, D. Yue, and H. H. Nguyen, "Spectral and energy efficiency in cell-free massive MIMO systems over correlated Rician fading," IEEE Systems fournal, pp. 1-12, 2020.

[184] W. Fan, J. Zhang, E. Björnson, S. Chen, and Z. Zhong, "Performance analysis of cell-free massive MIMO over spatially correlated fading channels," in 2019 IEEE International Conference on Communications (ICC), May 2019, pp. 1-6.

[185] M. Zhou, Y. Zhang, X. Qiao, and L. Yang, "Spatially correlated Rayleigh fading for cell-free massive MIMO systems," IEEE Access, vol. 8, pp. 42 154-42 168, 2020.

[186] J. Qiu, K. Xu, X. Xia, Z. Shen, and W. Xie, "Downlink power optimization for cell-free massive MIMO over spatially correlated Rayleigh fading channels," IEEE Access, vol. 8, pp. $56214-56$ 227, 2020.

[187] T. T. Vu, D. T. Ngo, H. Q. Ngo, and T. Le-Ngoc, "Full-duplex cell-free massive MIMO," in 2019 IEEE International Conference on Communications (ICC), May 2019, pp. 1-6.

[188] D. Wang, M. Wang, P. Zhu, J. Li, J. Wang, and X. You, "Performance of network-assisted full-duplex for cell-free massive MIMO," IEEE Transactions on Communications, vol. 68, no. 3, pp. 1464-1478, Mar. 2020. 
[189] H. V. Nguyen, V. Nguyen, O. A. Dobre, S. K. Sharma, S. Chatzinotas, B. Ottersten, and O. Shin, "On the spectral and energy efficiencies of full-duplex cell-free massive MIMO," IEEE Journal on Selected Areas in Communications, pp. 1-1, 2020, early access.

[190] J. Morte Palacios, O. Raeesi, A. Gokceoglu, and M. Valkama, "Impact of channel non-reciprocity in cell-free massive MIMO," IEEE Wireless Communications Letters, vol. 9, no. 3, pp. 344-348, Mar. 2020.

[191] H. Yan and I. Lu, "Asynchronous reception effects on distributed massive MIMO-OFDM system," IEEE Transactions on Communications, vol. 67, no. 7, pp. 4782-4794, Jul. 2019.

[192] M. Karlsson, E. Björnson, and E. G. Larsson, "Techniques for system information broadcast in cell-free massive MIMO," IEEE Transactions on Communications, vol. 67, no. 1, pp. 244-257, Jan. 2019.

[193] I. Atzeni, B. Gouda, and A. Tölli, "Distributed joint receiver design for uplink cell-free massive MIMO," in 2020 IEEE International Conference on Communications Workshops (ICC Workshops), Jun. 2020, pp. 1-6.

[194] B. Gouda, I. Atzeni, and A. Tölli, "Distributed precoding design for cell-free massive MIMO systems," in 2020 IEEE 21st International Workshop on Signal Processing Advances in Wireless Communications (SPAWC), May 2020, pp. $1-5$.

[195] T. C. Mai, H. Q. Ngo, and T. Q. Duong, "Uplink spectral efficiency of cellfree massive MIMO with multi-antenna users," in 2019 3rd International Conference on Recent Advances in Signal Processing, Telecommunications Computing (SigTelCom), Mar. 2019, pp. 126-129.

[196] --, "Downlink spectral efficiency of cell-free massive MIMO systems with multi-antenna users," IEEE Transactions on Communications, pp. 1-1, 2020, early access.

[197] A. Abdallah and M. M. Mansour, "Efficient angle-domain processing for FDD-based cell-free massive MIMO systems," IEEE Transactions on Communications, vol. 68, no. 4, pp. 2188-2203, Apr. 2020.

[198] A. Papazafeiropoulos, P. Kourtessis, M. D. Renzo, S. Chatzinotas, and J. M. Senior, "Performance analysis of cell-free massive MIMO systems: A stochastic geometry approach," IEEE Transactions on Vehicular Technology, vol. 69, no. 4, pp. 3523-3537, Apr. 2020. 
[199] Z. Chen, E. Björnson, and E. G. Larsson, "Dynamic resource allocation in co-located and cell-free massive MIMO," IEEE Transactions on Green Communications and Networking, vol. 4, no. 1, pp. 209-220, Mar. 2020.

[200] Ö. T. Demir and E. Björnson, "Joint power control and LSFD for wirelesspowered cell-free massive MIMO," arXiv preprint arXiv:2002.09270, 2020. 



\section{Included Papers}





\section{Papers}

The papers associated with this thesis have been removed for copyright reasons. For more details about these see:

http://urn.kb.se/resolve?urn=urn:nbn:se:liu:diva-167218 



\section{Other Recently Published Theses From}

\section{The Division of Communication Systems Department of Electrical Engineering (ISY) Linköping University, Sweden}

Özgecan Özdogan, Analysis of Cellular and Cell-Free Massive MIMO with Rician Fading, Linköping Studies in Science and Technology. Licentiate Thesis, No. 1870, 2020.

Ema Becirovic, On Massive MIMO for Massive Machine-Type Communications, Linköping Studies in Science and Technology. Licentiate Thesis, No. 1868, 2020.

Daniel Verenzuela, Exploring Alternative Massive MIMO Designs: Superimposed Pilots and Mixed-ADCs, Linköping Studies in Science and Technology. Dissertations, No. 2041, 2020.

Trinh Van Chien, Spatial Resource Allocation in Massive MIMO Communication: From Cellular to Cell-Free, Linköping Studies in Science and Technology. Dissertations, No. 2036, 2020.

Amin Ghazanfari, Power Control for Multi-Cell Massive MIMO, Linköping Studies in Science and Technology. Licentiate Thesis, No. 1852, 2019.

Giovanni Interdonato, Signal Processing Aspects of Cell-Free Massive MIMO, Linköping Studies in Science and Technology. Licentiate Thesis, No. 1817, 2018.

Marcus Karlsson, Blind Massive MIMO Base Stations: Downlink Transmission and famming, Linköping Studies in Science and Technology. Dissertations, No. 1950, 2018.

Victor Hei Cheng, Optimizing Massive MIMO: Precoder Design and Power Allocation, Linköping Studies in Science and Technology. Dissertations, No. 1929, 2018.

Christopher Mollén, High-End Performance with Low-End Hardware: Analysis of Massive MIMO Base Station Transceivers, Linköping Studies in Science and Technology. Dissertations, No. 1896, 2017.

Antonios Pitarokoilis, Phase Noise and Wideband Transmission in Massive MIMO, Linköping Studies in Science and Technology. Dissertations, No. 1756, 2016.

Anu Kalidas M. Pillai, Signal Reconstruction Algorithms for Time-Interleaved ADCs, Linköping Studies in Science and Technology. Dissertations, No. 1672, 2015.

Ngo Quoc Hien, Massive MIMO: Fundamentals and System Designs, Linköping Studies in Science and Technology. Dissertations, No. 1642, 2015.

Mirsad Čirkić, Efficient MIMO Detection Methods, Linköping Studies in Science and Technology. Dissertations, No. 1570, 2014.

Reza Moosavi, Improving the Efficiency of Control Signaling in Wireless Multiple Access Systems, Linköping Studies in Science and Technology. Dissertations, No. 1556, 2014. 

